\title{
Contracter à l'heure de la publication en accès ouvert. Une analyse systématique des accords transformants
}

Rapport final

17 décembre 2020

Contrat $\mathrm{n}^{\circ} 206150$

Projet « Socio-Économie de la Publication Scientifique »

Quentin Dufour (Post-doctorant CNRS)

David Pontille (Directeur de recherche CNRS)

Didier Torny (Directeur de recherche CNRS)

Centre de Sociologie de l'Innovation

Mines ParisTech • PSL University

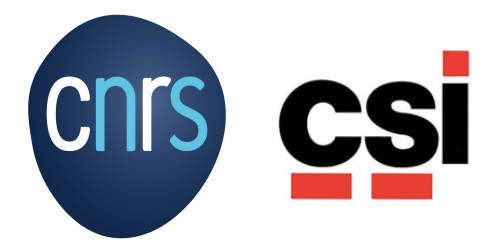

Soutenu par

MI

MINISTÈRE

DE L'ENSEIGNEMENT SUPÉRIEUR,

DE LA RECHERCHE

ET DE L'INNOVATION

Liberté

Égalité 


\section{Sommaire}

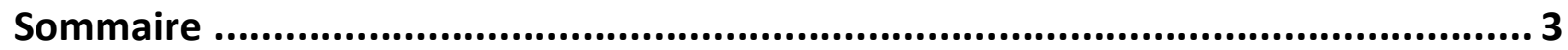

Liste des figures et tableaux .................................................................................... 5

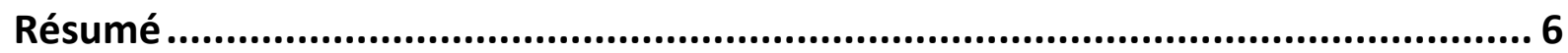

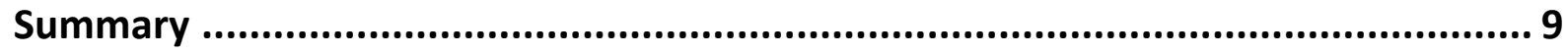

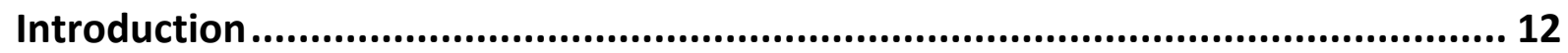

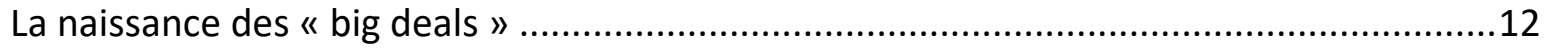

trois modèles parallèles pour une transition vers l'accès ouvert ......................................13

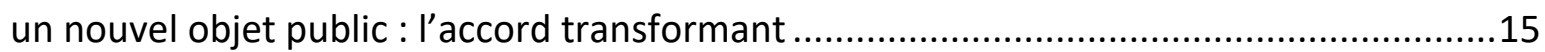

\section{Section 1.}

Méthodologie ...................................................................................................... 18

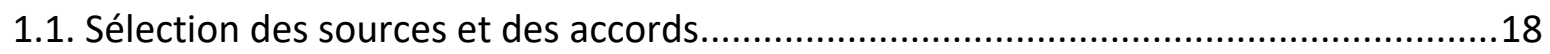

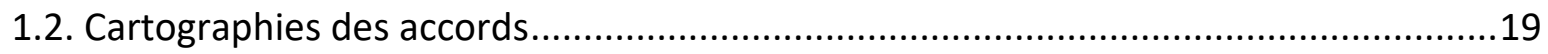

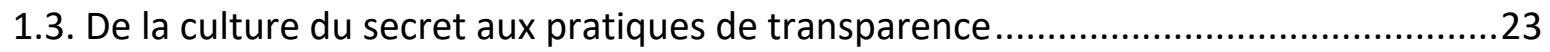

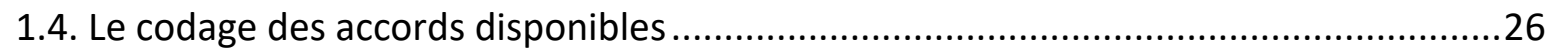

\section{Section 2.}

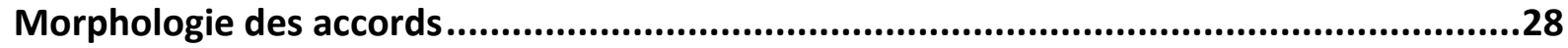

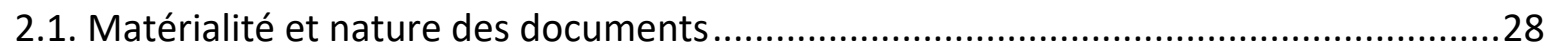

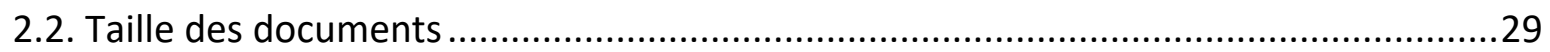

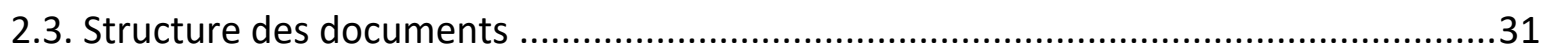

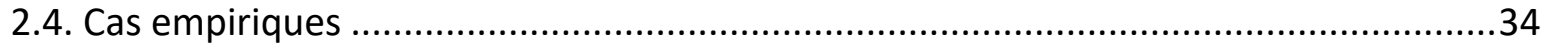

\section{Section 3.}

S'abonner aux revues et publier en accès ouvert...................................................38

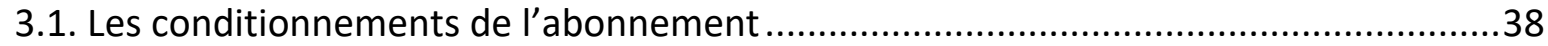

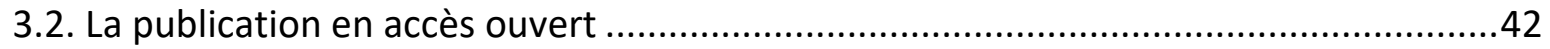

\section{Section 4.}

Deux accords en un : les relations entre abonnement et publication ...................... 50

4.1. Importance relative de l'abonnement et de la publication en accès ouvert................50

4.2. La ventilation des sommes financières ......................................................................52

\section{Section 5.}

Les arrangements financiers de la publication en accès ouvert.............................. 55

5.1. De la prévisibilité à l'incertitude : une catégorisation des arrangements financiers....55 
5.2. Les formules financières de la publication en accès ouvert ....................................59

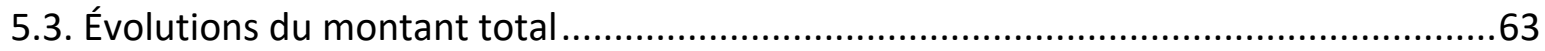

5.4. Répartition des sommes entre les payeurs ........................................................66

\section{Section 6.}

Négocier la transition vers l'accès ouvert ..................................................69 69

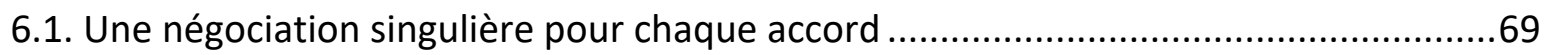

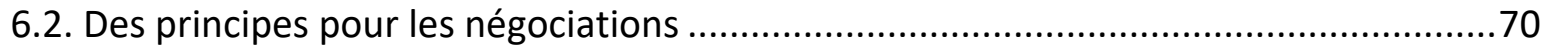

6.3. La publication en accès ouvert comme expérimentation..........................................72

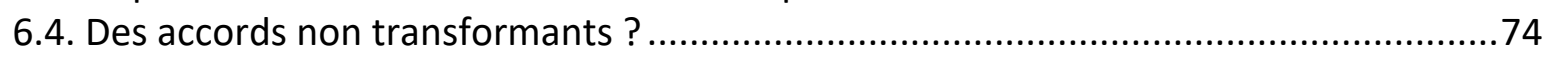

Section 7.

Quels objectifs pour les accords transformants ? ........................................... 78

Annexe : présentations des travaux .................................................... 81 


\section{Liste des figures et tableaux}

\section{Figures}

Figure 1.1 - Répartition des accords par année de début.................................................20

Figure 1.2 - Répartition géographique des accords en Europe...........................................22

Figure 1.3 - Répartition des accords par durée...................................................................23

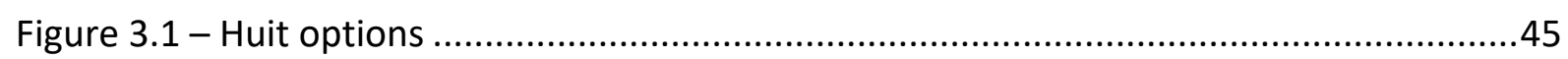

Figure 5.1 - Les coûts de publication du Germany 2020 Springer/DEAL ...............................58

Figure 5.2 - Evolution des montants payés par les institutions de Bibsam à Elsevier ...........65

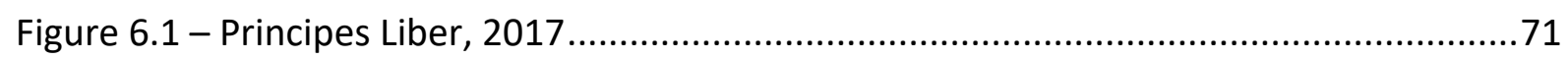

\section{Tableaux}

Tableau 1.1 - Répartition des accords par éditeurs ............................................................21

Tableau 1.2 - Une gamme de pratiques de transparence..................................................24

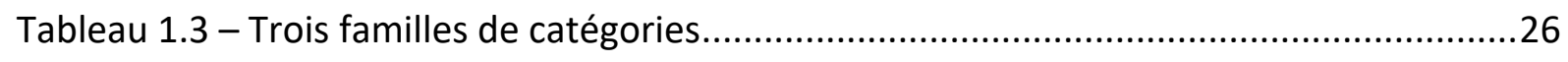

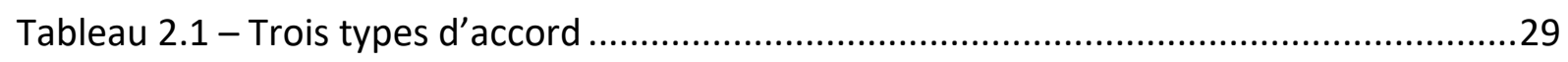

Tableau 2.2 - Taille des documents et part des listes de revues exprimées (en pages) .........30

Tableau 2.3 - Les différentes structures des documents .....................................................34

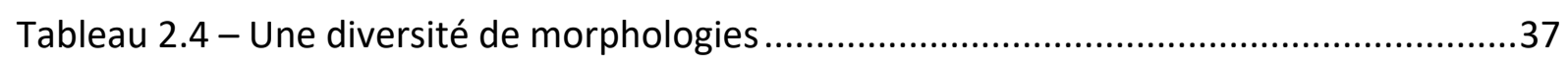

Tableau 3.1 - Distribution différentielle de l'accès aux revues.............................................40

Tableau 3.2 - Exemples empiriques de formules d'accès ouvert........................................49

Tableau 4.1 - Distribution des formes de ventilation financière ...........................................54

Tableau 5.1 - Distribution des arrangements financiers .....................................................56

Tableau 5.2 - Distribution des formules financières .........................................................60

Tableau 5.3 - Exemples de formules financières associées à Somme fixe/volume fixe ..........61

Tableau 5.4 - Exemples de formules financières associées au Corridor ...............................62

Tableau 5.5 - Exemples de formules financières associées au Pay as you publish ................63

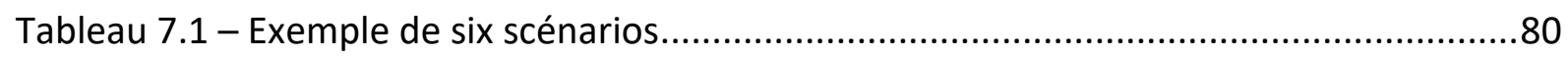


Cette étude porte sur une des innovations contemporaines liées à l'économie de la publication scientifique : les accords dits transformants, un objet relativement circonscrit au sein des relations entre consortiums de bibliothèques et éditeurs scientifiques, et temporellement situé entre 2015 et 2020. Ce type d'accords a pour objectif affiché d'organiser la transition du modèle traditionnel de l'abonnement à des revues (souvent proposées par regroupements thématiques ou collections) vers celui de l'accès ouvert en opérant une réaffectation des budgets qui y sont consacrés.

Notre travail d'analyse sociologique constitue une première étude systématique de cet objet, fondée sur la recension de 197 accords. Le corpus ainsi constitué inclut des accords caractérisés par la coprésence d'une composante d'abonnement et d'une composante de publication en accès ouvert, même minimale (" jetons " de publication offerts, réduction sur les APC...). En conséquence, ont été exclus de l'analyse les accords portant uniquement sur du financement centralisé de publication en accès ouvert, que ce soit avec des éditeurs ne proposant que des revues avec paiement par l'auteur (PLOS, Frontiers, MDPI...) ou des éditeurs dont une partie du catalogue est constitué de revues en accès ouvert. L'accord le plus ancien de notre corpus a été signé en 2010, les plus récents en 2020 - les accords ne commençant qu'en 2021, même annoncés au cours de l'étude, n'ont pas été retenus.

Plusieurs résultats se dégagent de notre analyse. Tout d'abord, on note une grande diversité des acteurs impliqués avec 22 pays et 39 éditeurs, même si certains consortiums (Pays-Bas, Suède, Autriche, Allemagne) et éditeurs (CUP, Elsevier, RSC, Springer) en ont signé beaucoup plus que d'autres. Ensuite, la durée des accords, comprise entre une et six années, révèle une distribution très inégalitaire, avec plus de la moitié des accords (103) signés pour 3 ans, ainsi qu'une faible proportion pour 4 ans ou plus (22 accords). Enfin, en dépit d'appels répétés à la transparence, moins de la moitié des accords (96) ont un texte accessible au moment de cette étude, sans qu'on puisse observer une tendance récente à une plus grande disponibilité. L'analyse montre également des degrés d'ouverture très variables, allant d'une simple information sur le répertoire ESAC en passant par la mise à disposition d'un format annotable jusqu'à l'attribution d'un DOI et d'une licence de réutilisation (CC-BY), en incluant le détail des sommes monétaires.

Parmi les 96 accords disponibles, dont 47 signés en 2020, 62 ont fait l'objet d'une analyse en profondeur. C'est à notre connaissance la première analyse à cette échelle, sur un type de matériel non seulement inédit, mais qui était auparavant soumis à des clauses de confidentialité. Fondée sur une lecture minutieuse, l'étude décrit de manière fine leurs 
propriétés, depuis la matérialité du document jusqu'aux formules financières, en passant par leur morphologie et l'ensemble des droits et devoirs des parties. Les contenus des accords sont donc analysés comme une collection dont nous cherchons à déterminer les points communs et les variations, à travers des codages explicites sur certaines de leurs caractéristiques. L'étude pointe également des incertitudes, et notamment leur caractère " transitionnel ", qui demeure fortement discuté.

D'un point de vue morphologique, les accords montrent une grande diversité en matière de taille (de 7 à 488 pages) et de structure. Néanmoins, par définition, ils articulent tous deux objets essentiels : d'une part, les conditions de réalisation d'une lecture d'articles de revues, sous forme d'abonnement, mêlant des préoccupations d'accès et de sécurité ; d'autre part, les modalités de publication en accès ouvert, articulant la gestion d'un nouveau type de workflow à toute une série d'options possibles. Parmi ces options, mentionnons notamment le périmètre des revues considérées (hybrides et/ou accès ouvert), les licences disponibles, le degré d'obligation de cette publication, les auteurs éligibles ou le volume d'articles publiables.

L'un des résultats les plus importants de cette analyse approfondie est la mise au jour d'un découplage presque complet, au sein même des accords, entre l'objet abonnement et l'objet publication. Bien entendu, l'abonnement est systématiquement configuré dans un monde fermé, soumis à paiement qui déclenche des séries d'identification des circulations légitimes tant du contenu informationnel que des usagers. II insiste notamment sur les interdictions de réutilisation ou même de copie des articles scientifiques. À l'opposé, la publication en accès ouvert est attachée à un monde régi par l'accès gratuit au contenu, ce qui induit des préoccupations de gestion du workflow et des modalités d'accessibilité. De plus, les différents éléments constitutifs de ces objets contractuels ne sont pas couplés : d'un côté, les lecteurs sont constitués de l'ensemble des membres des institutions abonnées, de l'autre, seuls les auteurs correspondants ("corresponding authors") sont concernés ; les listes de revues accessibles à la lecture et celles réservées à la publication en accès ouvert sont le plus souvent distinctes; les workflows ont des objectifs et des organisations matérielles totalement différentes, etc. L'articulation entre les deux objets contractuels relève uniquement d'une formule de distribution financière qui, outre des combinaisons particulières entre l'un et l'autre, permet d'attribuer des étiquettes distinctes aux accords (offset agreement, publish \& read, read \& publish, read \& free articles, read \& discount).

Au-delà de cette distribution, l'étude des arrangements financiers montre une gamme de dispositions allant d'une prévisibilité budgétaire totale, donc identique aux accords d'abonnement antérieurs, à une incertitude sur le volume de publication ou sur le montant définitif des sommes échangées. Les modalités concrètes de calcul des montants associés à la publication en accès ouvert sont relativement variées. S'il existe effectivement des formules récurrentes (volume d'articles multiplié par un prix individuel, reprise de la moyenne des 
sommes totales d'APC des années précédentes...), le calcul des sommes en jeu est toujours le résultat d'une négociation singulière entre un consortium et un éditeur scientifique, et aboutit parfois à des formules originales et complexes. À ce titre, l'espace des possibles en matière de formules financières n'est jamais totalement clos. Par ailleurs, la volonté des consortiums d'opérer une " transformation » de leurs accords vers la publication à coût constant renvoie à des définitions diversifiées du " coût " (inclusion ou non des dépenses d'APC préexistantes) et de la constance (admission ou pas d'une «inflation » à 2 ou 3\%). De plus, nous n'avons observé aucune disposition contractuelle permettant d'anticiper les sommes en jeu au-delà de l'horizon temporel de l'accord courant.

La grande diversité des accords provient d'une part des conditions initiales des relations entre consortiums et éditeurs scientifiques - les sommes dépensées en abonnement étant le point de départ des nouveaux accords -, d'autre part des objectifs de chaque partie. Même si cette étude excluait volontairement les négociations, les accords portent des traces de ces objectifs. Ainsi, de nombreux accords sont de nature explicitement expérimentale, quand certains visent un contrôle budgétaire strict, ou d'autres ambitionnent, dans la période plus récente, la publication du plus grand nombre possible d'articles en accès ouvert. C'est dans ce dernier cas qu'on touche à l'ambiguïté des attentes générales sur les accords transformants. En effet, pour les consortiums, la dimension " transformante " consiste essentiellement à transférer les sommes traditionnellement allouées à l'abonnement vers la publication en accès ouvert. Mais l'objectif n'est jamais de transformer le modèle économique des revues, c'est-à-dire de faire basculer des revues sous abonnement ou hybrides en revues entièrement en accès ouvert. D'ailleurs, aucune clause ne vise une telle fin - à l'exception du modèle d'accord proposé par l'éditeur ACM. Du côté des éditeurs, et notamment de Springer, le caractère cumulatif des accords nationaux passés vise à projeter un monde de la publication où l'accès ouvert devient de fait quantitativement très dominant, sans pour autant modifier de manière pérenne le modèle économique de leurs revues.

Notre étude montre que les accords transformants actuels ne permettent pas d'assurer de manière durable une transition de l'économie de la publication vers l'accès ouvert, dans la mesure où ils n'offrent pas de garantie sur le contrôle des dépenses ni sur la pérennité de l'ouverture des contenus. L'avenir des relations entre consortium et éditeur demeure largement indéterminé. 


\section{Summary}

This study focuses on one of the contemporary innovations linked to the economy of academic publishing: the so-called transformative agreements, a relatively circumscribed object within the relations between library consortia and academic publishers, and temporally situated between 2015 and 2020. The stated objective of this type of agreement is to organise the transition from the traditional model of subscription to journals (often proposed by thematic groupings or collections) to that of open access by reallocating the budgets devoted to it.

Our sociological analysis work constitutes a first systematic study of this object, based on a review of 197 agreements. The corpus thus constituted includes agreements characterised by the co-presence of a subscription component and an open access publication component, even minimal (publication "tokens" offered, reduction on APCs, etc.). As a result, agreements that only concern centralised funding for open access publishing were excluded from the analysis, whether with publishers that only offer journals with payment by the author (PLOS, Frontiers, MDPI, etc.) or publishers whose catalogue includes open access journals. The oldest agreement in our corpus was signed in 2010, the most recent ones in 2020 - agreements starting only in 2021, even announced during the study, were not retained.

Several results emerge from our analysis. First of all, there is a great diversity of actors involved with 22 countries and 39 publishers, even if some consortia (Netherlands, Sweden, Austria, Germany) and publishers (CUP, Elsevier, RSC, Springer) signed many more than others. Secondly, the duration of the agreements, ranging from one to six years, reveals a very unequal distribution, with more than half of the agreements (103) signed for 3 years, and a small proportion for 4 years or more (22 agreements). Finally, despite repeated calls for transparency, less than half of the agreements (96) have an accessible text at the time of this study, with no recent trend towards greater availability. The analysis also shows widely varying degrees of openness, ranging from simple information on the ESAC directory through the provision of an open format to the allocation of a DOI and a reuse licence (CC-BY), including details of monetary amounts.

Of the 96 agreements available, 47 of which were signed in 2020, 62 have been analysed in depth. To our knowledge, this is the first analysis on this scale, on a type of material that was not only unpublished, but which was previously subject to confidentiality clauses. Based on a careful reading, the study describes in detail their properties, from the materiality of the document to the financial formulas, including their morphology and all the rights and duties of the parties. We therefore analysed the content of the agreements as a collection, looking for commonalities and variations through an explicit coding of their 
characteristics. The study also points out some uncertainties, in particular their "transitional" character, which remains strongly debated.

From a morphological point of view, the agreements show a great diversity in size (from 7 to 488 pages) and structure. Nevertheless, by definition, they both articulate two essential objects: on the one hand, the conditions for carrying out a reading of journal articles, in the form of a subscription, combining concerns of access and security; on the other hand, the modalities of open access publication, articulating the management of a new type of workflow with a whole series of possible options. These options include the scope of the journals considered (hybrid and/or open access), the licences available, the degree of obligation to publish, the eligible authors or the volume of publishable articles.

One of the most important results of this in-depth analysis is the discovery of an almost complete decoupling, within the agreements themselves, between the subscription object and the publication object. Of course, subscription is systematically configured in a closed world, subject to payment, which triggers series of identification of legitimate circulations of both information content and users. In particular, it insists on prohibitions on the reuse or even copying of academic articles. On the other hand, open access publishing is attached to a world governed by free access to content, which leads to concerns about workflow management and accessibility modalities. Moreover, the different elements that make up these contractual objects are not interconnected: on one side, the readers are all members of the subscribing institutions, on the other, only the corresponding authors are concerned; the lists of journals accessible to the reader and those reserved for open access publication are usually distinct; the workflows have totally different objectives and material organisations, etc. The articulation between the two contractual objects is solely a matter of a financial distribution formula which, in addition to particular combinations between one and the other, makes it possible to assign distinct labels to the agreements (offset agreement, publish \& read, read \& publish, read \& free articles, read \& discount).

Beyond this distribution, the study of financial arrangements shows a range of provisions from total budgetary predictability, thus identical to previous subscription agreements, to uncertainty about the volume of publication or the final amount of the sums exchanged. The concrete methods of calculating the amounts associated with open access publication are relatively varied. While there are indeed recurrent formulas (volume of articles multiplied by an individual price, taking the average of the total APC sums from previous years, etc.), the calculation of the sums involved is always the result of a singular negotiation between a consortium and an academic publisher, and sometimes leads to original and complex formulas. As such, the space of possibilities in terms of financial formulas is never completely closed. Furthermore, the willingness of consortia to "transform" their agreements towards publication at constant cost refers to diversified definitions of "cost" (whether or not to include pre-existing APC expenditure) and stability (whether or not to allow for "inflation" 
of 2 or $3 \%$ ). Furthermore, we have not observed any contractual provisions that would allow us to anticipate the sums involved beyond the time horizon of the current agreement.

The great diversity of the agreements stems, on the one hand, from the initial conditions of the relations between consortia and academic publishers - the sums spent on subscriptions being the starting point for the new agreements - and, on the other hand, from the objectives of each party. Even if this study deliberately excluded negotiations, the agreements bear traces of these objectives. Thus, many agreements are explicitly experimental in nature, while some aim at strict budgetary control, or others aim, in the more recent period, at publishing as many articles as possible in open access. It is in the latter case that we reach the ambiguity of general expectations about transformative agreements. Indeed, for consortia, the transforming dimension essentially consists in transferring the sums traditionally allocated to subscription to open access publication. But the goal is never to transform the economic model of journals, i.e. to shift from subscription or hybrid journals to fully open access journals. Moreover, no clause aims at such an end - with the exception of the model agreement proposed by the publisher ACM. On the side of the publishers, and in particular Springer, the cumulative nature of the national agreements concluded is aimed at projecting a world of publishing in which open access becomes de facto quantitatively very dominant, without changing the economic model of their journals.

Our study shows that the current transformative agreements do not provide for a sustainable transition of the publishing economy towards open access, as they do not guarantee control of expenditure or the sustainability of open content. The future of the relationship between consortia and publishers remains largely undetermined. 


\section{Introduction}

Ce travail d'analyse sociologique se focalise sur un objet relativement circonscrit, temporellement situé entre 2015 et 2020, dans les relations entre consortiums de bibliothèques et éditeurs scientifiques. II s'agit des accords dits transformant, signés entre les éditeurs scientifiques et les consortiums de bibliothèques universitaires, dont l'objectif affiché est d'organiser la transition du modèle traditionnel de l'abonnement vers celui de l'accès ouvert en réaffectant les budgets qui y étaient consacrés. Dans cette introduction, avant de rappeler les objectifs de l'étude, nous retraçons de manière très synthétique les éléments qui ont conduit à l'émergence de ces accords et le paysage dans lequel ils se situent ${ }^{1}$.

\section{La naissance des « Big Deals »}

À la fin des années 1990 trois événements majeurs ont eu cours dans les relations entre bibliothèques et éditeurs scientifiques. Premièrement, dans une période relativement courte entre la naissance du world wide web et la fin du siècle, les plus gros éditeurs ont mis en ligne non seulement l'ensemble de leur catalogue contemporain, mais également une partie de leurs archives. Deuxièmement, des éditeurs ont proposé des formules d'accès, non plus au titre à titre, mais à l'ensemble de leurs revues. Troisièmement, pour que cette offre soit intéressante, ils ont favorisé l'émergence de consortiums de bibliothèque qui, par addition de leurs besoins singuliers, pouvaient constituer des clients intéressés par cette nouvelle offre pléthorique. La combinaison de ces trois événements ont fait naître le " big deal » comme forme d'accord économique, et le consortium comme partenaire principal des éditeurs en lieu et place des bibliothèques d'une institution de l'enseignement supérieur et de la recherche, particulièrement en Europe.

Les années 2000 ont vu l'émergence d'une offre concurrente à celle des éditeurs traditionnels : les revues en accès ouvert sont sorties de leurs niches des années 1990 pour se généraliser dans toutes les disciplines, suivant des modèles économiques diversifiés, conformément à la prévision de la Budapest Open Access Initiative (BOAI). Se faisaient ainsi face deux mondes très différents : d'un côté des revues en accès payant, sous identification, rassemblées sur de grands portails d'éditeurs, de l'autre des revues chez de multiples éditeurs,

\footnotetext{
${ }^{1}$ Pour une histoire beaucoup plus détaillée, voir Borrego, A. Anglada L. and Abadal E., (2020) « Transformative Agreements: Do They Pave The Way To Open Access? ", Learned Publishing, [Online first: 03 December 2020] https://doi.org/10.1002/leap.1347
} 
dont les textes étaient librement accessibles au lecteur, et donc "gratuites " pour les bibliothèques. Entre ces deux mondes, il existait certes quelques points de passage, dont les revues à barrière mobile ${ }^{2}$ et les revues hybrides ${ }^{3}$, et certaines plateformes de part et d'autre pouvant relever du même propriétaire, mais des logiques de financement et des modèles économiques très différents sont à l'œuvre.

Les revues en accès libre ont très tôt sollicité les bibliothèques afin de recevoir leur soutien, qu'elles soient fondées sur un modèle " diamant ", gratuit pour les auteurs ou sur un modèle de paiement de "frais de publication » par les auteurs (Article Processing Charge APC). Ainsi, BMC, pionnier de l'APC, pratiquait des accords institutionnels dès 2004 afin de prendre en charge les frais d'APC à la place des auteurs. Mais du côté des éditeurs traditionnels, hors des revues hybrides particulièrement développées par Springer à travers son programme Open Choice, on n'observait guère de velléités effectives de mise en œuvre de l'accès ouvert ni de recherche de développement de nouvelles relations avec les bibliothèques au-delà des "Big Deals " 4 .

\section{Trois modèles parallèles pour une transition vers l'accès ouvert}

C'est bien du côté des bibliothèques et des universités que sont véritablement nées les initiatives pour favoriser le basculement des revues sous abonnement vers l'accès ouvert à partir de la fin des années 2000 , les promesses du début de cette même décennie n'ayant pas conduit à une transformation réellement tangible.

Premièrement, c'est dans une communauté disciplinaire singulière, la physique des hautes énergies, qu'a émergé le projet SCOAP3 (Sponsoring Consortium for Open Access Publishing in Particle Physics). Cette discipline est caractérisée par le gigantisme des infrastructures, le très grand nombre d'auteurs par article, une organisation internationale du financement et du travail de recherche, l'usage massif d'un serveur de preprint, ArXiv, et le tout petit nombre de revues et en conséquence d'éditeurs publiant leurs travaux. En 2007, une première présentation du projet pose les bases d'une transition rapide en désignant le CERN comme l'organisation hôte susceptible de gérer le paiement d'APC aux éditeurs avant une répartition auprès des 3000 partenaires du consortium. Le montant des APC est fixé par

\footnotetext{
${ }^{2}$ Une revue à barrière mobile est une revue pour laquelle les contenus sont d'abord uniquement accessibles sous abonnement, avant d'être accessibles à tous après un certain laps de temps (six mois, un an, deux ans...). Mais le contenu n'est pas pour autant sous une licence de type Creative Commons.

${ }^{3}$ Les revues dites « hybrides » proposent certains articles uniquement sous abonnement et d'autres articles en accès ouvert. L'ouverture des articles dans les revues hybrides n'est généralement pas automatique : elle suppose une demande de l'auteur ou de son institution - sauf dans le cas de certains accords transformants qui rendent l'ouverture obligatoire - et implique une transaction financière entre auteurs et éditeur.

${ }^{4}$ À quelques exceptions près, dont le programme « Gold for Gold » de la Royal Society of Chemistry à partir de 2013.
} 
appel d'offres et les bibliothèques ne s'engagent qu'à hauteur de leur budget d'abonnement, les éditeurs baissant leur prix au fur et à mesure de l'encaissement des APC. Mis réellement en place en 2014, le modèle a depuis été étendu à des revues ne publiant que partiellement de la physique des hautes énergies, ainsi qu'à de nouveaux membres dans le consortium. Aujourd'hui plus de 37000 articles ont été publiés en accès ouvert par ce mécanisme mondial de financement.

Deuxièmement, c'est une politique publique nationale, celle du Royaume-Uni mise en place après le Finch Report en 2012, qui a tenté d'accélérer la transition vers l'accès ouvert par un financement collectif. II se focalise d'abord sur des "blocks grants " ${ }^{5}$ d'APC qui apportent un financement supplémentaire aux éditeurs traditionnels, avant qu'en 2014 le ministre Willets reconnaisse l'importance du JISC, le grand consortium britannique, dans la coordination des relations avec les éditeurs, notamment dans la signature des "offsetting agreements " liant dépenses d'abonnement et paiement pour la « libération » des articles. Cette logique de compensation n'a toutefois pas à être entière et certains éditeurs, dont Elsevier, ne signeront pas de tels accords. En revanche, le consortium anglais JISC a conclu un grand nombre d'accords de ce type à partir de 2015. Les principes guidant ce type d'accords sont définis début 2016 dans un document rendu public ${ }^{6}$, avec une gamme d'options articulant en pratique abonnement à des revues et publication en accès ouvert dans tout ou partie de cette liste de revues.

Troisièmement, c'est une politique institutionnelle d'un établissement de recherche, le Max Planck, qui a défini un objectif ambitieux : la fin des abonnements et leur remplacement par une publication ouverte généralisée. Pour ce faire, il suffirait de réaffecter l'ensemble des budgets d'abonnement actuels au paiement des publications. Exposée dans un « rapport blanc » de la Max Planck Digital Library (MDPL) en 20157, cette vision gagna rapidement des soutiens à travers l'initiative OA2020, qui affirmait la nécessité d'une " transformation rapide » des budgets d'abonnement vers un financement par APC. Un autre projet de la MDPL, Efficiency and Standards Articles Charges (ESAC), qui visait initialement à produire des standards de données pour les APC, fut utilisé pour développer des réseaux autour des nouveaux offsetting agreements comme l'un des objets importants des APC. La volonté de la MDPL et d'autres institutions allemandes, soutenues par le grand financeur

\footnotetext{
${ }^{5}$ Les blocks grants sont des fonds réguliers dispensés par les agences publiques de financement de la recherche, dont l'objectif est de financer des projets spécifiques, comme le paiement des APC dans le cadre des politiques d'accès ouvert au Royaume-Uni.

${ }^{6}$ https://www.jisc-collections.ac.uk/Global/News\%20files\%20and\%20docs/Principles-for-offsetagreements.pdf

${ }^{7}$ Schimmer, K. Geschuhn and A. Vogler, Disrupting the subscription journals 'business model for the necessary large-scale transformation to open access. A Max Planck Digital Library Open Access Policy White Paper, München, MPDL 2015, http://dx.doi.org/10.17617/1.3
} 
fédéral Deutsche Forschungsgemeinschaft (DFG), de rendre transparent le marché des APC à travers le projet INTACT ${ }^{8}$, a ainsi produit un effet de bord en organisant des discussions transnationales sur les nouveaux accords avec les éditeurs scientifiques, parfois alors qualifiés d' «OA big deals». Ainsi, c'est sans doute en janvier 2018, lors d'un workshop toujours organisé par ESAC, que la notion de " transformative deal » a été popularisée pour la première fois. Enfin, en octobre 2018, le site ESAC est relancé, avec la volonté explicite d'en faire un lieu de partage d'informations à destination des bibliothèques et consortiums. Loin d'être simplement un lieu destiné à recenser des guidelines, ESAC encourage le dépôt d'un maximum d'informations sur les accords en cours afin de développer une "culture du partage ", là où la culture professionnelle du secret avait longtemps été dominante ${ }^{9}$.

\section{Un nouvel objet public : l'accord transformant}

Parmi les infrastructures de l'accès ouvert, les catalogues jouent un rôle très important : DOAJ, DOAB, SHERPA-ROMEO, ROAR, ROAD sont autant de sigles devenus familiers pour des dispositifs pérennes de catalogage, rassemblant des objets partageant certaines propriétés. II n'est guère étonnant qu'ESAC ait conçu de construire un tel répertoire pour ces nouveaux accords. Dénommé "Transformative Agreements Registry», il participe à la diffusion de la notion d'accord transformant. Le répertoire est alimenté, suivant une logique collaborative, par les consortiums eux-mêmes qui doivent remplir un formulaire avec une vingtaine de questions, certaines d'identification des parties, d'autres à choix multiples, et enfin quelquesunes en texte libre. Ces informations sont soit partagées sur le site, soit conservées par ESAC, la seule mention de l'accord étant alors inscrite dans le répertoire. C'est ainsi qu'ont été rapidement référencés des dizaines d'accords, pour de nombreux pays et éditeurs. L'accord transformant est ainsi devenu en 2019 un objet de discussion publique, d'échanges d'informations systématiques entre consortiums... et de recherche pour notre équipe.

En effet, le développement rapide de cette nouvelle forme d'accord intéresse le ministère de l'Enseignement supérieur, de la Recherche et de l'Innovation et le Comité pour la science ouverte et, parce qu'elle constitue une "nouvelle voie " pour parvenir à un accès ouvert généralisé. Dans un contexte français marqué un niveau élevé d'usage d'archives ouvertes à travers HAL et par l'existence d'un consortium national bien installé, la question du refus ou de l'acceptation des propositions d'accords transformants de la part des éditeurs doit pouvoir être instruite en prenant appui sur les expériences étrangères, et en particulier européennes. En complément des échanges professionnels désormais très nombreux sur les

\footnotetext{
8 https://intact-project.org/

${ }^{9}$ https://esac-initiative.org/happy-open-access-week-lets-develop-a-culture-of-sharing-esac-initiativebecomes-an-information-sharing-hub-for-oa-negotiators/
} 
" contrats", la mise à disposition du texte complet ou quasi-complet des accords constitue une occasion idéale de discuter largement de l'intérêt de ces accords transformants ou de certains d'entre eux pour les acteurs français.

La publicité donnée aux accords par ESAC se double en effet de très fortes incitations à rendre public l'accord lui-même, et ses termes de négociation. L'une des questions du formulaire porte directement sur cette question de la publicité de l'accord, alors que différentes requêtes fondées sur des lois de liberté de l'information ou de la transparence des dépenses publiques ont amené à la diffusion de copies des accords sur les sites des consortiums ou de nouveaux sites dédiés. Ainsi, aux Pays-Bas, des demandes d'accès aux documents contractuels ont premièrement abouti à la publication par la VSNU de huit accords en 2017. Au printemps 2019, le site consacré à l'accès ouvert commença à inclure systématiquement un lien vers le texte des accords, là où il renvoyait auparavant au site de l'éditeur mettant plutôt en œuvre le protocole de publication à destination des auteurs ${ }^{10}$. II faut ajouter que les accords, comme dans la plupart des pays, sont rédigés ou traduits en anglais.

C'est sur la base de cette publicité que nous avons pu lancer notre étude avec des objectifs clairement définis : premièrement, nous souhaitons décrire de manière fine les propriétés mêmes des accords, depuis la matérialité du document jusqu'aux formules financières, en passant par leur morphologie et l'ensemble des droits et devoirs des parties qu'ils définissent. Deuxièmement, nous traitons ces documents comme une collection dont nous cherchons à déterminer les points communs et les variations, à travers des codages explicites sur certaines de leurs caractéristiques. Troisièmement, nous analysons les mondes de l'accès ouvert qu'ils engendrent, à partir d'une matrice fondée sur une tout autre vision, celle de l'accès fermé et de l'abonnement. Quatrièmement, en conclusion, nous soulignerons les incertitudes qu'ils portent: d'une part, ils ne permettent pas de saisir l'ensemble des accords entre auteurs, propriétaires de revue, bibliothèques et éditeurs, d'autre part le caractère " transitionnel " de ces accords est fortement discuté en raison de leur absence d’irréversibilité.

C'est bien le double caractère de nouveauté qui motive cette recherche, puisque d'une part les accords antérieurs de " pur abonnement " n'étaient jamais publics, d'autre part leur forme d'association entre abonnement et publication en accès ouvert est inédite. Pour rassembler ce matériel original, au-delà de l'exploitation de la base ESAC et des liens directs renvoyant vers d'autres sites, nous avons exploré les sites web, plus ou moins visibles, des consortiums. Nous avons donc, d'une part, collecté toutes les annonces effectives d'accords incluant de l'accès ouvert et, d'autre part, accumulé tous les accords eux-mêmes s'ils étaient

${ }^{10}$ https://www.openaccess.nl/en/in-the-netherlands/publisher-deals\#row 
disponibles. L'ensemble de ces éléments, ainsi que nos codages, constitue une base qui est remise au ministère de l'Enseignement supérieur, de la Recherche et de l'Innovation avec ce rapport final d'étude. La liste des principales sources est reproduite ci-après dans le tableau 1. Néanmoins, nous savons qu'il existait d'autres accords qui ne sont pas répertoriés (ni dans ESAC ni dans notre corpus). C'était le cas par exemple les accords polonais, sur lesquels nous n'avions que très peu d'informations, avant que des échanges avec ESAC aient sans doute conduit à leur inclusion, mais d'autres demeurent sans doute relativement invisibles.

Au cours du rapport, nous développerons une nomenclature destinée à caractériser certains éléments financiers de ces accords, mais nous précisons dès cette introduction nos choix lexicaux pour désigner ces documents désormais publics. En français, bien que les termes d'accord et de contrat soient relativement synonymes, nous avons choisi le premier, plus englobant, notamment parce que les accords peuvent contenir des éléments de nature extra-contractuelle (description de processus, affirmations générales sur les objectifs des parties). Nous avons choisi de ne pas qualifier l'ensemble de la collection d'accords, ni de compensation (offset) ni transformants (transformative), ni de publication (publish \& read), en raison de leur grande variété et de l'impossibilité de les classer tous sous une seule catégorie sans risquer d'aplatir leurs différences. Le critère minimal est simplement l'association abonnement/publication en accès ouvert dans un même accord. Précisons deux cas limites qui constituent les marges de notre objet d'étude, et qui ne font à ce titre pas partie du corpus retenu : d'une part des accords dits de " pure publish » signés avec des éditeurs de revues en accès ouvert qu'ils soient nouveaux (PLOS, Frontiers, MDPI, etc.) ou historiques (Springer), et donc par définition ne contiennent pas de dispositions liés à un abonnement, d'autre part les absences de renouvellement d'accords (« no deal »), lorsqu'ils font l'objet d'une grande publicité de la part de consortiums.

Enfin, par anticipation de la diversité des publics susceptibles de lire ce rapport et pour faciliter son appropriation, nous avons limité la discussion proprement académique des différents objets abordés et, en conséquence, les références aux publications scientifiques et à la littérature grise aujourd'hui en forte expansion sur le sujet. Ces éléments seront présents dans les publications scientifiques tirées de notre travail, le financement du ministère étant bien sûr systématiquement mentionné. 


\section{Section 1.}

\section{Méthodologie}

Dans cette section, nous traiterons des manières dont nous avons réuni un corpus d'accords conformes à l'objet de l'étude, leur distribution spatiale et temporelle, ainsi que les modèles progressifs de transparence qui se sont mis en place les cinq dernières années.

\subsection{Sélection des sources et des accords}

Le simple objectif d'origine consistant à recenser les accords transformants nous a immédiatement confronté à un problème de définition et de périmètre: sous quelles conditions la "transformation " conduirait-elle à l'éligibilité dans notre corpus ? Faut-il inclure des accords qui se focalisent majoritairement ou presque exclusivement sur de l'abonnement ? Comment prendre en compte les échecs de négociation ?

Pour répondre à ces questions, par inférences successives et lecture des premiers matériaux rassemblés, nous avons choisi une voie très inclusive. Ainsi, nous avons abandonné toute qualification a priori des "contrats" quant à leur potentiel de changement de l'économie politique de la publication, pour ne retenir que la notion d'accord matérialisée par un texte signé par plusieurs parties. Nous avons également choisi d'enregistrer les " no deal » faisant l'objet d'une communication par les consortiums, et parfois les éditeurs eux-mêmes, parce qu'ils constituent des événements et surtout parce qu'ils explicitent en creux ce qui serait un "bon accord". Enfin, nous avons choisi de retenir dans un premier temps toute référence à l'accès ouvert dans les accords, même sous une forme minimale, comme la possibilité d'avoir des offres sous forme de "jetons " pour la publication d'articles en accès ouvert.

Une fois ces critères fixés, nous devions nous assurer de capturer, si ce n'est l'exhaustivité des accords entrant dans la définition, du moins le plus grand nombre possible. Alors que le site ESAC était un point de départ indispensable, il s'est vite révélé limité dans son extension, directement indexé sur le volontariat des consortiums. On y trouve certes des informations importantes - et parfois non rappelées dans les accords, comme l'évolution du coût par rapport aux abonnements précédents - mais uniquement pour les consortiums les plus actifs ou les plus partageurs, soit environ les trois quarts des accords sur notre corpus final. 
Aussi nous avons exploré de manière systématique les sites web des consortiums où l'information sur les accords est valorisée de manière très variable : Deal; openaccess.nl; Bibsam ; openaccess.no; University of Vienna (une université et non un consortium) ; Finelib; JISC; EISZ ont constitué des sources secondaires cruciales. Nous avons pu observer que certains accords existaient sur ces sites, mais n'étaient pas signalés sur ESAC, sans que l'on puisse déterminer une logique particulière à cette absence de croisement. Ces sites ont fourni environ un quart des accords inclus.

Nous avons ensuite complété nos recherches en passant par les sites des éditeurs, qui ne contiennent jamais les textes des accords, mais plutôt des vade-mecum destinés aux auteurs relevant des institutions les ayant signés. Ces sites ont donc servi d'indices pour renseigner l'existence d'accords non encore publics ou non mentionné du côté des consortiums. De plus, nous avons recueilli de manière systématique les communiqués de presse généralement coproduits par les parties, comme matériel supplémentaire susceptible d'alimenter nos analyses au-delà de ce rapport.

Enfin, nous avons documenté les " no deals », outre les informations présentes sur les sites des consortiums, par l'exploration de la base systématique mise en place par SPARC pour I'abandon des "Big Deals». Cette recherche nous a permis de réaliser que les raisons de ces arrêts sont loin d'être majoritairement liés aux questions d'accès ouvert, mais plutôt aux questions financières dérivant de l'interminable « serial crisis".

\subsection{Cartographies des accords}

La collecte des accords s'est donc appuyée sur les sources décrites ci-dessus, avec des passages successifs au cours de l'étude, puisque plusieurs mois peuvent s'écouler entre l'annonce d'un accord ou du principe d'un accord (" memorandum of understanding "), sa signature effective, et le partage public, partiel ou total, de l'accord sur une url stable. Au moment de la remise de ce rapport, nous avons listé 197 accords. Parmi eux, 96 accords étaient disponibles tandis que 101 accords ne l'étaient pas ou pas encore. À ces 197 accords recensés s'ajoutent 12 annonces d'échec des négociations, de « no deal » entre un consortium et un éditeur. Pour chaque élément entrant dans notre base de données, nous avons construit un identifiant unique qui reprend le type d'objet (TA pour un accord transformant, ND pour un No Deal, PP pour un Pure Publish), les initiales du pays et du consortium, les initiales de l'éditeur, l'année de signature, et le numéro de liste une fois la base stabilisée. Par exemple, l'accord autrichien signé par KËMO avec l'éditeur ACS en 2020 est codé : «TA_AUS/KEMO_ACS_2020_\#3".

Nous décrivons ici la totalité du corpus, l'accord le plus ancien datant de 2010 et les plus récents ne se mettant en place qu'au début de l'année 2020, et non le corpus courant. Néanmoins, la morphologie temporelle montre le caractère exponentiel de la signature des 
accords avec une accélération importante en 2019 et 2020, si on considère que le mouvement actuel est né en 2015. Cette accélération se retrouve également dans la mise à disposition des accords, puisqu'on peut déjà en consulter une dizaine qui ne démarrent qu'en 2021 - non inclus dans cette étude.

À partir des mouvements décrits en introduction, la dynamique temporelle dessine deux périodes successives de signature des accords (figure 1.1) : tout d'abord, une montée progressive des signatures entre 2015 et $2018^{11}$, passant de 3 à 20 accords ; ensuite une accélération brusque les deux dernières années, avec 91 accords en 2020. Bien que certains soient des "deuxièmes accords" entre un éditeur et un consortium donné pour cette dernière période, la plupart sont nouveaux, avec une diversification des éditeurs et un mouvement d'intensification chez certains consortiums (JISC par exemple) et éditeurs (Springer et Elsevier par exemple).

Figure 1.1 - Répartition des accords par année de début

100

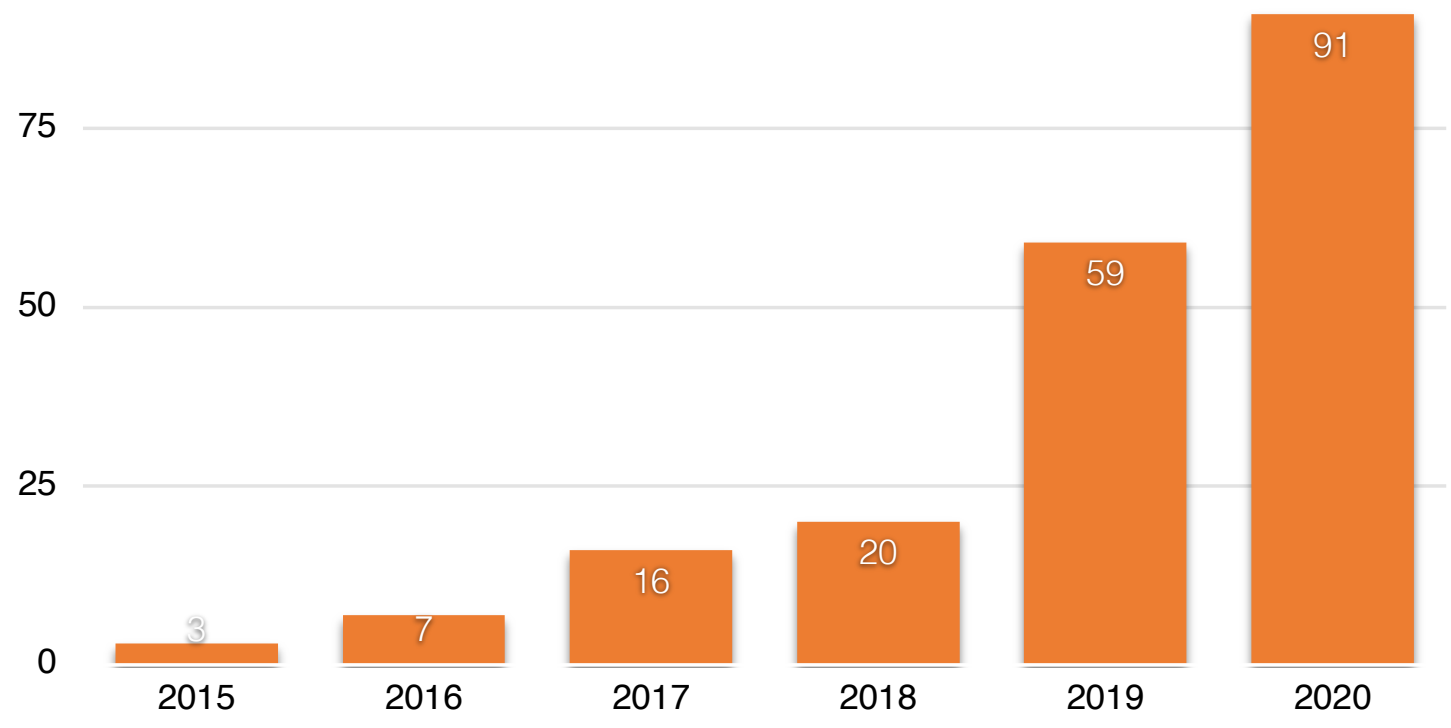

En conséquence, les cinq-sixièmes du corpus de 197 accords constitue le corpus des accords courants (167 au total), raison pour laquelle nous n'avons pas déployé ici de statistiques spécifiques à ce sous-corpus, mais celles-ci sont facilement calculables à partir des données du tableau "TA_List » fourni avec ce rapport. Nous avons au contraire choisi de nous intéresser, dans un premier temps, à toute la période pour quelques éléments quantitatifs.

Deuxièmement, la distribution par éditeurs est marquée par la présence d'un grand nombre de maisons d'édition, puisqu'on en dénombre 39, dont 12 n'ayant signé à notre

\footnotetext{
${ }^{11}$ Si plus de $90 \%$ des accords débutent avec l'année calendaire, cela ne signifie pas pour autant qu'ils ont été signés autour de cette date, en raison de la lourdeur des négociations. Ils sont parfois signés bien en amont (comme ceux de 2021 annoncés dès l'automne) et parfois bien en aval (jusqu'à 6 mois).
} 
connaissance qu'un seul accord ${ }^{12}$. En tête de distribution, comme le montre le tableau 1.1, on retrouve les principaux acteurs du marché de la publication scientifique, que ce soit des maisons commerciales, des presses universitaires ou des sociétés savantes.

Tableau 1.1 - Répartition des accords par éditeurs

\begin{tabular}{|l|c|}
\hline \multicolumn{1}{|c|}{ Éditeur } & Accords (N) \\
\hline Elsevier & 16 \\
\hline Springer Nature & 15 \\
\hline Royal Society of Chemistry (RSC) & 16 \\
\hline Cambridge University Press (CUP) & 14 \\
\hline American Chemical Society & 12 \\
\hline Institute of Physics (IOP) & 12 \\
\hline Taylor \& Francis & 12 \\
\hline
\end{tabular}

Troisièmement, nous avons rassemblé des accords signés dans 15 pays différents, dont 12 européens. Même si la distribution ressemble moins à une courbe de puissance que celle des éditeurs, nous notons bien une forte hétérogénéité entre des pays/consortiums à forte propension de signature d'accords, et d'autres peu signataires. Hors d'Europe, nous avons repéré de nombreux accords aux États-Unis (consortium à l'échelle d'un état fédéré ou encore plus local), au Qatar, en Australie.

Pour l'Europe, la figure 1.2 montre la localisation très inégalitaire des accords avec d'un côté les consortiums d'Europe du Nord et centrale très enclins à signer de tels accords, et de l'autre ceux d'Europe latine en font pour le moment un objet marginal par rapport aux logiques d'abonnement. Il faut noter que si les pays conservant une industrie de la publication scientifique importante (Grande-Bretagne, Allemagne, Pays-Bas) sont très représentés, c'est également le cas pour d'autres qui ne possèdent pas ou plus cette industrie (Scandinavie, Finlande, Hongrie).

\footnotetext{
${ }^{12}$ Nous avons considéré SCOAP3 comme un seul accord, même si plusieurs éditeurs l'ont signé.
} 
Figure 1.2 - Répartition géographique des accords en Europe

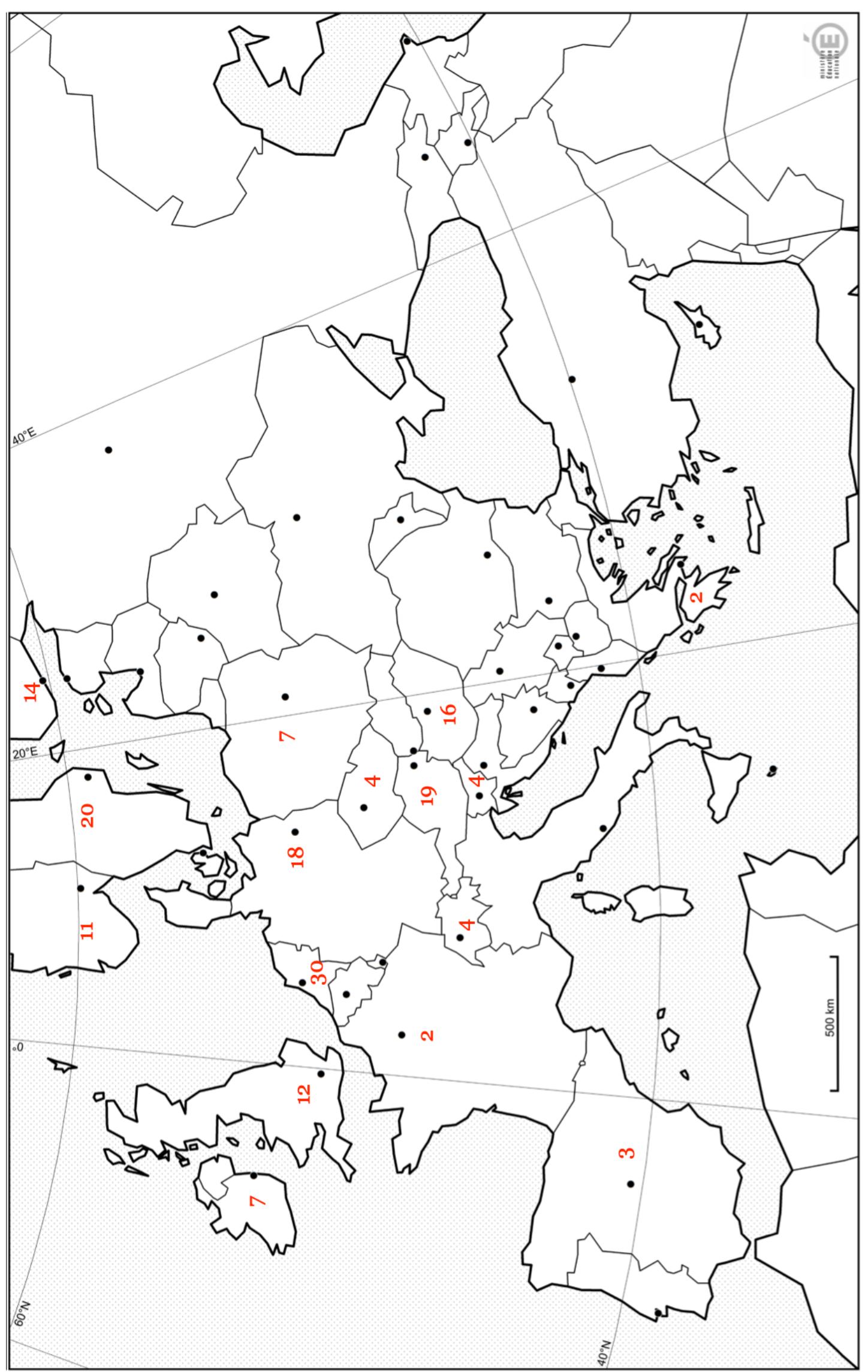


Enfin, la durée nominale des accords, en nombre d'années, est devenu un enjeu important dans la relation entre éditeurs et consortiums, les consortiums cherchant généralement à la raccourcir. Comme l'indique la figure 1.3, deux grands résultats se dégagent de la collection rassemblée. D'une part, on constate une norme émergente remarquable autour des 3 années, qui concerne plus de la moitié des accords. D'autre part, la signature $d^{\prime}$ accords plus longs est marginale, avec $10 \%$ du corpus seulement.

Figure 1.3 - Répartition des accords par durée

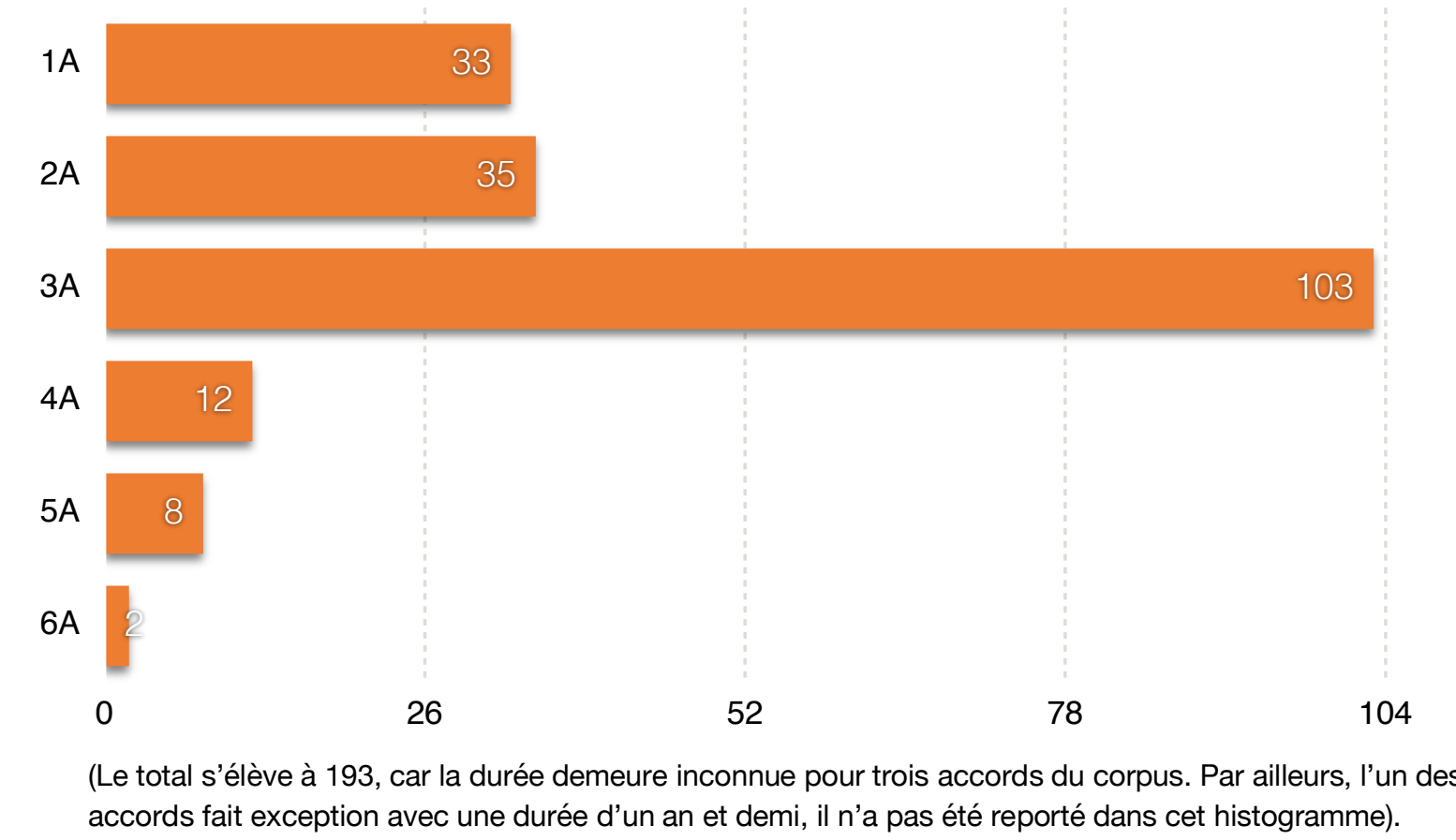

Ajoutons cependant que la variable " durée " n'est pas clairement détachable d'un accord : certains sont marqués par une multitude d'amendements, d'autres par des clauses de sortie (opt-out) pour les établissements signataires (un accord de 3 ans équivaut en fait à $1+1+1)$, voir pour le consortium lui-même, tandis qu'un petit nombre d'accords comprennent des années optionnelles. La figure 1.3 dessine ainsi de grandes tendances, qui pourraient donner lieu à des ajustements à la marge selon les choix de codage.

Une fois posés ces constats généraux, venons-en aux conditions de publicité des documents eux-mêmes.

\subsection{De la culture du secret aux pratiques de transparence}

Comme nous l'avons rappelé en introduction, l'activité de négociation et le résultat de celleci sous la forme d'accords a été longtemps considérée comme privée, voire secrète, que ce soit par le biais de dispositions contractuelles ou par simple pratique professionnelle des négociateurs. Une première ouverture s'est faite, que ce soit du côté des APC, de 
l'abonnement ou des accords étudiés dans ce rapport, sous la pression de demandes d'accès aux documents administratifs ou à l'usage des deniers publics. Suite à cela, les consortiums ont commencé à mettre en place des politiques d'ouverture, elles-mêmes vraisemblablement négociées.

En conséquence, plutôt que de considérer qu'on serait passé d'une période marquée par l'obscurité à une autre régie par la clarté, du secret à la transparence, on peut décrire une gamme de pratiques de transparence, adoptées de manière variable à l'intérieur d'un consortium ou à l'occasion d'un accord donné. Ce faisant, nous n'envisageons pas la transparence comme un principe mais, plus pragmatiquement, comme des formes de matérialité qui influent sur le degré de transparence des accords. Dans le tableau 1.2, nous décrivons cette gamme de pratiques.

Tableau 1.2 - Une gamme de pratiques de transparence

\begin{tabular}{|l|l|}
\hline Référencement de l'accord & Affichage dans ESAC, page dédiée sur le site du consortium \\
\hline Disponibilité du document & Fichier présent sur une URL stable \\
\hline Forme du document & $\begin{array}{l}\text { Format PDF ou DOC, « machine readable », « human } \\
\text { readable » }\end{array}$ \\
\hline Dispositions du document & Intégralité disponible ou parties retirés ou noircies \\
\hline Informations financières & Ensemble des sommes et prix, formules financières \\
\hline Diffusion de l'accord & Référencement par DOI, licence de réutilisation \\
\hline
\end{tabular}

Détaillons quelque peu certains de ces champs et les résultats associés. Pour plus de la moitié des accords sur toute période, et encore une petite moitié en 2020, les documents ne sont pas disponibles. Par définition, si nous les avons repérés, c'est qu'une information minimale était publique - par exemple la durée de l'accord, le mécanisme financier, le périmètre des revues concernées, sans que l'accord ne soit divulgué. De manière peut-être plus surprenante, alors qu'on pourrait s'attendre à ce que des consortiums composés par des personnes formées en Library Sciences soient attentives à cet aspect, la qualité électronique des documents est hautement variable. Hors du JISC qui fournit des documents en " doc ", donc lisibles, explorables et annotables, nous avons découvert que la plupart des fichiers PDF sont simplement des images. De plus, une partie non négligeable d'entre eux est de qualité déplorable, difficilement lisible par des humains, ne parlons pas des machines, puisque nous avons codé $10 \%$ des documents en « mauvaise qualité ». 
En entrant dans le contenu des documents eux-mêmes, on réalise qu'un certain nombre d'entre eux fait l'objet de "coupes" ou de censures, visibles ou invisibles. Ce sont parfois des annexes qui ne sont pas présentes, des pages marquées du sceau «secret industriel " ou du contenu "noirci » ou "blanchi " pour éviter toute lecture tout en conservant leur place dans l'espace du document - nous verrons ce point plus en détail dans la section 2 du rapport. Symétriquement, on cherche parfois des éléments attendus dans l'accord, et on ne les trouve tout simplement pas, sans que cette absence ne soit rendue visible. C'est notamment le cas pour les informations financières, dont le degré de partage est encore plus faible - éléments qui sont repris dans le tableau « Available_TA_TotalSums fourni avec ce rapport. Si la somme totale échangée entre les parties est encore relativement présente, en supposant qu'elle soit fixe ou au moins calculable, le détail des factures pour chaque institution n'est présent que très rarement présent.

Enfin, la dernière ligne du tableau 1.2 ci-dessus évoque la question du statut juridique du document lui-même. S'il est lisible, est-il pour autant stockable, reproductible, réexploitable ? II apparaît paradoxal que l'un des enjeux de ces accords soit de produire des documents en accès ouvert, en particulier au sens de la BOAl, alors que l'accord lui-même demeure fermé, si ce n'est dans son accès, au moins dans sa réutilisation. En effet, nous n'avons repéré que deux accords (ceux du DEAL) qui font l'objet d'un véritablement référencement stable - ici un DOI - et d'une licence associée. Celle- ci a visiblement été obtenue de haute lutte puisque on peut lire dans l'accord DEAL-Springer : "Disclosure of agreement. It is Publisher's position that the terms of this Agreement are proprietary, however the Parties have agreed in this case that the Agreement is placed under a Creative Commons CC-BY-ND 4.0 license and may be made public under this license ". Si la transparence est bien érigée en principe, elle s'oppose encore aujourd'hui en pratique à une culture du secret professionnel et commercial qui transparaît dans les caractéristiques-mêmes de chaque accord.

En conséquence, le degré d'exploration des accords est limité par ces biais d'observation. Dans l'analyse qui suit, nous serons parfois empêchés par l'absence d'information précise sur un point donné dans l'accord publié. Bien entendu, on peut interroger l'existence de biais plus généraux, la révélation de l'accord pouvant entraîner une modification des conditions ou, plus structurellement, être elle-même un enjeu des négociations. Afin d'explorer la possibilité d'analyse de ces biais, nous avons utilisé nos contacts chez Couperin ${ }^{13}$ afin d'avoir accès à des accords "secrets ". Parvenus trop tardivement jusqu'à nous pour être systématiquement comparés au corpus " public » dans

\footnotetext{
${ }^{13}$ Couperin est le consortium qui réunit les bibliothèques universitaires françaises. II a notamment la charge de négocier, pour le compte des bibliothèques, les accords avec les éditeurs scientifiques.
} 
notre rapport, les 15 accords en question feront l'objet d'une lecture différentielle destinée à observer des éléments inédits.

\subsection{Le codage des accords disponibles}

Parmi les 96 accords disponibles au 30 novembre 2020, nous avons pu réaliser une analyse systématique de 62 d'entre eux, qui composent la base empirique centrale de ce rapport. L'impossibilité matérielle d'analyser l'ensemble des accords découle du grand nombre qui ont été rendus disponibles (presque 35) seulement au printemps, voire à l'été, 2020. Plutôt que l'exhaustivité, nous avons choisi de privilégier la diversité en termes de taille, d'éditeurs et de consortiums.

Après une première phase exploratoire, les accords se sont vu systématiquement appliquer le même traitement, dans une logique inductive. En effet, dans un premier temps, nous avons cherché à saturer l'espace de description des accords par la lecture approfondie d'une vingtaine d'entre eux, articulée à la recherche ordonnée de points communs et de différences manifestes. Puis nous avons lu l'ensemble du corpus en produisant systématiquement une fiche-résumé des accords, contenant tous les éléments nécessaires pour la suite du travail. Pour le codage de ces éléments, nous avons ensuite combiné deux voies : une simple extraction des données de l'accord (par exemple le montant d'un « Publish \& Read Fee ", le nombre de revues où l'on peut publier en accès ouvert, la durée de l'accord, etc.), et un codage complexe comme la nature financière de l'accord (voir section 4). Les codages qui sont apparus les plus pertinents après l'analyse font l'objet des deux tableaux de données qui sont remis avec ce rapport (TA_List et Available_TA_TotalSum).

On peut distinguer trois grandes familles de propriétés codées dans les accords, qui sont décrites dans le tableau 1.3 et font l'objet des trois sections suivantes de ce rapport.

Tableau 1.3 - Trois familles de catégories

\begin{tabular}{|l|l|}
\hline Morphologie de l'accord & $\begin{array}{l}\text { Statut, taille, organisation du document, éléments visibles } \\
\text { ou masqués }\end{array}$ \\
\hline Objets de l'accord & $\begin{array}{l}\text { Limites de l'abonnement, définition et périmètre des } \\
\text { publications en OA, infrastructures de production et de } \\
\text { suivi des publications en OA, monde de la publication } \\
\text { inscrit dans l'accord, répartition des coûts entre les } \\
\text { parties. }\end{array}$ \\
\hline Arrangements financiers & $\begin{array}{l}\text { Organisation des APC, formules financières, structure des } \\
\text { montants financiers, évolution programmée des montants }\end{array}$ \\
\hline
\end{tabular}


Si la logique inductive a été dominante, nous avons aussi cherché à tester la pertinence et la cohérence des codages les uns par rapport aux autres, à tenir compte des retours formulés lors de nos différentes présentations au fil de l'étude, et à produire des catégories les plus lisibles à distance. Donnons en deux exemples. Premièrement, parmi les éléments morphologiques les plus frappants, nous avions observé des écarts de taille très importants entre les accords. Ces écarts nous ont conduit à distinguer les (très) longues listes de revues du corps du texte, ou encore le texte principal et les annexes, afin de rendre comparables des accords à la morphologie très différente. Deuxièmement, parmi les éléments financiers les plus immédiats, le coût total de l'accord était une information de base de l'accord Néanmoins, à la recherche systématique de cette information année par année, nous avons réalisé à quel point elle ne pouvait être donnée pour nombre d'accords aux formules financières complexes. L'absence de ce simple chiffrage souligne d'emblée l'incertitude et le risque financier attaché à certaines formes d'accord, que l'on se situe du côté du consortium ou de celui de l'éditeur.

Enfin, précisons que nous avons cherché à développer un vocabulaire constant, qui ne soit pas sémantiquement trop chargé, et qui privilégie la description sur le vocabulaire analytique jargonneux. Pour qualifier les documents, nous avons choisi la notion générique " accord ". Par ailleurs, nous avons généralement désigné la transaction financière entre les deux parties par le terme symétrique de " somme financière " ou de "montant », en évitant ainsi d'endosser le point de vue d'une des deux parties (i.e. le coût pour le consortium, le prix pour l'éditeur). En revanche, l'évaluation monétaire d'une publication en accès ouvert se nomme ici le "prix " ou le "prix unitaire ". Bien que seuls les "auteurs correspondants" soient inclus dans les dispositifs de publication, nous avons choisi par souci de légèreté d'évoquer le plus souvent les " auteurs "; bien qu'un petit nombre de " consortiums " soient en fait des universités ou établissements uniques, nous avons systématiquement désigné la partie payante sous ce terme. 


\section{Section 2.}

\section{Morphologie des accords}

Avant de produire une analyse détaillée des termes de l'accord entre éditeurs et consortium, nous commençons par une caractérisation succincte, mais utile, du genre particulier de documents qui composent notre corpus. Afin d'en prendre la mesure, notre analyse des accords commence par une description de leur morphologie, c'est-à-dire des principaux aspects matériels et de la forme générale qui les caractérisent. Nous commencerons par présenter quelques éléments à propos de la matérialité et de la nature de ces documents (2.1), pour ensuite préciser des caractéristiques de leur taille (2.2). Nous décrirons alors plus directement la structure argumentative des accords, en distinguant plusieurs formes récurrentes dans leur organisation (2.3). En dernier lieu, nous fournirons des exemples empiriques d'accords en donnant à voir la grande diversité de leurs morphologies (2.4).

\subsection{Matérialité et nature des documents}

Les documents que nous avons collectés sont principalement des fichiers PDF (à part les accords JISC, tous au format DOC) qui font entre une petite dizaine de pages et plusieurs centaines (voir plus bas). De qualité inégale, ils suivent un dégradé allant du format texte très lisible à des formats images extrêmement pixellisés. Cette différence de lisibilité n'est pas uniquement liée à la qualité de l'image: certaines parties des documents sont parfois volontairement biffés, certains passages étant masqués par des rectangles noirs (montants, coordonnées des institutions, noms des représentants des parties et leur signature) ou des rectangles blancs surimposés, tandis que des pages sont parfois retirées du document.

Tous ces éléments participent de la politique de la transparence déjà évoquée plus haut, qui est parfois explicitement mise en avant sur le document même, avec des mentions sur le masquage ou le retrait.

Si ces documents renvoient systématiquement à des accords entre un consortium et un éditeur, il est utile d'en distinguer trois types au sein du corpus (tableau 2.1).

1) Premièrement, les accords " de base ", qui constituent l'écrasante majorité du corpus (92\%, 57 documents). Constituant l'accord signé par un consortium et un éditeur pour une période de temps donnée, ces documents peuvent contenir plusieurs éléments. Celui que l'on retrouve systématiquement est évidemment l'accord signé entre un 
consortium et un éditeur, parfois affublé d'une page de garde, et/ou d'un numéro de série. Cependant, certains arborent des passages qui ne relèvent pas de l'accord stricto sensu : c'est le cas des amendements à l'accord, signés durant la période contractuelle (on trouve jusqu'à 6 amendements en plus de l'accord dans le cas néerlandais Elsevier/VSNU 2016), ainsi que des communiqués de presse.

2) Deuxièmement, les accords " étendus " (6\%, 4 documents). II s'agit de documents qui reconduisent un accord de base pour une nouvelle période. Bien qu'ils s'apparentent à un amendement, ces accords étendent l'accord initial au-delà des bornes temporelles initialement prévues. Ces extensions prennent tantôt la forme d'un document propre, tantôt elles sont directement sur le document de l'accord de base.

3) Troisièmement, les "pré-accords" (2\%, 1 document). Le seul document correspondant à ce cas de figure dans le corpus est le Memorandum of Understanding 2020 USA Springer/UC. Le pré-accord est un texte qui présente par anticipation un accord à venir entre une université et un éditeur, en faisant l'hypothèse que la négociation en cours aboutira bel et bien.

Tableau 2.1 - Trois types d'accord

\begin{tabular}{|l|r|r|}
\hline & \multicolumn{1}{|c|}{ N } & \multicolumn{1}{|c|}{$\%$} \\
\hline Accords de base & 57 & 92 \\
- accord seul & 51 & 82 \\
- avec amendements & 5 & 8 \\
\hline Accords étendus & 1 & 2 \\
\hline Pré-Accords & 4 & 6 \\
\hline
\end{tabular}

Compte tenu de leur prépondérance au sein du corpus, lorsque dans la suite de ce rapport nous évoquerons les documents ou les accords, et sauf mention contraire, ce sera pour qualifier les accords « de base ».

\subsection{Taille des documents}

La longueur des documents est variable: le plus long fait 488 pages (France 2019 Elsevier/Couperin) et le plus court 7 pages (Netherlands 2019 IWA/Delft-Wageningen). Entre ces deux extrêmes, une dizaine font plus de 100 pages (principalement des accords Springer) et une dizaine moins de 30 pages. La longueur moyenne est de 70 pages, tandis que la 
médiane se situe à 48 pages (cf. tableau 2.2). Autrement dit, le " même " objet contractuel prend des formes textuelles contrastées, résultant des multiples éléments assemblés et des degrés de précision extrêmement variables d'un document à l'autre.

Une manière de rendre compte des différences de taille entre ces documents consiste à se référer à la nature et à l'organisation des clauses contractuelles: tous les accords n'arborent pas exactement le même contenu juridique ni le même plan (voir plus bas) ; sans parler de l'espacement des lignes, des marges, de la police et du corps des caractères. Pour autant, à la lecture, certains éléments, qui ne sont pas à proprement parler des clauses des accords, jouent directement sur sa taille.

\subsubsection{Les listes de revues}

Le principal élément « hors clauses contractuelles » (bien que partie intégrante de l'accord) qui influe sur la longueur des documents consiste dans des listes de revues. Tous les accords signés renvoient d'une manière ou d'une autre à des revues, que ce soit dans le cadre de l'abonnement ou celui de la publication en accès ouvert (hybrides et full $O A^{14}$ ). Dans de nombreux cas (72\% des accords), ces revues sont mentionnées sous la forme de listes, annexées à l'accord. Comme le montre le tableau 2.2, leur taille est elle-même très variable, comprise entre une simple page (e.g. UK 2020 The Compagny of Biologists/JISC) et 171 pages (e.g. Sweden 2018 TandF/Bibsam).

Tableau 2.2 - Taille des documents et part des listes de revues exprimées en nombre de pages

\begin{tabular}{|l|r|r|r|r|}
\hline & $\begin{array}{c}\text { Taille totale } \\
(\mathrm{N})\end{array}$ & $\begin{array}{l}\text { Taille hors listes } \\
\text { de revues }(\mathrm{N})\end{array}$ & $\begin{array}{c}\text { Listes de } \\
\text { revues (N) }\end{array}$ & $\begin{array}{c}\text { Ratio taille liste / } \\
\text { taille totale }\end{array}$ \\
\hline Valeur la plus haute & 488 & 383 & 171 & $2 \%$ \\
\hline Valeur la plus basse & 7 & 6 & 0 & $82 \%$ \\
\hline Moyenne & 70 & 45 & 25 & $24 \%$ \\
\hline Médiane & 48 & 31 & 5 & $15 \%$ \\
\hline
\end{tabular}

Cette variation découle non seulement des bouquets de revues inclus dans la négociation entre les parties, mais également du périmètre des éditeurs, certains ayant peu de revues ( 3 pour The Compagny of Biologists) tandis que d'autres en ont plus de 2000 (comme Elsevier ou Springer). En conséquence, les documents qui n'affichent pas de liste de

\footnotetext{
14 Pour rappel, les revues hybrides proposent des contenus sous abonnement ainsi que des contenus en accès ouverts. En revanche, les revues dites « full $O A$ » publient exclusivement des articles en accès ouvert.
} 
revues ne dépassent jamais 60 pages. Et parmi les accords qui disposent de listes de revues, celles-ci constituent en moyenne $30 \%$ du document total.

\subsubsection{Les listes d'institutions, amendements et communiqués de presse}

D'autres éléments « hors clauses contractuelles » jouent sur la longueur des documents, mais ils sont nettement moins imposants et plus ponctuels. C'est le cas du retrait de certaines pages, qui est majeur pour les variations à la baisse. Par exemple, les accords Norway 2020 Springer/Unit et Sweden 2020 Elsevier/Bibsam font respectivement 20 et 22 pages, alors que leur pagination indique 137 et 143 pages. Pour les variations à la hausse, deux éléments peuvent être soulignés : d'une part, les listes d'institutions bénéficiant de l'accord, presque toujours présentes en annexes, font généralement quelques pages tout au plus. Dans certains cas, elles sont néanmoins beaucoup plus longues, à l'image de l'accord France 2019 Elsevier/Couperin qui constitue un cas extrême à cet égard, avec des listes d'institutions sur près de 45 pages. D'autre part, la présence d'amendements à l'accord (avec un autre cas extrême : l'accord Sweden 2018 T\&F/Bibsam dont un amendement de 72 pages, incluant également une liste de revues), et les communiqués de presse, qui ne font pas partie de l'accord stricto sensu, ont tendance à augmenter la taille du document.

\subsection{Structure des documents}

Autre aspect important dans la variation des documents : la structure organisationnelle des termes de l'accord. Au sein du corpus, deux principales formes d'organisation des documents sont repérables. La première opère une bipartition, quand la seconde renvoie à un format commun systématiquement appliqués par certains éditeurs.

\subsubsection{Corps de l'accord et annexes}

Pour une large majorité d'accords (78\%), on observe une organisation en deux parties. Une première, qui fait en général une dizaine de pages, et qui correspond à ce que l'on pourrait appeler le « corps » de l'accord. Une seconde, nettement plus longue, qui est constituée des annexes de l'accord. Cette structure en deux parties ne dit toutefois rien de l'intérêt des éléments qu'elles abritent. Le corps et les annexes sont d'égale importance pour la compréhension de l'accord. Par ailleurs, dès que l'on rentre dans le détail de chacune des parties, aucun plan type ne se dégage. Chaque accord se présente plutôt comme un agencement de thèmes et d'objets plus ou moins récurrents, dont la plupart ne sont pas organisés de la même manière d'un document à l'autre. Examinons cependant ce qui se trouve habituellement dans chacune des deux parties. 
Le corps de l'accord prend place immédiatement sous le titre du document. II démarre par l'évocation du nom et du statut des parties, et se poursuit souvent par un ensemble de définitions des termes de l'accord. Viennent ensuite des considérations sur la durée de l'accord, les obligations des parties concernant la mise à disposition et l'usage des contenus sous abonnement (performances de l'éditeur, restrictions d'usage des institutions, etc.), des éléments éventuels sur l'OA, l'identité du payeur (il s'agit souvent du consortium, plus rarement des institutions directement), les échéances de paiement, et enfin des clauses génériques du droit des contrats (résiliation en cas de non-respect des clauses, force majeure, garanties, etc.). Cette première partie s'achève systématiquement par les dates et signatures manuscrites des parties.

Les annexes (indifféremment appelées "Annex", "Schedules", ou encore "Appendix »), dont le nombre varie (5 en moyenne), constituent la partie la plus longue du document. On y trouve la plupart du temps les informations suivantes (dans un ordre variable): des listes d'institutions membres du consortium signataire (avec adresses physiques et adresses IP) ; des explications sur les objets de l'accord, les montants, et le mécanisme financier (avec éventuellement une répartition des frais par institution); des dispositions précises liées à l'accès ouvert (dont les règles de gestion du " workflow ») ; des listes de revues (accessibles sous abonnements, hybrides et full OA dans lesquelles il est possible de publier). De manière plus ponctuelle, les annexes proposent parfois des passages sur le type de licence en accès ouvert, la politique de protection des données des institutions, la fouille de textes et de données ("text and data mining "), ou encore la "sign-up letter ${ }^{15}$ que doivent signer les institutions.

Une variante de cette organisation est repérable (pour 16 accords) : tandis que le corps de l'accord circonscrit les relations contractuelles entre l'éditeur et le consortium, la relation entre l'éditeur et les institutions fait l'objet d'une annexe en propre (sous le titre " model licence agreement ») dans la deuxième partie. Bien que partiellement redondante avec la première partie (e.g. rappel du nom des parties, définitions, clauses contractuelles génériques), cette annexe précise les droits et devoirs spécifiques des institutions et de leurs membres.

\subsubsection{La marque des éditeurs et des consortiums}

Certaines régularités dans la structure des accords sont liées aux éditeurs, qui appliquent un format commun d'un accord à l'autre. Dans notre corpus, c'est plus particulièrement visible chez Springer. La « marque » Springer se différencie de la structure en deux parties à plusieurs

\footnotetext{
15 Les accords transformants sont généralement négociés entre un éditeur et un consortium national. Cependant, pour que l'accord soit effectif auprès des membres du consortium, chacun d'eux doit à son tour signer un accord bilatéral qui reprend les termes de la négociation. Cet accord bilatéral se présente souvent sous la forme d'une «sign-up letter », que l'on peut traduire par lettre d'engagement.
} 
égards. Tout d'abord, le document commence par un résumé de quelques pages à propos des objets de l'accord (abonnement et accès ouvert), résumé qu'on ne retrouve quasiment jamais ailleurs (sauf dans Netherlands 2017 CUP/VSNU). Ensuite, juste sous le résumé, on trouve les " product terms " qui s'organisent systématiquement autour d'une " Part $A$ " consacrée à la publication en accès ouvert, une "Part $B$ » dédiée à l'abonnement, souvent assortie d'une longue liste de revues, et parfois une " Part $C$ » précisant les modalités d'entrée et de sortie de l'accord ("opt-in » et «opt-out »). Enfin, après les annexes, le document se termine par les " general conditions for electronic products", soit des considérations génériques de droit des contrats, que l'on trouve plutôt au début dans les autres accords.

Outre le cas spécifique de Springer, on repère, mais dans une moindre mesure, des régularités d'éditeurs au sein de la structure en deux parties. Par exemple, chez Wiley, les annexes se suivent généralement dans le même ordre : informations sur les institutions, liste de revues, publishing, informations financières. De manière similaire pour un éditeur de taille plus modeste comme Brill, après la première partie, les annexes enchaînent la liste des institutions, la description des objets de l'accord, puis le "model licence agreement". Cependant, cette régularité n'est pas systématique : chez certains éditeurs comme Elsevier ou Sage, les accords signés sont organisés selon une structure variable, sans pour autant être radicalement différente. Ce sont plutôt des aménagements spécifiques d'une trame partagée. Difficile cependant de savoir si cette variation est une décision unilatérale de l'éditeur, ou une conséquence de la négociation et de l'ajustement des conditions de l'accord qui ont mené à la signature d'un accord particulier.

Par ailleurs, on peut signaler deux cas où les consortium (et non plus les éditeurs) imposent une structure au document, même si ces situations sont beaucoup plus rares. D'une part, le consortium hongrois EISZ, puisque chaque accord qu'il a signé avec différents éditeurs propose une première partie bilingue (magyar/anglais) qui suit rigoureusement le même plan, avant de laisser la place au format éditeur dans les annexes. D’autre part, le consortium français Couperin, dont l'accord signé avec Elsevier en 2019 (France 2019 Elsevier/Couperin) suit un plan atypique, qui déroge à la fois au format classique d'Elsevier et à la structure en deux parties, avec 19 articles et 15 annexes.

Le tableau 2.3 synthétise les différentes formes d'organisation des documents que nous avons décrits dans cette section. 
Tableau 2.3 - Les différentes structures des documents

\begin{tabular}{|l|c|c|c|c|}
\hline & $\begin{array}{c}\text { Structure en } \\
\text { deux parties }\end{array}$ & Structure Springer & $\begin{array}{c}\text { Deux parties avec } \\
\text { marque du } \\
\text { consortium EISZ }\end{array}$ & Structure atypique \\
\hline Accords (N) & 47 & 9 & 6 & 1 \\
\hline Accords (\%) & 78 & 15 & 10 & 2 \\
\hline Exemples & $\begin{array}{l}\text { Norway 2020 } \\
\text { Sage/Unit }\end{array}$ & $\begin{array}{l}\text { Germany 2020 } \\
\text { Springer/Projekt Deal }\end{array}$ & $\begin{array}{l}\text { Hungary 2019 } \\
\text { RSC/EISZ }\end{array}$ & $\begin{array}{l}\text { France 2019 } \\
\text { Elsevier/Couperin }\end{array}$ \\
\hline
\end{tabular}

\subsection{Cas empiriques}

Afin de rendre la morphologie des accords plus tangible, dans cette section, nous avons sélectionné plusieurs cas empiriques qui déclinent les principales variations identifiées dans la composition matérielle des documents. Nous illustrerons d'abord les deux principales structures des documents (celle en deux parties et celle typique de Springer), avant de déployer la diversité des morphologies des accords, sous forme d'un tableau plus général.

\subsubsection{Cas $n^{\circ} 1$ : la structure en deux parties}

L'accord Netherlands 2019 IOS Press/VSNU est un document PDF de 31 pages au format texte, de qualité moyenne, sans page de garde, avec pagination. Figurent en en-tête à gauche le logo de SURFmarket (qui signe l'accord au nom du consortium néerlandais VSNU) et à droite celui de l'éditeur IOS Press. En pied de page, on trouve à droite la mention "agreement for intermediary services content. IOS Press 2019-2020", et à gauche, des champs paraphés ( initials SURFmarket» et "initials Publisher »). Les signatures sont masquées par un rectangle noir, ainsi que les montants individuels par institution.

Le document arbore la structure caractéristique en deux parties : le corps de l'accord (pages 1 à 9) et les annexes (5 au total, ici appelées "Schedules », entre les pages 10 et 31). Voici la structure détaillée de son organisation :

-[Agreement], p. 1-9.

-Schedule A - Categories of institutions, p. 10.

-Schedule B-Description of licensed materials, with prices and licence models, $p .11-12$.

-Schedule C-Model licence agreement, p. 13-19.

-Schedule C-a: Licensed materials, types of licence and licence fees, p. 20-21.

-Schedule C-b: Terms and conditions for open access publishing for authors, $p .22$.

-Schedule C-c: Support, p. 23.

-Schedule C-d-e: Detailed list of licensed materials with access rights and with 
publishing rights, p. 24-27.

-Schedule D-Access and availability licensed materials, p. 28-29.

-Schedule E-Text and Datamining, p. 30-31.

Le corps de l'accord décline différents éléments sur le nom des parties et leurs obligations, les définitions de l'accord, les modalités de paiement, et les clauses générales de droit des contrats (" guarantee ", " premature termination or dissolution ", " indivisibility", etc.).

En annexes, les objets régulièrement présents dans ce genre de document sont agencés de la manière suivante : la liste d'institutions membres (Schedule A), la description des objets de l'accord (Schedule B), les obligations de performance de l'éditeur quant à l'accès au contenu (Schedule D), et des considérations sur la fouille de textes et de données (Schedule E).

Cet accord constitue toutefois une variante de la structure classique en deux parties : les relations entre l'éditeur et les institutions ne sont pas dans le corps de l'accord, mais dans une annexe (Schedule $C$ ). Celle-ci répète des éléments déjà présents dans le corps de l'accord, mais également dans d'autres annexes (le Schedule C-a sur les objets de l'accord est un copier/coller du Schedule B). Elle introduit également des éléments nouveaux comme la question de la publication en accès ouvert (Schedule C-b), les supports fournis par l'éditeur (Schedule C-c), et la liste des revues sous abonnement et en OA (Schedule C-d-e).

\subsubsection{Cas $n^{\circ} 2$ : la structure "Springer »}

L'accord Finland 2018 Springer/Finelib se présente sous la forme d'un document PDF de 156 pages au format texte, de bonne qualité, avec un identifiant en pied de page (25591) et une pagination. Les pages 18 à 144 sont dédiées à des listes de revues. Des éléments sont masqués, dont la ventilation des montants par institution ou les adresses IP des membres. Le masquage passe ici non pas par des rectangles noirs mais par un effacement, systématiquement mentionné par un texte rouge encadré. Voici comment se décline la structure typique d'un accord Springer :

-License agreement, p. 1-8.

-Product terms: Compact, p. 9

-PART A: Open access publishing, p. 9-14.

-PART B: Access to content, [avec du texte p. 15-18, et des listes p. 18-75], p. 15-75.

-Schedule 1: Open choice journals [liste de revues], p. 76-144.

-Schedule 2: APC rates, p. 145.

-General terms and conditions for electronic products, $p$ 146-156. 
Les premières pages, consacrées au "licence agreement ", résument le contenu de l'accord. Outre des éléments de caractérisation des parties, et des précisions sur la durée et les modalités de paiement, les premières pages affichent un tableau récapitulatif des objets de l'accord et des montants en jeu. Ensuite, les "product terms » entrent dans le détail de deux objets bien délimités, à savoir la publication en accès ouvert (PART A) et l'accès à des revues sous abonnement (PART B).

Deux annexes (Schedules) constituent la suite de ce document : l'une correspond à la longue liste de revues (Schedule 1) concernées par cet accord, et l'autre précise les frais et modalités d'usage des APC (Schedule 2). Enfin, l'accord se termine par les « general terms and conditions ". Cette rubrique abrite des clauses que l'on trouve classiquement au début des accords marqués par la structure en deux parties : définitions, droits et obligations des parties, éléments de droit des contrats sur la résiliation, les garanties, etc.

\subsubsection{Diversité des morphologies}

Le tableau 2.4 ci-dessous répertorie un certain nombre d'accords issus de notre corpus. Classée par taille de document, cette sélection donne à voir la diversité des morphologies des documents que nous avons décrites jusqu'ici. Elle décline ainsi les considérations sur la composition matérielle des documents (qualité de l'image et éléments masqués), sur le type d'accord (de base, étendu, pré-accord), sur la taille (du document, de la liste de revues, amendements à l'accord stricto sensu), et enfin sur les différentes structures des documents 
Tableau 2.4 - Une diversité de morphologies

\begin{tabular}{|c|c|c|c|c|c|c|c|}
\hline Nom de l'accord & 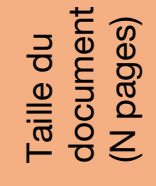 & 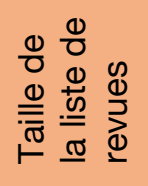 & Type d'accord & Lisibilité & Éléments masqués & $\begin{array}{l}\text { Éléments } \\
\text { ajoutés }\end{array}$ & Structure de l'accord \\
\hline USA 1010 Springer/UC & 8 & 0 & Pré-accord & Bonne & Non & / & 1 \\
\hline $\begin{array}{l}\text { Netherlands } 2020 \\
\text { CUP/VSNU-UKB }\end{array}$ & 14 & 9 & Accord étendu & Moyenne & Non & / & 1 \\
\hline $\begin{array}{l}\text { Sweden } 2020 \\
\text { Elsevier/Bibsam }\end{array}$ & 22 & 0 & Accord de base & Bonne & $\begin{array}{l}\text { Pages retirées (143 } \\
\text { pages à l'origine) }\end{array}$ & Non & Structure en deux parties \\
\hline $\begin{array}{l}\text { Netherlands } 2017 \\
\text { CUP/VSNU }\end{array}$ & 47 & 0 & Accord de base & Mauvaise & Non & $\begin{array}{c}1 \\
\text { amendement }\end{array}$ & Structure en deux parties \\
\hline $\begin{array}{l}\text { Netherlands } 2016 \\
\text { Elsevier/VSNU }\end{array}$ & 51 & 0 & Accord de base & Moyenne & Non & $\begin{array}{c}6 \\
\text { amendement } \\
s\end{array}$ & Structure en deux parties \\
\hline UK 2020 ERJ/JISC & 64 & 1 & Accord de base & Bonne & $\begin{array}{l}\text { Prix retirés avec } \\
\text { mention }\end{array}$ & Non & $\begin{array}{l}\text { Structure en deux parties avec } \\
\text { le contrat institutionnel en } \\
\text { annexe }\end{array}$ \\
\hline $\begin{array}{l}\text { Hungary } 2019 \\
\text { Elsevier/EISZ }\end{array}$ & 68 & 46 & Accord de base & Moyenne & $\begin{array}{l}\text { Coordonnées des } \\
\text { institutions retirées }\end{array}$ & Non & $\begin{array}{l}\text { Structure en deux parties avec } \\
\text { format consortium }\end{array}$ \\
\hline $\begin{array}{l}\text { Austria } 2019 \\
\text { Springer/KEMO }\end{array}$ & 128 & 105 & Accord de base & Bonne & Non & Non & Structure « Springer » \\
\hline France 2019 Elsevier & 488 & 105 & Accord de base & Bonne & Non & Non & Structure atypique \\
\hline
\end{tabular}




\section{Section 3.}

\section{S'abonner aux revues et publier en accès ouvert}

Après une exploration morphologique des accords, nous proposons de les examiner plus en détail sous un autre angle. Dans cette section, nous rendrons plus spécifiquement compte des deux principaux objets qui sont au centre des négociations entre les parties prenantes : d'une part, les modalités d'abonnement à des supports scientifiques (3.1), d'autre part, les conditions de la publication en accès ouvert dans des revues (3.2).

\subsection{Les conditionnements de l'abonnement}

L'abonnement à des publications scientifiques est une forme traditionnelle qui organise les relations entre éditeurs et institutions de recherche depuis des décennies. En dépit l'importante digitalisation des productions scientifiques au cours des trente dernières années, sa place est demeurée centrale. Qu'en est-il cependant avec la promotion croissante de l'accès ouvert ? Et surtout, à quelle(s) forme(s) d'accord l'abonnement est-il soumis au cœur des transactions contemporaines?

Afin d'esquisser des pistes de réponse, nous commencerons par étudier la définition de l'abonnement telle qu'elle est inscrite dans les accords. Nous verrons que cette définition tourne autour de l'idée d'accès à des titres (3.1.1). Nous examinerons ensuite les droits et devoirs attachés à l'abonnement (3.1.2), que nous proposerons finalement de résumer autour de deux préoccupations majeures : les modalités de l'accès et de la sécurité (3.1.3).

\subsubsection{Accéder à des revues}

Les éléments constitutifs de l'objet « abonnement " sont relativement standardisés dans les accords. À ce titre, ils se déclinent sous des formes lexicales redondantes, telles que " subscribed products ", "licenced materials", " mise à disposition de bases de données", ou encore "subscription and access to electronic databases". Qu'il s'agisse de bases de données, de produits ou matériaux informatifs, tous font de l'accès le cœur des modalités de l'abonnement.

Cette importance de l'accès pointe plus précisément vers deux éléments. Premier élément, l'abonnement concerne systématiquement des listes de revues, qui sont 
habituellement présentées en annexe de l'accord ${ }^{16}$. Comme nous l'avons exposé dans la section " morphologie » de ce rapport, les listes de revues sont de tailles variables, à hauteur de l'offre de chaque éditeur. Ainsi, un éditeur comme European Respiratory Journal n'a qu'une seule revue à proposer dans son accord avec JISC (UK 2020 ERJ/JISC), tandis qu'un gros éditeur comme Springer en arbore généralement plus de 1000 - le plafond de notre corpus étant 2200 revues dans le cadre de l'accord à venir 2020 Springer/UC. De manière générale, la composition de la liste de revues négociées est elle aussi variable d'un accord à l'autre. Elle correspond soit à l'ensemble des revues d'un éditeur (surtout chez les éditeurs de petite et moyenne taille, à l'image d'IOP qui loue l'accès à ses 44 revues), soit à une ou plusieurs collections qui existent indépendamment de l'accord (plutôt chez les gros éditeurs, comme la "Complete Freedom Collection" d'Elsevier ou la collection "Sage Premier »). Les revues concernées par l'abonnement sont généralement rassemblées dans une annexe de l'accord, presque systématiquement présentée sous la forme d'un tableau qui recense pour chaque revue un certain nombre d'informations : notamment, le titre de la revue, un identifiant, un ISSN papier et un ISSN électronique, plus épisodiquement la date de création de la revue, un code éditeur et un code de consortium.

Par exemple, l'accord néerlandais 2020 Walter De Gruyter/VSNU-UK inclut, entre les pages 23 et 32, une annexe intitulée "Schedule $C-b$ : Detailed list of licensed material with access rights ". Nous avons reproduit ici les premières lignes de ce tableau, qui liste les revues souscrites, avec un titre, un ISSN online, un code éditeur ("WdG code ") et un code consortium ("SURF code »).

\begin{tabular}{|l|l|l|l|}
\hline Journal Name & ISSN Online & WdG Code & $\begin{array}{l}\text { SURF } \\
\text { Code }\end{array}$ \\
\hline ABI Technik & $2191-4664$ & ABITECH & 26.718 \\
\hline Accounting, Economics, and Law: A Convivium & $2152-2820$ & AEL & 26.719 \\
\hline Advanced Nonlinear Studies & $2169-0375$ & ANS & 31.899 \\
\hline Advanced Optical Technologies & $2192-8584$ & AOT & 26.739 \\
\hline Advances in Calculus of Variations & $1864-8266$ & ACV & 27.323 \\
\hline
\end{tabular}

Source : Netherland 2020 Walter De Gruyter/NSNU-UKB, Schedule C-b, p.23.

Autre exemple, tiré d'un de l'accord Finland 2018 Springer/Finelib. Ici, la liste de revues n'est pas en annexe, mais à la fin de la «Part B " portant sur l'abonnement (" access to content ») sous la clause « 4. Content ». Relativement longue (elle s'étend entre les pages 18 et 75), elle se présente également sous la forme d'un tableau qui recense la place de la revue dans la liste (une manière de compter le nombre total de revues accessibles -2215 en

\footnotetext{
${ }^{16}$ Dans notre corpus, seul le cas France 2019 Elsevier/Couperin constitue une exception. Certes cet accord propose aux institutions membres du consortium un abonnement à différentes collections listées en annexes, mais également la possibilité d'un abonnement « titre-à-tire ", c'est-à-dire à n'importe quelle revue distribuée par Elsevier.
} 
l'occurrence), le titre de la revue, son numéro d'identification, son ISSN, l'année de démarrage (celle à partir de laquelle l'accord donne accès) et le volume de démarrage (celui auquel la revue donne accès).

\begin{tabular}{|r|r|l|l|c|c|}
\hline \multicolumn{1}{|c|}{ Title } & $\begin{array}{c}\text { ISSN } \\
\text { electronic }\end{array}$ & Start Year & Start volume \\
\hline 1. & 13205 & 3 Bitlech & $2190-5738$ & 2011 & 1 \\
\hline 2. & 13319 & 3D Research & $2092-6731$ & 2010 & 1 \\
\hline 3. & 10288 & 4 OR & $1614-2411$ & 2003 & 1 \\
\hline 4. & 12248 & The AAPS Journal & $1550-7416$ & 1999 & 1 \\
\hline 5. & 12249 & AAPS PharmSciTech & $1530-9932$ & 2000 & 1 \\
\hline 6. & 261 & Abdominal Radiology & $2366-0058$ & 1997 & 22 \\
\hline 7. & 12188 & $\begin{array}{l}\text { Abhandlungen aus dem Mathematischen Seminar der } \\
\text { Universität Hamburg }\end{array}$ & $1865-8784$ & 1997 & 67 \\
\hline
\end{tabular}

Source : Finland 2018 Springer/Finelib, Part B access to content, 4. Content, p. 18

Deuxième élément, l'abonnement est associé à un périmètre spécifique d'accès aux revues listées en annexe. Une formule de base consiste à garantir l'accès à ces revues durant toute la période de l'accord. Pour autant, cette formule de base peut être modulée de trois manières distinctes. Tout d'abord, l'abonnement aux revues peut répartir l'accès entre plusieurs bouquets indépendants. Comme le montre le tableau 3.1, c'est le cas pour 10 accords, soit 16\% du total. Par exemple, l'accord 2018 Sweden TandF/Bibsam distribue les revues en cinq bouquets, qui délimitent des collections disciplinaires et temporelles : SSH and S\&T 2018 Collection; SSH and S\&T 2015 Collection ; Medical Library 2018 Collection ; Medical Library 2015 Collection; FRESH Collection 2018. Chaque institution du consortium peut alors souscrire un abonnement à l'une ou l'autre de ces collections, ainsi qu'à l'ensemble.

Tableau 3.1 - Distribution différentielle de l'accès aux revues

\begin{tabular}{|l|c|c|}
\hline \multicolumn{1}{|c|}{ Liste de revues } & Accords (N) & \% \\
\hline Accès par bouquets & 10 & 16 \\
\hline Accès complet & 52 & 84 \\
\hline
\end{tabular}

Ensuite, l'abonnement peut inclure un accès aux archives des revues, c'est-à-dire la mise à disposition des articles publiés dans les revues listées avant la date de démarrage de l'accord. L'archive est tantôt évoquée dans une clause à part entière de l'accord, tantôt au sein de l'annexe portant sur les revues souscrites. Elle est explicitement mentionnée dans 34 accords (57\% du corpus).

Enfin, l'abonnement aux revues peut être converti en accès perpétuel, une fois la période de l'accord achevée. On retrouve généralement cette disposition dans le corps de 
l'accord, sous la forme d'une clause intitulée "continuous access" ou "post-termination access ». On recense 43 accords mentionnant l’accès perpétuel (69\% du corpus).

\subsubsection{Droits et obligations des parties : usagers, institutions, éditeur}

L'abonnement aux revues est régi par un ensemble de droits et d'obligations pour les différentes parties : généralement, les utilisateurs autorisés, les institutions, l'éditeur ; mais rarement le consortium, qui fait figure d'intermédiaire et ne fait pas directement usage des revues. Des traces de cette réglementation sont repérables dans les clauses au sein du corps de l'accord, dans l'annexe " model licence agreement " lorsqu'il y en a une, et dans l'annexe finale pour les accords Springer ("general conditions for electronic products »). Le mode de présentation des droits et devoirs des parties consiste souvent à lister ce qu'elles peuvent, ne peuvent pas, et éventuellement doivent faire - sachant que l'ordonnancement documentaire de l'éditeur, de l'institution et de l'usager varie d'un accord à l'autre.

Prenons l'exemple de l'accord suédois de 2020 entre Elsevier et Bibsam. Dans le corps $\mathrm{du}$ document, les trois premières clauses (Section 1: Subscription; Section 2 : Elsevier performance obligations; Section 3 : Subscriber perfromance obligations) nous apprennent les choses suivantes :

1) les utilisateurs autorisés peuvent accéder aux revues, télécharger les articles, les imprimer, les conserver etc. ; mais ils ne peuvent pas les modifier, créer des dérivés, enlever ou modifier les copyrights, télécharger de manière systématique les articles (à l'aide de robots par exemple).

2) Les institutions qui souscrivent peuvent utiliser les métadonnées, diffuser les résumés sur les interfaces utilisateurs etc. ; et elles doivent assurer l'identification des usagers autorisés, lutter contre les accès non autorisés en installant notamment des accès sécurisés par mot de passe, informer rapidement Elsevier d'usages non autorisés (sous peine de suspension du service).

3) L'éditeur assure l'accès aux produits souscrits, l'accès doit être assuré $99 \%$ du temps (avec $1 \%$ du temps dédié à la maintenance), se conforme à différents droits et licences (e.g. Web Content Access Guidelines, Standardized Usage Statistics Harvesting Initiative, Open URL Standard, TRANSFER code of practice, KBART standard, etc.), fournit chaque année la liste des titres disponibles aux institutions tous les 25 septembre et conserve la propriété sur les produits. Cette liste n'est pas exhaustive, et elle peut varier d'un accord à l'autre, avec parfois des considérations sur la possibilité d'intégrer ou non l'accord en cours de route («opt-in »), ou l'autorisation de faire de la fouille de textes et de données.

On peut supposer (sans pouvoir l'affirmer, faute d'accès aux sources) qu'un tel ordonnancement est largement hérité des accords d'abonnement traditionnels que les 
éditeurs et les universités (voire les consortiums) signaient avant l'émergence des accords transformants, dans la deuxième moitié des années 2010.

\subsubsection{Deux préoccupations majeures : accès et sécurité}

Une manière possible de résumer les droits et devoirs des parties consiste à souligner deux préoccupations majeures à propos de l'abonnement aux revues. La première concerne l'accès aux titres et leurs articles. Ainsi, les institutions doivent fournir des adresses IP afin $d^{\prime}$ authentifier les utilisateurs autorisés. Ces adresses IP se trouvent généralement dans une annexe à part qui recense les institutions, et sont souvent masquées. Par ailleurs, l'éditeur est juridiquement tenu d'assurer l'accès pérenne aux revues, en garantissant notamment la capacité d'accueil de ses serveurs, et en minimisant les moments de maintenance.

La seconde a trait au caractère sécurisé de l'accès. Cet aspect se décline ici de plusieurs manières :

1) sécurité garantie par l'éditeur des données utilisateurs, notamment les adresses IP fournies;

2) sécurité garantie par l'éditeur lors de la consultation des titres sur internet (via des serveurs sécurisés par mot de passe);

3) sécurité garantie par les institutions vis-à-vis de la diffusion d'éléments à des tiers. Ce sont toutes les restrictions d'usage comme l'interdiction du téléchargement massif, l'interdiction des utilisations commerciales, et l'obligation de prévenir l'éditeur en cas d'accès non autorisés ;

4) sécurité garantie par le consortium et les institutions de ne pas revendiquer la propriété des titres appartenant à l'éditeur. Les accords insistent systématiquement sur le fait que les contenus sont bel et bien la propriété de l'éditeur, et que l'accord ne constitue en aucun cas une cession des contenus, avec la mention «non-transferable and non-exclusive rights $"$.

Au vu de ces différentes caractéristiques, la partie abonnement des accords relève donc d'un monde fermé, dont l'accès est soumis à un accord payant qui déclenche des séries d'identification des circulations légitimes tant du contenu informationnel que des usagers. Les interdictions de réutilisation, ou même de copie, qui sont au cœur de ces dispositions de l'accord sont à l'opposé des définitions communes de l'accès ouvert, qui constitue pourtant le second objet des mêmes accords.

\subsection{La publication en accès ouvert}

Le second objet des négociations au centre des accords concerne la publication en accès ouvert. Nous verrons d'abord que sa mise en place concrète au sein des accords implique de 
nombreuses considérations autour de la gestion du workflow de publications (3.2.2). Ensuite, nous montrerons que les caractéristiques des publications qui circulent au sein de ce workflow peuvent prendre des formes extrêmement variées (3.2.2.).

\subsubsection{La gestion du workflow}

À l'instar de l'abonnement, la publication en accès ouvert s'accompagne d'un certain nombre de préoccupations quant à ses conditions pratiques de mise en œuvre. Ces préoccupations renvoient à un problème inédit pour les bibliothèques et les éditeurs traditionnels : il ne s'agit plus seulement de réglementer l'accès aux revues et d'assurer la sécurité matérielle et juridique des parties (comme dans la formule classique de l'abonnement), mais de gérer un flux de publications en accès ouvert. En pratique, il s'agit donc de définir le circuit par lequel un article publié par un auteur peut effectivement être ouvert, ou peut être ouvert sans facturation à l'auteur, dans le cadre d'un accord donné. La mise en place d'une nouvelle infrastructure dédiée aux publications s'organise autour de cinq éléments qui figurent presque systématiquement, selon un ordre variable, dans les annexes dédiées à la publication en accès ouvert.

1) l'indépendance éditoriale. Si les accords de notre corpus proposent des formules de publication en accès ouvert aux auteurs éligibles, aucun éditeur n'est cependant contraint à publier leurs textes: ces derniers doivent auparavant passer par le processus d'évaluation des revues. Autrement dit, seuls les articles acceptés pour publication peuvent bénéficier des modalités de publication en accès ouvert prévues dans un accord donné. Cette indépendance éditoriale des éditeurs est systématiquement rappelée sous une clause intitulée " editorial independance ». Elle commence généralement par la formule consacrée suivante : "Nothing herein shall oblige [publisher's name] to publish any article submitted to [publisher's name] by a Corresponding Author ". Ces dernières dispositions visent clairement à éviter un " devoir de publication » lié à une disponibilité monétaire et à contrer la critique selon laquelle les titres considérés seraient des "revues de complaisance », voire des « revues prédatrices ».

2) un ensemble de définitions sur l'éligibilité, qui concerne aussi bien les types de documents (les articles de recherche, les notes de lecture, mais plus rarement les communications ou le matériel pédagogique) que les auteurs, qui doivent toujours remplir deux conditions : d'une part, être enseignant, chercheur ou étudiant d'une université signataire de l'accord, de l'autre, être "corresponding author " (on trouve parfois la mention « submitting author »).

3) le processus d'identification des auteurs éligibles. Lorsqu'une institution signe un accord avec un éditeur, ses membres deviennent des tiers bénéficiant de l'accord et, en conséquence, ont accès aux modalités de publication en accès ouvert, tout comme 
ils ont accès en tant que lecteurs aux revues concernées par la partie abonnement. Par exemple, les membres des institutions rattachées au consortium VSNU peuvent collectivement publier 3600 articles en accès ouvert par an, dans le cadre de l'accord Elsevier-VSNU 2016. Cependant, lorsqu'un auteur souhaite publier en accès ouvert, il importe à l'éditeur de s'assurer de la réalité de son affiliation à une institution signataire de l'accord. Une trame relativement standard est repérable pour l'identification des auteurs éligibles : l'insistance sur la responsabilité de l'auteur et de l'institution en matière d'identification au cours d'un échange d'informations avec l'éditeur, notamment avec son adresse électronique ou un référent accès ouvert par institution ; l'utilisation d'une plateforme dédiée à l'identification; I'anticipation des problèmes d'identification et la description des modalités de rectification si un auteur effectivement éligible n'a pas été reconnu comme tel.

4) les modalités d'usage des fonds associés à la publication en accès ouvert. Les auteurs éligibles doivent s'astreindre à un certain nombre de règles d'usage, dont l'accord UK 2019 Springer/JISC fournit un exemple assez complet. Dans la partie sur l'accès ouvert (la « Part A » des accords Springer), on comprend que l'accord fonctionne sur la base d'un fonds d'APC (chaque année, il est possible de publier un certain volume financier d'articles en accès ouvert). Viennent ensuite des considérations d'usage du fonds: l'ordre de préséance ( first come first served basis », indépendamment de la somme payée par chaque institution), la date d'allocation (pour savoir à quelle année rattacher l'article), les règles en cas d'épuisement (Springer doit notifier le consortium avant épuisement du fond. Une fois épuisé, les institutions, voire les auteurs, peuvent ensuite payer à titre individuel pour l'ouverture des articles). D'autres accords précisent des conditions de report (ou de non report) des sommes ou du volume d'articles d'une année sur l'autre si le fonds annuel n'a pas été épuisé, ou encore des éléments sur l'ouverture rétrospective des articles publiés entre le début officiel de l'accord et la signature effective de l'accord par une institution.

5) la gestion de l'incertitude liée aux formules de publication en accès ouvert. L'accès ouvert tranche avec l'abonnement traditionnel, et les éditeurs et les consortiums ont mis en place au sein des accords, des modalités de traçabilité/surveillance. Plus précisément, l'éditeur est quasi-systématiquement tenu de fournir des informations régulières sur l'évolution de la publication en accès ouvert : production de rapports mensuels, bisannuels et annuels auprès du consortium et des institutions; mise à disposition de tableaux de bord; réunions annuelles avec le consortium, etc. Outre la dimension explicite des rapports de suivi, la gestion de l'incertitude se manifeste également dans la définition même de l'accès ouvert que proposent certains accords. 
Il en va ainsi des formules d'accès ouvert limitées par des quotas de publication ; ou des mécanismes financiers qui encadrent, voire limitent, l'évolution des montants.

\subsubsection{Les multiples formes de l'accès ouvert}

Pour importante qu'elle soit, la question du workflow ne dit rien de la nature des publications en accès ouvert qui circulent en son sein. Outre une définition minimale - un document en accès ouvert pour lequel la lecture est gratuite - les publications en accès ouvert renvoient dans les accords à une multitude d'options. Dans la figure 3.1, nous avons fait le choix de regrouper les huit options qui nous ont parues importantes dans les accords : le périmètre de publication, les licences, le degré d'obligation de la publication en accès ouvert, la voie verte, le modèle de paiement, les auteurs éligibles, le volume d'articles publiables, les sommes financières.

Figure 3.1 - Huit options

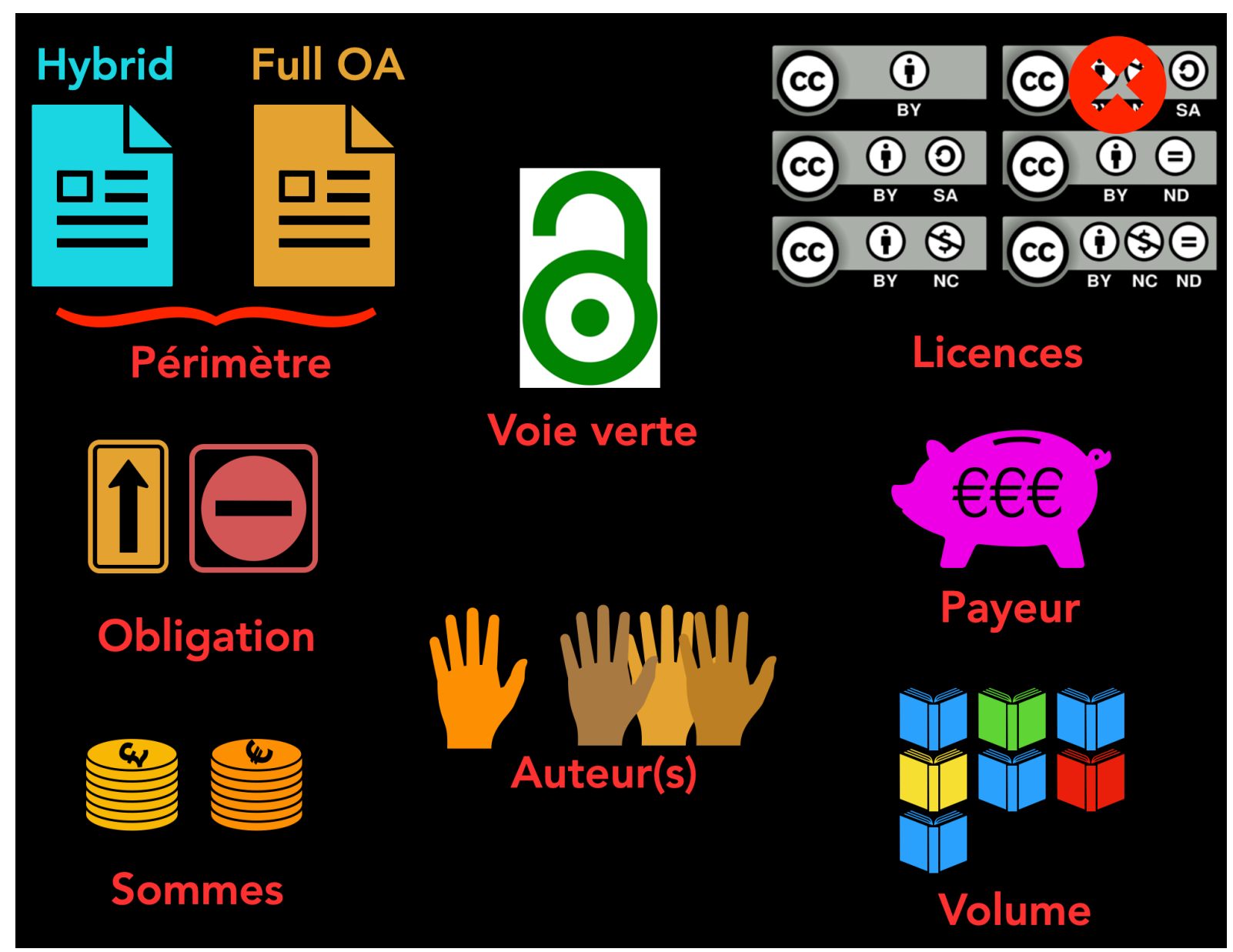

Pour chacune des huit options de la figure ci-dessus, on peut décliner différentes modalités. Passons-les en revue. 


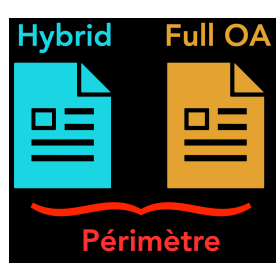

(c) (1) (c) 20 (c) (1) (2) (1) $\Theta$ (c) $($ i) $(\Theta)(D \Theta \Theta$

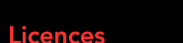

Le périmètre de publication. Certains accords réservent la publication en accès ouvert dans les revues hybrides seulement, d'autres dans les revues pleinement en accès ouvert seulement, ou encore dans les deux, voire dans des collections particulières. Quoi qu'il en soit, ce périmètre implique toujours de fournir une liste de revues éligibles à la publication en accès ouvert. Dans le cas des listes de revues hybrides, elles ne correspondent que rarement aux listes de revues concernées par l'objet « abonnement ».

Les licences. La réutilisation des publications en accès ouvert est systématiquement définie par une licence Creative Commons. Sur l'ensemble de notre corpus, toutes les licences sont déclinées à l'exception de la CC-BY-SA. Si certains accords imposent un seul type de licence Creative Commons pour l'ensemble des publications en accès ouvert, d'autres indiquent une obligation de licence CC différente suivant la revue concernée, sans doute en raison des choix des propriétaires de la revue et non de l'éditeur.

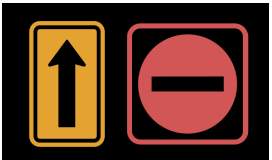

Obligation

Le degré d'obligation de la publication en accès ouvert. La très grande majorité des accords la proposent aux auteurs simplement comme une option (57 accords, 92\% du total). Cependant dans une petite minorité de cas, l'éditeur a l'obligation de proposer uniquement des licences en accès ouvert (5 accords, $8 \%$ du total). Cette clause contraint techniquement l'éditeur à publier les auteurs éligibles en accès ouvert, dans le cadre de l'accord.

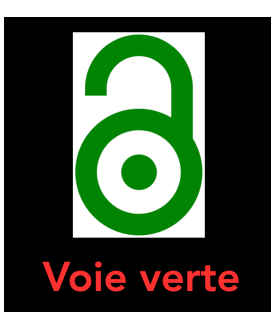

La voie verte. Pour la majorité des accords, la question du dépôt des contenus en archive ouverte hors du site de l'éditeur n'est pas abordée. On trouve cependant quelques exceptions qui proposent des formules vertes d'accès ouvert, que ce soit par le dépôt spécifique sur PubMed Central, par le rappel des politiques de l'éditeur en la matière, ou enfin par l'envoi de l'ensemble des publications vers une archive institutionnelle. 


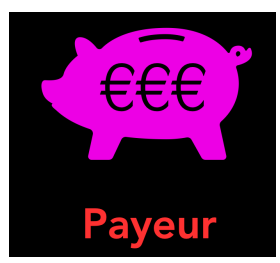

Le modèle de paiement. Nous reviendrons sur la notion de modèle de paiement (voir section 5). Retenons pour l'instant que la quasi-totalité des accords fonctionne sur un modèle de paiement unique et centralisé : le consortium paie directement l'éditeur pour le compte des institutions (et la répartition des frais entre les institutions au sein du consortium demeure obscure). Cependant, le cas singulier de UC/Springer propose une alternative, en promettant dans son "Memorandum of Understanding " de 2020 un modèle multi-payeurs original, qui répartit explicitement les frais entre la bibliothèque de l'University of California et les auteurs.

Les auteurs éligibles. Les accords montrent une convergence massive vers une solution unique : dans tous les documents consultés, l'auteur éligible n'est autre que le corresponding author, à partir du moment où il est affilié à une institution signataire. Néanmoins, certains accords Pure Publish récemment signés proposent d'élargir la définition de l'auteur éligible à l'ensemble des auteurs de l'article. Si cette modalité n'existe pas dans les accords transformants que nous avons lus, elle n'en constitue pas moins une modalité possible qui pourrait se développer dans les années à venir. En l'absence de littérature académique, des échanges avec des responsables de consortium nous indiquent que la prise en compte de tous les auteurs impliquerait un doublement du volume de production.

Le volume d'articles publiables. L'accord définit systématiquement un volume d'articles publiables par les auteurs, pris en charge dans le cadre de l'accord. Ce volume peut être un nombre fixe et prédéfini, un nombre encadré entre un minimum et un maximum, un volume limité par son coût total d'APC au prix catalogue, ou encore un volume illimité pour l'ensemble des auteurs affiliés aux institutions signataires de l'accord. Mis à part dans ce dernier cas, des règles de dépassement du volume sont généralement présentes. 


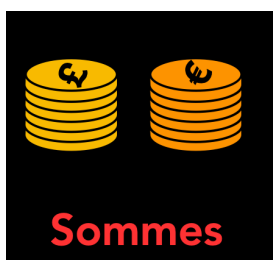

Les sommes financières. Comme indiqué précédemment, ces sommes peuvent être cachées ou enlevées de l'accord. Lorsqu'elles sont présentes, le montant total peut être lui aussi fixe, encadré par des bornes inférieure et/ou supérieure, ou potentiellement illimité. Nous verrons dans la section 5 que les modalités de ces deux dernières options (volume $d$ 'articles et sommes financières) entretiennent des relations étroites, impliquant de les penser ensemble.

Si ces huit éléments équipent considérablement la définition de la publication en accès ouvert, des combinaisons possibles entre eux viennent l'enrichir encore. Par exemple, l'accord suédois 2020 Elsevier/Bibsam propose une formule de publication en accès ouvert différente selon les bouquets de revues : illimitée et obligatoire sur la "Complete Freedom Collection", d'une part, limitée à 100 articles par an et non obligatoire sur la collection "Cell Press", d'autre part. Dans la même veine, l'accord allemand 2020 Springer/Projekt Deal accorde un volume encadré de publications dans les revues hybrides pour un montant encadré d'une part ; et une simple remise sur les APC, sans limitation de volume ni de montant, pour les revues en accès ouvert.

Les négociations et compromis entre consortiums et éditeurs produisent donc des agencements singuliers, conduisant à l'élaboration de schémas de publication à partir d'une gamme d'options qui peuvent à la fois être combinées de différentes manières, et modalisées selon le type de revues (hybride ou full OA). Cette gamme d'options donne lieu à des formules empiriques variées, dont certaines ont été rassemblées dans le tableau 3.2. Sans prétendre couvrir de manière exhaustive les formules rencontrées dans notre corpus, elles donnent un bon aperçu de leur multiplicité. Pour autant, elles ne dessinent pas des tendances générales. 
Tableau 3.2 - Exemples empiriques de formules d'accès ouvert

\begin{tabular}{|c|c|c|c|c|c|}
\hline $\begin{array}{c}\text { Cas } \\
\text { empirique }\end{array}$ & $\begin{array}{l}\text { Hungary } 2018 \\
\text { Springer/EISZ }\end{array}$ & $\begin{array}{c}\text { Netherlands } \\
2018 \\
\text { TandF/VSNU- } \\
\text { UKB }\end{array}$ & $\begin{array}{c}\text { Germany } \\
2019 \\
\text { Wiley/Projekt } \\
\text { Deal }\end{array}$ & $\begin{array}{c}\text { Netherlands } \\
2019 \\
\text { Emerlad/VSNU- } \\
\text { UKB }\end{array}$ & $\begin{array}{l}\text { Sweden } 2020 \\
\text { Elsevier/Bibsam }\end{array}$ \\
\hline $\begin{array}{l}\text { Périmètre } \\
\text { de } \\
\text { publication }\end{array}$ & Hybride & $\begin{array}{l}\text { Hybrides } \\
\text { seulement }\end{array}$ & $\begin{array}{l}\text { Hybrides sur } \\
\text { le fonds } \\
\text { d'APC ; } \\
\text { Discount sur } \\
\text { les full OA }\end{array}$ & $\begin{array}{l}\text { Hybrides et full } \\
\text { OA journals }\end{array}$ & $\begin{array}{l}\text { Hybrides et full } \\
\text { OA journals }\end{array}$ \\
\hline $\begin{array}{l}\text { Type de } \\
\text { licence }\end{array}$ & $\begin{array}{l}\text { CC-BY ou } \\
\text { CC-BY-NC }\end{array}$ & CC-BY-ND & CC-BY-ND & $\begin{array}{l}\text { CC-BY ou } \\
\text { CC-BY-NC }\end{array}$ & $\begin{array}{c}\text { CC-BY ou } \\
\text { CC-BY-NC-ND }\end{array}$ \\
\hline $\begin{array}{c}\text { Niveau } \\
\text { d'obligation }\end{array}$ & Optionnel & Optionnel & Optionnel & Optionnel & Obligatoire \\
\hline $\begin{array}{l}\text { Archives } \\
\text { ouvertes }\end{array}$ & Non & Non & Non & Oui & Non \\
\hline Temporalité & $\begin{array}{l}\text { Durée de } \\
\text { l'accord }\end{array}$ & Rétroactive & $\begin{array}{l}\text { Durée de } \\
\text { l'accord }\end{array}$ & $\begin{array}{l}\text { Durée de } \\
\text { l'accord }\end{array}$ & $\begin{array}{l}\text { Durée de } \\
\text { l'accord }\end{array}$ \\
\hline $\begin{array}{l}\text { Modèle de } \\
\text { paiement }\end{array}$ & Centralisé & Centralisé & Centralisé & Centralisé & Centralisé \\
\hline $\begin{array}{l}\text { Auteur } \\
\text { éligible }\end{array}$ & $\begin{array}{c}\text { Auteur } \\
\text { correspondant }\end{array}$ & $\begin{array}{c}\text { Auteur } \\
\text { correspondant }\end{array}$ & $\begin{array}{c}\text { Auteur } \\
\text { correspondant }\end{array}$ & $\begin{array}{c}\text { Auteur } \\
\text { correspondant }\end{array}$ & $\begin{array}{c}\text { Auteur } \\
\text { correspondant }\end{array}$ \\
\hline Volume & Encadré & Fixe & $\begin{array}{l}\text { Fixe sur les } \\
\text { hybrides; } \\
\text { Discount sur } \\
\text { les full OA }\end{array}$ & Fixe & Illimité \\
\hline Sommes & encadrés & fixés & Illimités & fixés & fixés \\
\hline
\end{tabular}




\section{Deux accords en un : les relations entre abonnement et publication}

Si l'abonnement et la publication en accès ouvert constituent les deux principaux éléments de la négociation entre les parties inscrites dans les accords, quelles relations entretiennent-ils entre eux ? Dans un premier temps, nous verrons que ces deux objets coexistent plus qu'ils n'interagissent. Selon les accords, l'importance relative de l'abonnement et de la publication en accès ouvert est variable, mais on observe toujours une partition nette entre le deux (4.1). La seule exception notable est d'ordre financier: l'abonnement et la publication en accès ouvert sont déterminants pour saisir les modalités de ventilation des sommes financières qui ont été négociées, et qui donnent par ailleurs le ton à la définition des types d'accords (4.2). Mais cette ventilation joue un strict rôle d'articulation. Les considérations financières ne remettent jamais en cause l'indépendance entre les deux objets.

\subsection{Importance relative de l'abonnement et de la publication en accès ouvert}

Dans les accords disponibles qui composent notre corpus, l'abonnement et la publication en accès ouvert ne se voient pas accorder la même importance : trois configurations possibles se distinguent nettement. Pour chacune, les deux objets entretiennent un rapport de coexistence, tout en demeurant totalement découplés : la présence de l'un n'influence pas le contenu de l'autre.

Dans la première configuration, l'abonnement est décisif (22 accords, soit 35\% du total). Cette prépondérance peut être affichée de manière explicite avec des phrases en début de document (dans le " corps de l'accord ", par opposition aux annexes), qui décrivent l'accord comme une formule d'abonnement, sans aucune mention de la publication en accès ouvert. L'accord Hungary 2019 RSC/EISZ l'énonce clairement en page 3, sous la clause « I. Subject of the contrat ", avec une phrase standard présente dans l'ensemble des accords signés par EISZ.

"The purpose of this Agreement is the subscription and access to Content (defined in Appendix 1) of the Supplier for the members of the Authorized Users described in Appendix 3 ». (Hungary 2019 RSC/EISZ, p. 3).

La primauté de l'abonnement est parfois manifeste dans l'organisation de certains documents. Ainsi, pour certains accords, le corps du document (par opposition aux annexes) 
ne mentionne que l'abonnement. C'est le cas du Finland 2018 Emerald/Finelib, qui en page 2, sous la clause " 2 . Agreement », évoque uniquement l'accès à des revues.

"The publisher agrees to grant the licencee [...] a non-exclusive and non-transferable right to access and use the licensed material and to allow authorized and walk-in users to access and use the licensed material throughout the terms of this agreement $"$. (Finland 2018 Emerald/Finelib, p. 2).

Dans d'autres cas de figure, la publication en accès ouvert se voit officiellement attribuer un statut limité, réservé à une annexe intitulée " pilot ». Enfin, dans certains cas, la publication en accès ouvert consiste en une simple réduction du prix catalogue des APC (discount). La publication en accès ouvert est ici un objet périphérique de l'accord, qui ne revêt aucune force obligatoire et même, parfois, n'implique pas les sommes en jeu dans l'accord, le paiement d'APC revenant aux auteurs.

La deuxième configuration possible est celle d'une présence équilibrée entre l'abonnement et la publication en accès ouvert (30 accords également, $48 \%$ du total). Cet équilibre est parfois explicité dans le corps de l'accord, où certains passages mentionnent expressément les deux objets, comme sur la première page du Sweden 2020 Elsevier/Bibsam :

"In consideration of the payment of a fee, Elsevier grants to the Subscriber the rights (1) to publish open access articles from Subscribers' authors in accordance with Schedule 3 herein; and (2) to access and use Elsevier products and services in accordance with the terms and conditions of this Agreement ". (Sweden 2020 Elsevier/Bibsam, p. 1).

De manière similaire, dans le corps de l'accord UK 2020 Wiley/JISC, en page 4, sous la clause "2. Agreement", on trouve la formule suivante :

"In consideration for the Publisher granting Eligible Authors the right to publish Eligible Articles open access in Hybrid and Gold Titles, and providing access to the Licensed Material and Additional Licensed Material (if licenced by Subscribing Institutions) to Authorised Users of Subscribing Institutions on the terms set forth in this Agreement ". (UK 2020 Wiley/JISC, p. 4).

L'équilibre est implicite lorsqu'aucun élément textuel ne met en avant l'un ou l'autre, et qu'ils occupent tous deux la même place dans l'accord, par exemple avec chacun une annexe dédiée.

Dans la troisième configuration, la publication en accès ouvert occupe une place de premier plan (10 accords, $16 \%$ du corpus). Cette primauté de l'accès ouvert sur l'abonnement est moins liée aux termes généraux de l'accord, qu'à la tournure explicite de certaines phrases. On peut distinguer ici deux éléments. D'une part, des paragraphes qui manifestent la volonté de s'éloigner du modèle de l'abonnement en faveur de l'accès ouvert, comme dans l'accord Austria 2019 Springer/KEMO : 
"The parties to this License Agreement recognize the important developments towards new Open Access publication methods and jointly agree that the present agreement represents a transitional business model whose aim is to provide a mechanism to shift to full open access in the near future ". (Austria 2019 Springer/KEMO, p. 1).

D'autre part, des affirmations sur un objectif de $100 \%$ de publications en accès ouvert dès le début de l'accord. C'est le cas de plusieurs accords signés par le consortium britannique JISC. Ainsi, dans UK 2020 European Respiratory Journal/JISC, on peut lire page 13 : " This pilot is designed to ensure 100\% OA for research articles for UK HE sector from $1^{\text {st }}$ Jan 2020 ». Outre ces mentions explicites sur la prépondérance de l'accès ouvert, évoquons un cas singulier en faveur de l'accès ouvert : I'accord UK 2020 ACM/JISC, dans lequel l'éditeur ACM propose une formule originale avec un modèle de transition vers l'accès ouverts en sept ans.

\subsection{La ventilation des sommes financières}

Qu'elles soient visibles ou non sur le document public, les sommes financières engagées par chaque accord sont sujettes à des ventilations variables entre l'abonnement et la publication en accès ouvert. Cette ventilation est importante, car elle préside souvent à la qualification du type d'accord, en lui attribuant un nom. Ainsi, les appellations aujourd'hui classiques - Offset Agreement, Read \& Publish, Publish \& Read-véhiculent une définition essentiellement financière des accords transformants. Le mode de ventilation entre l'abonnement et la publication peut être considéré comme une convention comptable, un choix concernant la représentation financière des accords.

Évidemment, une telle convention a des conséquences importantes sur les parties et leurs capacités d'action respectives. Par exemple, le fait de ventiler le montant uniquement sur la publication en accès ouvert occasionne des réflexions complexes sur la répartition des frais entre les institutions du consortium. Autre exemple : si l'abonnement ne devient plus qu'une fraction du coût pour le consortium (en lieu et place du total), comment ce coût serat-il évalué dans l'accord, et dans les accords suivants?

La lecture approfondie du corpus nous a permis de distinguer cinq modes de ventilation financière, soit cinq types d'accords transformants du point de vue financier. Alors que les trois premiers constituent des formes aujourd'hui connues et documentées, les deux derniers sont directement tirés de nos analyses.

1) L'Offset Agreement ( 1 accord, $2 \%$ du corpus), employé ici au sens strict, se réfère à un mécanisme de compensation : dans un accord offset, les frais d'abonnement ont reversés intégralement (ou presque) pour la publication en accès ouvert. Autrement dit, tous les frais engagés pour la publication sont déduits du montant global de l'abonnement. 
2) Le Publish \& Read (25 accords, $40 \%$ du total). Ici, aucune ventilation n'a cours à proprement parler entre l'abonnement et la publication, mais les deux sont englobés dans un seul montant. Cette absence de distinction est parfois thématisée, comme dans les accords DEAL qui évoque, au lieu des APC, un PAR Fee (Publish \& Read), indiquant bien que c'est la combinaison des services qui est ainsi payée.

3) Le Read \& Publish (33 accords, 53\% du corpus) constitue une catégorie classique des "transformative agreements». II propose deux montants distincts, I'un pour l'abonnement à des revues, l'autre pour la publication en accès ouvert.

4) Le Read \& Free Articles ( 1 accord, $2 \%$ du corpus) est un type d'accord caractérisé par un seul montant. Celui-ci combine l'abonnement à un faible volume de publications en accès ouvert. On parle habituellement de " publish for free " dans la mesure où le volume de publications en accès ouvert est tellement réduit qu'il peut être considéré comme un service gratuit, offert par l'éditeur en plus de l'abonnement. La définition d'un volume faible est aisée pour les cas extrêmes, comme Czech Republic 2019 IEEE/Czech ELIB qui ne propose que 4 articles en accès ouvert. En revanche, la difficulté réside dans la délimitation entre un volume faible et un volume significatif. Afin d'établir un seuil pour cette catégorie, nous proposons le critère suivant: en multipliant le volume par un prix d'APC moyen, on peut évaluer un montant de la publication. Si celui-ci est inférieur à $20 \%$ du montant total, il est considéré comme non significatif.

5) Le Read \& Discount ( 2 accords, 3\% du corpus) est un accord classique, avec une somme pour l'abonnement auquel vient s'ajouter un service de publication en accès ouvert à prix réduit par rapport au prix catalogue : un discount sur les APC individuels qui varie entre $-15 \%$ et $-70 \%$. Le discount a ceci de particulier que les montant associés aux deux objets (abonnement et publication en accès ouvert) n'ont pas le même statut. Quand le montant de l'abonnement est nécessairement endossé par le consortium, le montant du discount est en revanche potentiel, au sens où il ne sera payé que si et seulement si un auteur éligible choisit de publier un article en accès ouvert. Certains accords du corpus (7, soit $12 \%$ du total) proposent une formule discount, généralement sur les revues pleinement en accès ouvert, en plus d'une autre formule comprenant de l'accès ouvert (voir ci-dessous).

Le tableau 4.1 propose une distribution de ces différentes formes de ventilation financière au sein de notre corpus. 
Tableau 4.1 - Distribution des formes de ventilation financière

\begin{tabular}{|c|c|c|}
\hline Formes de ventilation & $\mathbf{N}$ & $\%$ \\
\hline Offset Agreement & 1 & 2 \\
\hline Publish \& Read & 25 & 40 \\
\hline Read \& Publish & 33 & 53 \\
\hline Read \& Free Articles & 1 & 2 \\
\hline Read \& Discount & 2 & 3 \\
\hline Total & 62 & 100 \\
\hline
\end{tabular}

En conclusion, insistons sur le fait que l'abonnement et la publication en accès ouvert constituent le socle de la ventilation des sommes financières, ce qui ne les empêche pas de rester parfaitement hermétiques l'un vis-à-vis de l'autre. Leur coexistence au sein des accords, va également de pair avec un strict découplage. Comme on l'a vu, l'abonnement est systématiquement configuré dans un monde fermé, soumis à paiement qui déclenche des séries d'identifications des circulations légitimes tant du contenu informationnel que des usagers. II insiste notamment sur les interdictions de réutilisation ou même de copie des articles scientifiques. À l'opposé, la publication en accès ouvert est attachée à un monde plus ouvert, puisque l'accès au contenu est gratuit, qui induit des préoccupations de gestion du workflow et des modalités d'accessibilité.

De plus, les différents éléments constitutifs de ces objets contractuels ne sont pas couplés : d'un côté, les lecteurs sont constitués de l'ensemble des membres des institutions abonnées, de l'autre, seuls les auteurs correspondants sont concernés ; les listes de revues accessibles et celles où on peut publier en accès ouvert sont distinctes ; les workflows ont des objectifs et des organisations matérielles totalement différents, etc. Bien qu'ils se trouvent négociés au sein d'un même accord, et qu'ils soient plus ou moins articulés en fonction des formules financières, l'abonnement et la publication en accès ouvert sont donc toujours traités séparément. 


\section{Section 5.}

\section{Les arrangements financiers de la publication en accès ouvert}

Si la section précédente a permis d'introduire la question de la ventilation des sommes entre l'abonnement et la publication, nous proposons ici d'entrer dans le détail des arrangements financiers associés à la publication en accès ouvert : comment fonctionnent-ils ? Comment sont-ils calculés ? Qui paie concrètement? La difficulté d'appréhension des arrangements financiers réside dans le fait qu'ils combinent conjointement deux préoccupations: des sommes financières et des volumes de publications.

Dans un premier temps, nous proposerons une catégorisation de ces mécanismes en fonction de la manière dont ils distribuent les incertitudes sur les sommes et les volumes de publication (5.1). Nous verrons ensuite les formules concrètes qui permettent à chaque mécanisme d'aboutir à un montant total, soit le coût pour le consortium et le revenu pour l'éditeur (5.2). La troisième partie s'intéressera aux modalités d'évolution de ce montant total (5.3). Enfin, nous évoquerons le problème de la répartition des sommes entre les payeurs (5.4).

\subsection{De la prévisibilité à l'incertitude : une catégorisation des arrangements financiers}

L'abonnement se caractérise toujours par un arrangement financier unique et une parfaite prévisibilité des montants en jeu : en signant un accord, un consortium s'engage à verser à l'éditeur une somme d'argent fixée à l'avance, qui ouvre des droits d'accès à des revues préalablement identifiées et d'usages encadrés, pour un volume d'usagers lui aussi défini. L'accès ouvert instaure une situation assez différente, pour au moins trois raisons. Tout d'abord, les sommes financières ne sont plus attachées à des revues accessibles pour des usagers, mais à des volumes de publications d'auteurs des institutions concernées. Ensuite, on passe d'un arrangement financier unique et vecteur de prévisibilité à plusieurs montages qui distribuent l'incertitude et potentiellement le risque de manière variable entre les sommes et les volumes d'articles. Enfin, plusieurs arrangements financiers propres à la publication en accès ouvert occasionnent des formes de débordement, soit des situations à la marge, où la relation financière entre les parties change. Autrement dit, il existe des accords avec un arrangement financier principal, mais qui peut basculer dans un aménagement secondaire 
sous certaines conditions. En nous attachant au fil conducteur de la distribution de l'incertitude, nous avons regroupé les accords de notre corpus autour de quatre catégories d'arrangements financiers: somme fixe/volume fixe (5.1.1), corridor (5.1.2), pay as you publish (5.1.3), et somme fixe/volume illimité (5.1.4). Insistons ici sur le fait qu'il s'agit avant tout d'un regroupement conventionnel commode, et non l'expression d'une évolution historique nécessaire.

Le tableau 5.1 montre la distribution de ces quatre arrangements financiers au sein des accords. Pour chacun d'eux, nous préciserons la répartition du risque qui pèse sur la somme en jeu, l'incertitude qui pèse sur le volume de publications, ainsi que les modalités éventuelles de débordement.

Tableau 5.1 - Distribution des arrangements financiers

\begin{tabular}{|c|c|c|}
\hline Arrangements financiers & $\mathbf{N}$ & $\%$ \\
\hline Somme fixe/volume fixe & 38 & $61 \%$ \\
\hline Corridor & 8 & $13 \%$ \\
\hline Pay as you publish & 6 & $10 \%$ \\
\hline Somme fixe/volume illimité & 10 & $16 \%$ \\
\hline Total & 62 & $100 \%$ \\
\hline
\end{tabular}

\subsubsection{Somme fixe/volume fixe}

L'arrangement somme fixe/volume fixe se retrouve dans la majorité des accords étudiés (38 accords, $61 \%$ du corpus). Cette configuration majoritaire consiste à fixer les volumes de publications et les montants. C'est le cas d'un accord comme Hungary 2019 Springer/EISZ, avec 520 articles pour $1190800 €$ sur une année. Cet arrangement financier reconduit l'enjeu de prévisibilité qui caractérise l'abonnement. Ici, ni le consortium ni l'éditeur ne prennent de risques : les frais du consortium, les recettes de l'éditeur, et les volumes de publication sont connus à l'avance.

Cependant, contrairement à l'abonnement, l'arrangement somme fixe/volume fixe attaché à la publication en accès ouvert admet une forme de débordement. En effet, une fois le volume d'articles épuisé (dans l'exemple cité, les 520 articles), la nature financière de la relation entre les parties change. On bascule sur un arrangement financier secondaire fondé sur le retour au paiement d'APC pour chaque article individuel. Autrement dit, une fois le volume d'articles épuisé, le consortium cesse de payer pour la publication en accès ouvert, mais les institutions et les auteurs individuels ont la possibilité de le faire sur leurs deniers propres. L'accord norvégien entre Wiley et Unit datant de 2019 apporte une subtilité : si l'arrangement principal est tout à fait classique, l'aménagement secondaire constitue un 
retour à I'APC individuel avec une réduction. Ainsi, lorsque la limite de publication a été atteinte, les institutions ou les auteurs ont la possibilité de payer pour publier en accès ouvert à un prix réduit (-20\%).

Signalons enfin le cas particulier des accords Read \& Discount, pour lesquels le volume d'article est nul, puisque l'ensemble du montant est alloué à l'abonnement, et que la publication en accès ouvert est un objet annexe, à prix réduit, à la charge des auteurs ou des institutions. Si la somme est fixe, il n'y a pas de volume, et aucun débordement possible.

\subsubsection{Corridor}

Le deuxième arrangement financier est un aménagement du premier. Le corridor est présent dans 8 accords ( $13 \%$ du total). Le principe de base est toujours le même : un montant fixé pour un volume de publications donné. À cela, l'accord adjoint un corridor de publications qui vient encadrer le volume de publications de base. Par exemple, dans l'accord Suédois 2019 Springer/Bibsam, le volume de base pour 2019 est de 1578 articles, délimité par une borne basse (1 449 articles) et une borne haute (1 657 articles).

Le corridor peut jouer plusieurs rôles. D'une part, il définit un volume au sein duquel la somme financière est inchangée. Ici, le débordement intervient à la baisse : si le volume minimal d'articles n'est pas atteint, le consortium obtient un remboursement partiel - comme à la hausse ; si le volume maximum est atteint, on bascule vers l'APC individuel financé par les institutions ou les auteurs. Par exemple, l'accord Norway 2020 Springer/Unit définit chaque année un corridor (593-651 en 2020 ; 608-668 en 2021 ; 623-684 en 2022) qui limite le montant à la hausse et oblige l'éditeur à rembourser pour les articles non publiés si la limite basse n'est pas atteinte. Cette forme de corridor fait peser un risque sur l'éditeur, qui ne gagnera pas plus que la somme convenue, mais peut être amené à rembourser le consortium. Il existe des cas singuliers où le risque est réparti, comme dans l'accord Austria 2019 Springer/KEMÖ, où le remboursement de l'éditeur ne peut dépasser 60000 euros, tandis que le dépassement de la borne haute est autorisé dans la limite de 60000 euros.

D'autre part, le corridor définit une zone au sein de laquelle la somme et le volume de publications en accès ouvert peuvent varier, sans jamais dépasser les deux bornes. C'est le cas unique du Germany 2020 Springer/Projekt Deal. Nous présenterons dans la partie 5.2. le détail de la formule financière. Pour le moment, précisons que l'accord propose une fourchette de publications, avec une borne haute et une borne basse, dont l'amplitude augmente avec le temps. Le montant varie en fonction du volume effectif de publications, mais toujours dans les limites de la fourchette (figure 5.1.). Ce type de corridor induit deux types de débordement. À la baisse, puisqu'en dessous d'un certain volume de publications, on bascule sur un montant fixe correspondant à celui de la borne basse. À la hausse, une fois la borne haute atteinte, le consortium arrête de payer et les auteurs peuvent publier en accès ouvert de manière illimitée, sans occasionner de frais supplémentaires. C'est ni plus ni moins la définition de la 
quatrième et dernière catégorie d'arrangement financier que nous aborderons plus loin, et que nous appelons somme fixe/volume illimité.

Figure 5.1 - Les coûts de publication du Germany 2020 Springer/DEAL

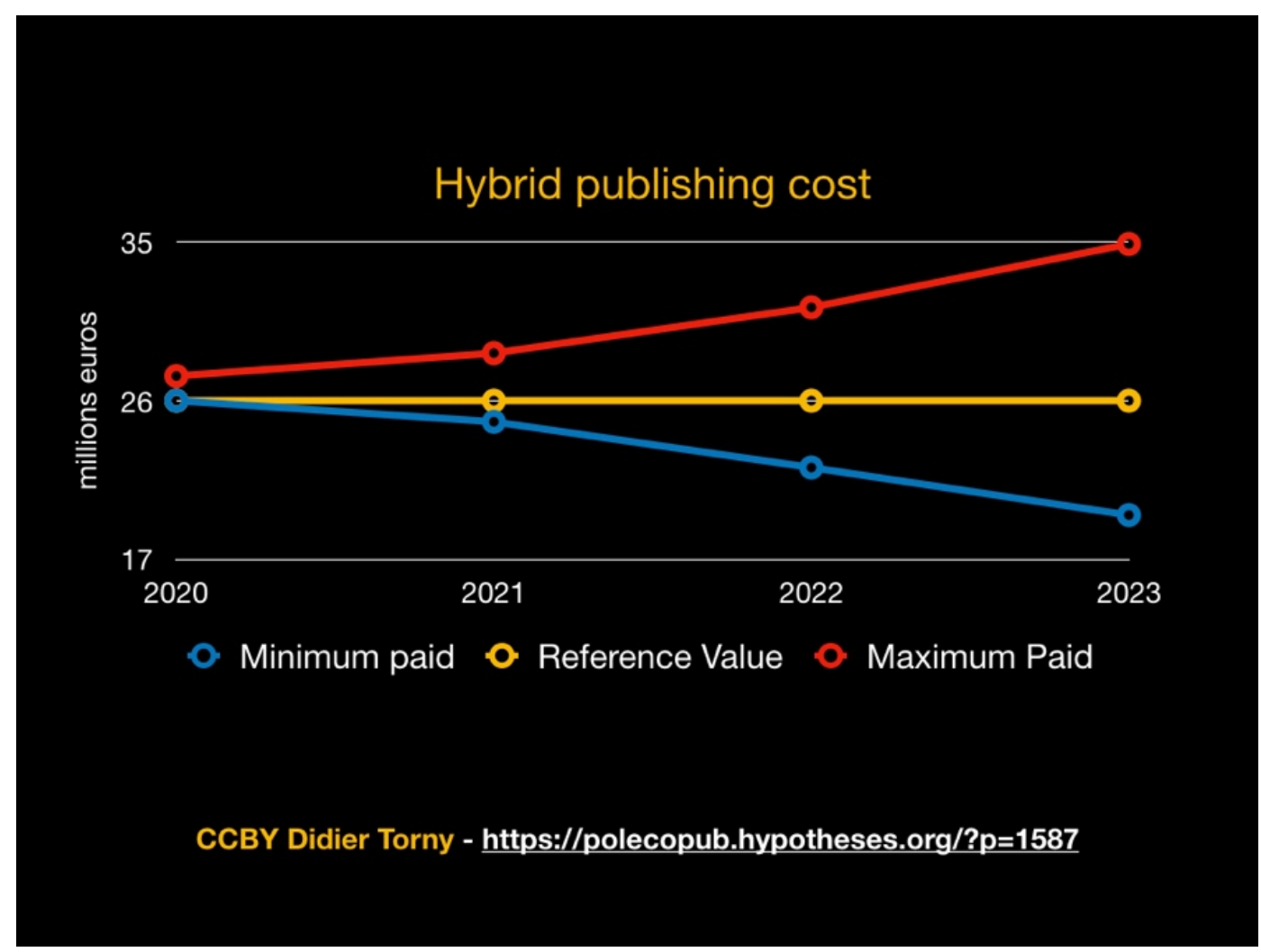

Source : https://polecopub.hypotheses.org/1587

\subsubsection{Pay as you publish}

L'arrangement financier Pay as you publish caractérise un alignement du montant sur le volume effectif de publication en accès ouvert ( 6 accords, $10 \%$ du corpus). Ici, plus une institution publie, plus elle paie. On retrouve ce type d'arrangement dans plusieurs accords signés avec le consortium britannique JISC (UK 2020 Compagny of Biologists/JISC, UK 2020 Portland Press/JISC, et UK 2020 European Respiratory Journal/JISC). À la différence des arrangements précédents, l'incertitude est totale, tant sur le montant que sur le volume de publication. Ici, le risque financier est donc important pour les deux parties. En effet, si la publication en accès ouvert est importante, le consortium verra son coût s'envoler, alors que si elle est faible, c'est l'éditeur qui pâtira d'un manque à gagner.

Parmi les accords attachés à un arrangement Pay as you publish, la majorité n'inclut aucune forme de débordement. Autrement dit, les accords ne basculent jamais dans un arrangement secondaire où la relation financière entre les parties se trouve transformée. II existe cependant une exception notable, à savoir, l'accord UK 2020 ACM/JISC. Fondé sur le modèle original de l'éditeur ACM open pour la transition vers l'accès ouvert en sept ans, cet accord propose un « tier-based system model » où les institutions sont classées au sein de 10 
échelons selon leur volume de publications. Plus une institution publie, plus elle se trouve dans les tranches hautes, plus elle paie ; sachant qu'il existe une limite haute au-delà de laquelle le consortium cesse de payer. Nous sommes donc bien dans un arrangement Pay as you publish où le risque financier pèse sur le consortium. Cependant, une fois l'échelon le plus haut atteint, l'accord bascule dans un arrangement secondaire de type somme fixe/volume illimité. On retrouve dans l'accord UK 2020 ACM/JISC le même type de débordement que dans le Germany 2020 Springer/Projekt Deal évoqué précédemment.

\subsubsection{Somme fixe/volume illimité}

Présent dans 10 accords (16\% du corpus), l'arrangement financier somme fixe/volume illimité opère un retour à une somme totale fixée, mais permet aux auteurs éligibles de publier sans limite en accès ouvert. À titre d'exemple, l'accord Netherlands 2020 Elsevier/VSNU-UKB propose une somme globale fixe que le consortium paiera de toute façon (14 $660000 €$ en 2020 ; puis $16460000 €$ pour chacune des quatre années suivantes). Ce montant fixé à l'avance permet aux auteurs de publier un nombre illimité d'articles en accès ouvert. On retrouve ainsi le niveau de prévisibilité financière du premier arrangement financier décrit plus haut (somme fixe/volume fixe), mais également celui de l'abonnement. Cependant, la répartition du risque est particulière, et se mesure en termes de coût d'opportunité. Pour le consortium, le risque consiste à payer un montant important pour un volume de publications inconnu qui sera potentiellement faible - le prix de l'article individuel serait alors très élevé. Pour l'éditeur, le risque est inversé, puisque plus les institutions publient, plus le prix individuel de l'article baisse. Logiquement, l'arrangement somme fixe/volume illimité n'admet aucune modalité de débordement : le montant est fixé indépendamment du volume effectif de publications.

\subsection{Les formules financières de la publication en accès ouvert}

Avec ces quatre arrangements financiers généraux associés à la publication en accès ouvert, nous disposons désormais d'une représentation de l'espace des possibles concernant la variation du montant financier et du volume d'articles dans les accords. Mais comment les sommes de la publication en accès ouvert sont-elles effectivement fixées ? Selon quelles formules concrètes les arrangements financiers se déploient-ils pour attribuer un montant à la publication ? C'est à ces questions que nous répondrons dans cette partie.

Premier élément majeur : la majorité des formules financières n'est tout simplement pas connue, car non mentionnée dans les clauses contractuelles (40 accords, $65 \%$ du total). Pour le reste, au fil de nos lectures, nous avons pu identifier cinq types de formules : la réduction d'APC, la formule " volume x prix individuel », l'Offset, le total des APC des années précédentes, et certains cas singuliers difficiles à classer. Leur distribution au sein des accords est résumée dans le tableau 5.2 : 
Tableau 5.2 - Distribution des formules financières

\begin{tabular}{|l|c|c|}
\hline \multicolumn{1}{|c|}{ Formules financières } & N & $\%$ \\
\hline Réduction APC & 2 & $3 \%$ \\
\hline Cas singulier & 7 & $11 \%$ \\
\hline Non & 40 & $65 \%$ \\
\hline Vol article*APC indiv & 9 & $15 \%$ \\
\hline Offset & 1 & $2 \%$ \\
\hline Moyenne APC années passées & 3 & $5 \%$ \\
\hline
\end{tabular}

Afin de présenter les formules financières, nous proposons de repartir des arrangements décrits précédemment. Dans ce qui suit, nous évoquerons successivement les formules attachées à l'arrangement somme fixe/volume fixe (5.2.1), au Corridor (5.2.2), au Pay as you publish (5.2.3), et enfin au somme fixe/volume illimité (5.2.4).

\subsubsection{Les formules financières associées à Somme fixe/volume illimité}

L'arrangement Somme fixe/volume fixe prévoit à l'avance le montant en jeu et le volume de publications autorisé. Les formules financières associées à cet arrangement sont inconnues pour une large majorité ( 29 accords, $76 \%$ des accords arborant ce mécanisme). Cela étant dit, on peut néanmoins repérer des formules récurrentes.

La plus répandue renvoie au dispositif classique du fonds d'APC, et consiste à établir un volume d'articles à publier ainsi qu'un prix à l'unité. Le montant de la publication en accès ouvert tient alors dans le produit du volume total par le prix à l'unité. On retrouve principalement cette formule dans les accords signés par Springer, comme dans le cas déjà cité de l'accord hongrois 2019 Springer/EISZ, avec 520 articles à 2290 euros, pour un coût de l'accès ouvert qui s'élève à 1190800 euros. Il en est de même pour l'accord britannique 2019 Springer/JISC, qui dure trois ans : celui-ci fixe chaque année un volume d'articles (4 808, 4 934, 5 127), un prix à l'unité (2 244€, $2289 €$ et $2335 €$ ), dont le produit donne le montant total de la publication (10 $223664 €, 10669029 €$ et $11100590 €$ ).

L'arrangement Somme fixe/volume fixe abrite deux autres formules plus marginales. La réduction sur les APC d'une part, caractéristique des accords " Read and Discount ». Ici, le montant porte entièrement sur l'abonnement ; tandis que le prix individuel de la publication, à prix réduit, est à la charge de l'institution ou de l'auteur individuel (comme dans le cas de I'accord français 2019 Elsevier/Couperin avec une réduction de $25 \%$ sur le prix catalogue des $A P C)$. D'autre part, l'Offset, soit un mécanisme compensatoire où les frais déboursés pour 
I'abonnement sont utilisés pour publier en accès ouvert. Le seul accord de notre corpus qui relève de ce cas de figure est le néerlandais 2016 Walter de Gruyter/VSNU-UKB. Ici, 90\% (soit presque la totalité) des frais d'abonnement sont utilisés pour la publication en accès ouvert. Ainsi, sur les 166 604€ réglés à l'éditeur, 149 943€ sont attribués à la publication d'articles en accès ouvert.

Tableau 5.3 - Exemples de formules financières associées à Somme fixe/volume fixe

\begin{tabular}{|l|l|l|}
\hline \multicolumn{1}{|c|}{ Nom de l'accord } & \multicolumn{1}{|c|}{ Formule financière } & \multicolumn{1}{c|}{ Calcul } \\
\hline Hungary 2019 Springer/EISZ & Volume x prix à l'unité & 520 articles x 2 290€=1190 800€ \\
\hline UK 2019 Springer/JISC & Volume x prix à l'unité & $\begin{array}{l}4808 \text { articles } \times 2244 €= \\
10223664 €(p o u r ~ 2019)\end{array}$ \\
\hline France 2019 Elsevier/Couperin & Discount & $-25 \%$ sur les APC \\
\hline $\begin{array}{l}\text { Netherlands 2016 Walter de } \\
\text { Gruyter/VSNU-UKB }\end{array}$ & Offset & $\begin{array}{l}\text { 90\% de l'abonnement finance la } \\
\text { publication en accès ouvert }\end{array}$ \\
\hline
\end{tabular}

\subsubsection{Les formules financières associées au Corridor}

L'arrangement Corridor fixe une somme et un volume de publications, tout en encadrant ce volume par une borne haute et une borne basse. Plus haut, nous avons vu que le corridor pouvait jouer deux rôles.

D'une part stabiliser une somme au sein des deux bornes de volume, et prévoir des arrangements secondaires de débordement à la baisse (remboursement partiel) et à la hausse (retour à l'APC individuel). Dans ce cas de figure, la formule financière n'est autre que celle déjà évoquée du fonds d'APC, à savoir "volume x APC individuel », comme pour le Norway 2020 Springer/Unit, qui pose chaque année un corridor (593 651 en 2020 ; 608668 en 2021 ; 623684 en 2022). Ici, le calcul de la somme annuelle correspond au produit d'un volume d'articles de base qui se situe entre les bornes du corridor (622 en 2020, 638 en 2021, 654 en 2022 ) et un prix individuel ( $2289 €$ pour 2020, $2235 €$ pour 2021, $2281 €$ pour 2022). On arrive à un montant total de $1423458 €$ pour 2020 ; $1489730 €$ en 2021 ; et enfin $1557174 €$ pour 2022.

D'autre part, le corridor peut définir une zone de variation du montant en fonction du volume de publications, sans jamais dépasser les limites hautes et basses. Revenons ici sur le fonctionnement du cas unique de l'accord Germany 2020 Springer/Projekt Deal, qui propose une formule "volume x APC individuel " singulière. On commence par évaluer un nombre d'articles à publier (9 500) auxquels on accorde un prix de publication à l'unité (2 750 euros). De là, on calcule une valeur de référence: 9500 (articles) $\times 2750$ (prix unitaire) = 26125000 euros. Ensuite, le coût peut évoluer en fonction du nombre effectif de 
publications, au sein d'une fourchette autour de la valeur de référence. Plus le temps passe, plus l'amplitude de la fourchette augmente (+5\%; $95 \% /+10 \% ; 85 \% /+20 \% ; 75 \%+30 \%)$. Plus le temps passe, plus les coûts peuvent varier à la hausse ou à la baisse en fonction du nombre effectif de publications, mais toujours dans les limites de la fourchette (voir la figure 5.1. plus haut).

Tableau 5.4 - Exemples de formules financières associées au Corridor

\begin{tabular}{|l|l|l|}
\hline \multicolumn{1}{|c|}{ Nom de l'accord } & \multicolumn{1}{c|}{ Formule financière } & \multicolumn{1}{c|}{ Calcul } \\
\hline Norway 2020 Springer/Unit & Volume x APC individuel & $\begin{array}{l}622 \text { articles } \times 2289 €=1423458 € \text { pour } \\
\text { l'année 2020. }\end{array}$ \\
\hline $\begin{array}{l}\text { Germany 2020 } \\
\text { Springer/Projekt Deal }\end{array}$ & Cas singulier & $\begin{array}{l}9500 \text { articles } \times 2750 €=26125000 €, \\
\text { puis variation du montant au sein du } \\
\text { corridor autour de la valeur de référence } \\
(+5 \% ; 95 \% /+10 \% ; 85 \% /+20 \% ; \\
75 \% /+30 \%)\end{array}$ \\
\hline
\end{tabular}

Pour terminer, signalons que parmi les accords avec Corridor, un seul (13\%) ne fait pas mention de la formule financière sur laquelle il s'appuie.

\subsubsection{Les formules financières associées au Pay as you publish}

L'arrangement Pay as you publish indèxe directement la somme payée par le consortium à l'éditeur sur le volume effectif de publications en accès ouvert. Bien que peu dans notre corpus ( 6 accords, $10 \%$ du total), leur formule financière est systématiquement connue. La plus récurrente est la moyenne du montant des APC des années précédentes. Plus précisément, cela consiste à calculer les frais moyens d’APC engagés les années précédentes, puis à augmenter cette somme d'un certain pourcentage pour l'année en cours. C'est ce que I'on retrouve dans trois accords britanniques : le montant de l'accès ouvert pour UK 2020 Compagny of Biologists/JISC renvoie au montant moyen des APC sur la période 2016-2018 majoré de 2\%. Même idée pour UK 2020 Portland Press/JISC et UK 2020 European Respiratory Journal/JISC, quoique le montant moyen porte sur la période 2017-2018.

II existe cependant des cas singuliers. Premier exemple, l'accord UK 2020 ACM/JISC, signé pour 3 ans, s'inscrit dans un modèle assumé par l'éditeur de transition vers l'accès ouvert. Ici, le "tier-based system model " échelonne les institutions en fonction du nombre moyen de publications sur les trois dernières années. Plus une institution publie, plus elle se trouve dans les tranches hautes, plus elle paie (entre $8000 \$$ et $30000 \$$ pour 2020 ; entre 
$8000 \$$ et $60000 \$$ pour 2021 ; entre $8000 \$$ et $100000 \$$ pour 2022) ; jusqu'à arriver au dernier échelon, où la publication en accès ouvert devient illimitée.

Deuxième exemple, l'accord néerlandais 2019 IWA/Delft \& Wageningen University, également de trois ans, propose une formule complexe pour l'année 2019: [moyenne annuelle du montant de souscription des années 2015-16-17] / $2+$ [somme des APC que les institutions auraient payé si les articles avaient été publiés en $\mathrm{OA}] / 2$ avec une hausse de $1 \%$. Pour les années suivantes, le coût total est une modulation du coût de 2019 en fonction d'un taux de variation des articles publiés. Par exemple, pour 2020, le taux est calculé comme suit : [Articles publiés sur 2017-2019] / [articles publiés sur 2016-2018].

Tableau 5.5 - Exemples de formules financières associées au Pay as you publish

\begin{tabular}{|l|l|c|}
\hline \multicolumn{1}{|c|}{ Nom de l'accord } & \multicolumn{1}{|c|}{ Formule financière } & Calcul \\
\hline $\begin{array}{l}\text { UK 2020 Compagny of } \\
\text { Biologists/JISC }\end{array}$ & APC moyen sur 2016-2018 +2\% d'augmentation & $?$ \\
\hline $\begin{array}{l}\text { UK 2020 Portland } \\
\text { Press/JISC }\end{array}$ & APC moyen sur 2017-2018 +2\% d'augmentation & $?$ \\
\hline $\begin{array}{l}\text { UK 2020 European } \\
\text { Respiratory Journal/JISC }\end{array}$ & APC moyen sur 2017-2018 +2\% d'augmentation & $?$ \\
\hline UK 2020 ACM/JISC & $\begin{array}{l}\text { Volume moyen des trois dernières années, associé à des } \\
\text { échelons en fonction du montant en jeu }\end{array}$ & $?$ \\
\hline $\begin{array}{l}\text { Netherlands 2019 IWA/Delft } \\
\text { \& Wageningen University }\end{array}$ & $\begin{array}{l}\text { En 2019 : moyenne annuelle du montant de souscription } \\
\text { des années 2015-16-17] } / 2+[\text { somme des APC que les } \\
\text { institutions auraient payé si les articles avaient été publiés } \\
\text { en OA] /2 avec une hausse de 1\%. }\end{array}$ & $?$ \\
\hline
\end{tabular}

\subsubsection{Les formules financières associées au Somme fixe/volume illimité}

Sur l'ensemble du corpus étudié, seulement 10 accords (16\% du total) proposent un arrangement financier où la somme est fixée par les parties, mais où le volume de publications en accès ouvert est illimité, pour le périmètre des revues considérées. Effet direct de la variabilité des politiques de transparence des consortiums et des éditeurs, ces dix accords ne dévoilent jamais les formules permettant de calculer les montants en jeu. En se fondant uniquement sur la lecture des documents, il est dès lors impossible de savoir sur quelles formules financières concrètes ils reposent.

\section{3. Évolutions du montant total}

L'enjeu de la maîtrise des coûts pour les consortiums est une constante depuis au moins l'émergence des Big Deals. Aussi les accords transformants sont-ils souvent présentés, à la 
manière d'OA2020, comme des moyens concrets de limiter ces montants, notamment par des logiques de compensation, que ce soit par un Offset direct ou d'autres moyens. De plus, les principes mis en avant par les associations et consortiums de bibliothèques pour la négociation des accords (voir section 6) incluent presque toujours l'idée de " cost neutrality ", soit la fin des hausses du montant de la facturation par l'éditeur. Et c'est la raison qui guide les modalités de ventilation observées dans la section $4:$ c'est certes une convention, mais une convention essentielle au moins pour les consortiums afin de soutenir une logique de basculement du financement.

On retrouve cet aspect conventionnel dans la distribution financière à l'intérieur du contrat lui-même : comme on l'a vu en section 2 , la très grande majorité des accords sont pluriannuels. Cependant, en dépit de cette durée, l'ensemble des éléments financiers est systématiquement indiqué année par année - bien entendu dans le montant public. On peut avancer plusieurs explications à cela : budgets publics et parapublics annuels, possibilités d'opt-out et d'opt-in du marché, calculs itératifs d'une année à l'autre dans certaines formules financières. Cette annualisation a pour première conséquence de permettre une évolution des montants à l'intérieur même d'un contrat. Parmi les données brutes du fichier Available_TA_TotalSums fourni avec ce rapport, focalisons-nous sur les accords signés par l'éditeur Elsevier.

Certains voient leur montant augmenter comme le VSNU 2016 néerlandais, le Unit 2019 norvégien (avec des volumes de publication en accès ouvert qui augmentent) ; le Bibsam 2020 suédois avec de la publication illimitée. D'autres ont globalement un coût stable : le néerlandais VSNU 2019 est un amendement de l'accord de 2016, et se calque sur le montant de 2018 (même montant, même volume de publications). Les quatre dernières années du VSNU 2020 sont également stables. De la même manière, l'accord hongrois arbore un montant stable sur 3 ans, mais il s'agit d'une assiette globale sur toute la durée de l'accord, ensuite divisée par année. L'accord français est le seul où les montants décroissent sur 5 ans - un montant d'abonnement qui peut être complété par des frais d'APC. Le cas finlandais reste indéterminé car les sommes financières sont masquées dans l'accord. À l'exception de I'accord Couperin qui arbore des baisses effectives annuelles, les variations intra-accord sont globalement de deux types ${ }^{17}$ : soit il s'agit d'un montant fixe chaque année, soit on observe une augmentation liée à une "inflation » de 1,5\%, 2\%, 3\% ou 3,5\%. Nous insistons ici sur le fait qu'il s'agit bien d'une présentation, sans doute issue des négociations : on assume d'un côté l'idée d'un maintien des sommes, d'un « coût contrôlé " pour les consortiums, de l'autre une " inflation » justifiée par l'augmentation des volumes en accès ouvert ou le coût général des services de l'éditeur.

\footnotetext{
17 Les exceptions reposent sur des modifications importantes des services de l'éditeur ou du nombre d'institutions concernées par l'accord.
} 
L'aspect conventionnel peut avoir une importance cruciale dans la prochaine négociation, devant aboutir à la signature d'un nouvel accord. En effet, on sait que le point de départ sera presque toujours le montant de la dernière année de l'accord en cours. C'est le deuxième sens de l'évolution des montants, celle portant sur la période inter-accords. Toujours à partir de l'exemple d'Elsevier, des variations importantes sont manifestes. Pour les Pays-Bas, le dernier accord de 2020 constitue une hausse conséquente, avec un passage à de la publication illimitée en accès ouvert. Le cas de la France est complexe, puisque le montant d'un « Discount " n'est jamais totalement prévisible : les montants devraient évoluer à la baisse, sauf si les frais d'APC sont tels qu'ils rejoignent, voire dépassent, la somme payée en 2018. En revanche, la situation en Suède est très claire : le consortium Bibsam a commencé par passer à un montant nul en ne signant pas d'accord, avant de négocier une baisse de la somme totale tout en obtenant une publication en illimitée, avec une « inflation » plus faible que pour l'accord précédent.

Mais nous touchons là à une question tout autant épistémique que politique : que sont au juste les montants totaux? S'agit-il des sommes décaissées uniquement par les consortiums historiques, ou faut-il également inclure les APC payés directement par les chercheurs ou leurs institutions ? La Suède a clairement répondu à cette question en affichant sa « baisse » de montant : ce n'est qu'en prenant en compte ces montants parfois appelés « in the wild ", qu'on obtient une baisse effective, au lieu d'une augmentation du montant sur la base du seul contrat (figure 5-2).

Figure 5.2 - Evolution des montants payés par les institutions de Bibsam à Elsevier

\section{$€ 18,000,000$}

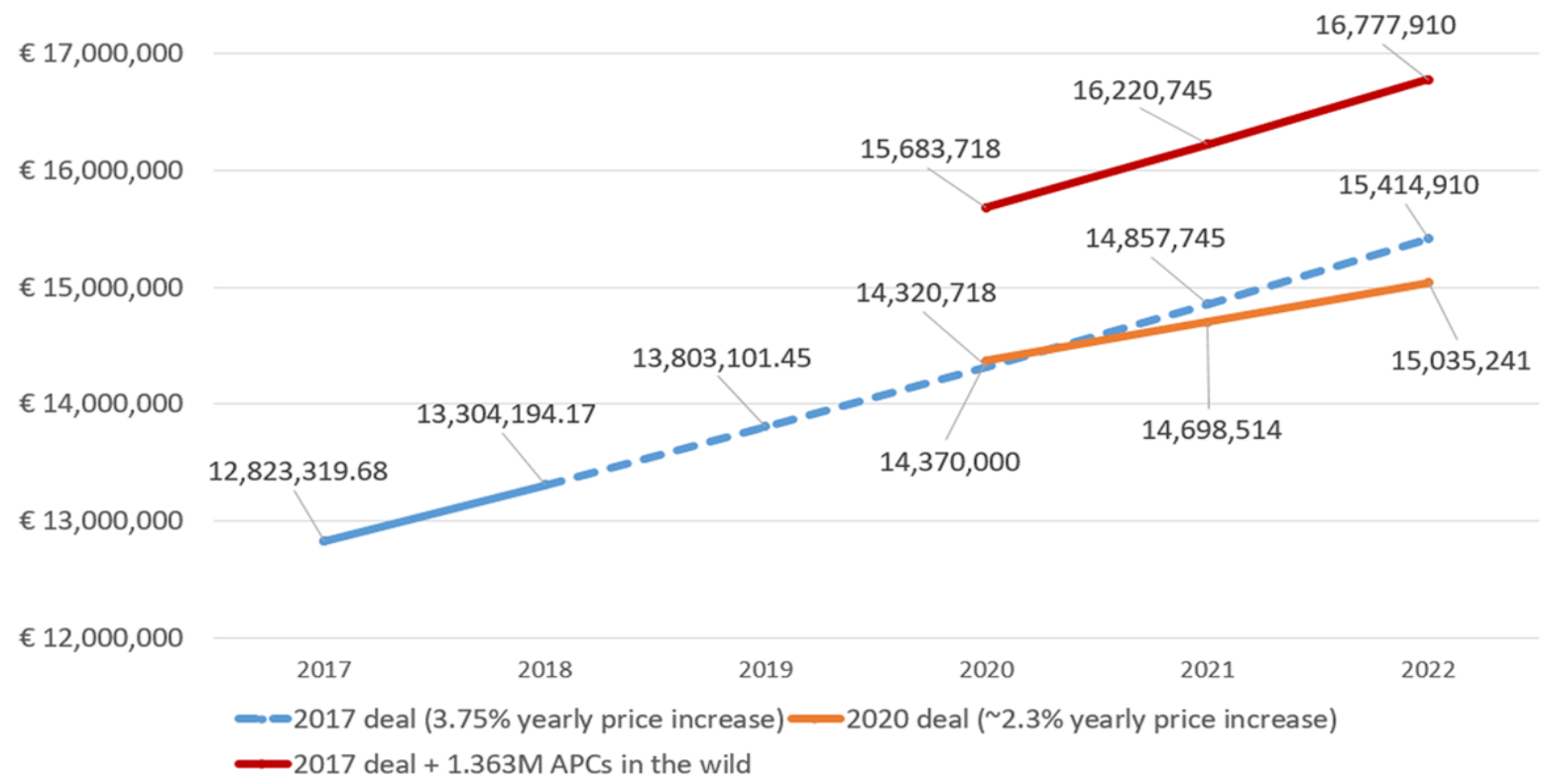

Source : Olsson, L., Lindelöw, C. H., Österlund, L., \& Jakobsson, F. (2020), Cancelling with the world's largest scholarly publisher: lessons from the Swedish experience of having no access to Elsevier. Insights, 33(1), 13. DOI: http://doi.org/10.1629/uksg.507 


\subsection{Répartition des sommes entre les payeurs}

Le montant associé aux accords pose une dernière question importante que nous n'avons pas encore abordée : celle de leur répartition entre les payeurs. Sur la base de notre corpus, on peut distinguer deux modèles principaux : un modèle de paiement centralisé (5.4.1) et un modèle multi-payeur (5.4.2).

\subsubsection{Le modèle centralisé}

L'écrasante majorité des accords (59 accords, $98 \%$ du total) se fonde sur un modèle de paiement centralisé : le consortium paie directement l'éditeur. Généralement, les versements sont réalisés en une fois ( 57 accords, $92 \%$ du total), avec un délai allant de 30 à 60 jours après la date à laquelle l'éditeur envoie sa facture. Plus rarement, des échéanciers s'articulent à des paiements échelonnés ( 5 accords, 8\% du corpus), à l'image du Hungary 2019 Wiley/EISZ qui paie l'éditeur deux fois par an au cours des trois années de l'accord. Dernière exception, unique celle-là, le cas du Netherlands 2016 Elsevier/VSNU-UKB, où des amendements nombreux ( 6 au total) mentionnent de nouveaux versements de la part du consortium, avec notamment l'ajout en cours d'accord de la collection Cell Press, en plus de la Complete Freedom Collection déjà souscrite.

Mais cette apparente simplicité laisse deux points aveugles concernant les accords qui arborent le modèle de paiement centralisé : premièrement, l'ignorance du montant total. Comme nous avons déjà eu l'occasion de le préciser, en dépit du caractère public de certains accords, les sommes ont été masquées (22 accords, 35\% du corpus). Deuxièmement, il s'agit de l'ignorance de la répartition des coûts au sein du consortium entre les institutions membres. En effet, pour la majorité des documents (57, soit $92 \%$ du total), nous ne savons rien du partage des coûts entre institutions, soit parce qu'il n'est pas mentionné, soit parce qu'il est masqué par des rectangles noirs. Seuls 5 accords ( $8 \%$ du total) donnent accès à la répartition interne des coûts (Norway 2019 Elsevier/UNIT; Sweden 2018 TandF/Bibsam; Sweden 2020 Sage/Bibsam, Sweden 2020 Wiley/Bibsam). Cependant, que les accords donnent à voir ou non la répartition interne des coûts, on ne connaît jamais les règles qui président à ladite répartition des coûts entre les institutions.

Face à cette ignorance relative, et après des échanges informels avec des membres des consortiums, ainsi que des présentations publiques mais non directement curables, on peut proposer deux modalités répandues de répartition. D'une part, une distribution reprenant le coût historique de l'abonnement, comme nous le savons de source informelle pour le consortium français Couperin. D'autre part, une répartition en fonction du volume de publication historique : plus une institution publie, plus elle paie. Entre la reprise de l'ancien modèle de "lecteur-payeur " et l'adoption de celui de "l'auteur-payeur" au niveau institutionnel, qui est aussi celui proposé par ACM Open pour assurer sa transition, toutes les formules intermédiaires sont évidemment envisageables. 


\subsubsection{Le modèle multi-payeur}

Le modèle multi-payeur est apparemment singulier à double titre. II n'est explicitement présent que dans un seul accord, à savoir, USA 2020 Springer $/$ UC $^{18}$, et cet accord a lui-même un statut particulier dans notre corpus, puisqu'il n'est pas encore véritablement signé. Le document dont nous disposons n'est pas juridiquement contraignant, c'est un "Memorandum of Understanding » qui anticipe une signature probable. Comme son nom l'indique, le modèle multi-payeur se distingue du modèle de paiement centralisé par I'intervention potentielle de plusieurs entités (en l'occurrence deux, University of California et l'auteur). Dans ce qui suit, nous proposons de détailler une partie du fonctionnement de la formule financière de USA 2020 Springer/UC. Nous verrons notamment les différents éléments sur lesquels l'accord propose une répartition entre University of California (UC) et l'auteur correspondant individuel.

Première répartition des frais, sur le paiement d'un article en accès ouvert (dans des revues hybrides uniquement). Les bibliothèques d'UC couvre les frais d'APC à hauteur de $1000 \$$. Les frais restants ("APC remainder ») sont soit payés par l'auteur correspondant luimême, soit par les bibliothèques d'UC lorsque durant le processus de publication, les auteurs précisent qu'ils ne disposent pas des fonds suffisants pour s'acquitter du reste de la somme due.

Deuxième répartition des frais, sur l'élaboration des bornes du Corridor de publications. Celles-ci sont calculées en plusieurs temps. On calcule d'abord un nombre d'articles de base (" base article number »), qui correspond au plus petit nombre d'articles publiés en accès ouvert en 2019 ou 2020. Ensuite, on calcule une borne basse, soit le « base article number » réduit de 15\%. Par ailleurs, on calcule une borne haute : 5\% au-dessus de la base en 2021, 10\% au-dessus de la base en 2022, 15\% au-dessus de la base en 2023. Dernière étape, on ajuste la borne haute en fonction du nombre d'auteurs qui, pour chaque année, est capable de payer sa part (" author participation »). En-dessous de 30\% de participation de l'auteur, la borne haute est réduite de $5 \%$. Entre $40 \%$ et $50 \%$ de participation de l'auteur, la borne est augmentée de $5 \%$; entre $50 \%$ et $60 \%$ de participation de l'auteur, la borne est encore augmentée de $5 \%$; au-delà de $60 \%$ de participation de l'auteur, la borne est encore augmentée de $5 \%$. Ainsi, la construction même du Corridor induit une répartition des frais entre UC et l'auteur, en prenant en compte la capacité d'autofinancement des auteurs.

Troisième répartition des frais, sur les règles de débordement du Corridor. Si le nombre d'articles publiés pour une année n'atteint pas le minimum, UC devra payer la différence (avec un APC individuel de $3208 \$$ ). Si le nombre d’articles publiés dépasse le maximum, les frais

\footnotetext{
18 II est également mobilisé dans l'accord USA 2020 UC/PLOS qui est un Pure Publish, et donc absent du corpus principal.
} 
d'APC de UC (les $1000 \$$ ou le total) seront supprimés, mais pas les frais de l'auteur («APC remainder $")$.

Quatrième répartition des frais, sur les sommes totales engagées dans l'accord. Pour les années 2021 et 2022, l'accord propose leur limitation : ainsi, en 2021, il ne peut dépasser 95\% du montant total de 2020, tandis que le montant de 2022 ne peut dépasser $98 \%$ du montant total de 2021. La limitation des frais passe par un remboursement de l'abonnement. Cela étant dit, l'accord prévoit une exception : si le montant total dépasse le montant de l'abonnement, le résidu ne sera pas remboursé, ce qui ouvre la voie à une facture illimitée pour UC.

Cette innovation de l'Université de Californie soulève plus généralement la question de l'articulation des paiements par les traditionnelles bibliothèques et celui provenant de fonds de recherche, notamment ceux alimentés par des financements contractuels ( « research grants »). À notre connaissance, les financeurs de la recherche ne participent pas directement à des accords, à une exception près. En effet, en Autriche, le FWF, équivalent de I'ANR français, est bien signataire, et prend en charge des articles à ses conditions (notamment une limitation du montant de l'APC). Ailleurs, tout en ayant montré leur soutien aux accords transformants comme moyen d'accélérer la transition vers l'accès ouvert, ils maintiennent des circuits de financement séparés, dédiés à la publication en accès ouvert. 


\section{Section 6.}

\section{Négocier la transition vers l'accès ouvert}

Au terme de l'exploration réalisée par cette étude sur les accords dits transformants, on peut retenir plusieurs éléments cruciaux en matière de description et d'analyse, afin de discuter, dans la conclusion, des briques nécessaires à toute recommandation en la matière. Comme le titre de cette section l'indique, les accords se présentent le plus souvent comme des tentatives de dépasser la simple reproduction d'une situation où trônent les abonnements, d'utiliser ces sommes données aux éditeurs pour faciliter la publication en accès ouvert. Dans ce qui suit, nous allons nous placer du point de vue des consortiums pour mettre en perspective ce que les accords nous apprennent sur la transition vers l'accès ouvert. Nous commencerons par insister sur le caractère singulier de chaque accord, fruit d'une négociation particulière (6.1). Au-delà de la spécificité de chaque accord, il est possible de repérer des grands " principes » qui guident la négociation des consortiums, en nous fondant sur l'exemple de ceux de Liber (6.2). Ensuite, nous discuterons du caractère expérimental de ces accords (6.3) avant d'aborder la question du caractère effectivement transformant des accords, qui fait l'objet d'une controverse grandissante (6.4).

\subsection{Une négociation singulière pour chaque accord}

Les accords sont le résultat plus ou moins refroidi de négociations particulièrement tendues et chaudes, dont on trouve la principale trace dans les commentaires suivant les " No Deal » comme ceux des University of California, du DEAL ou de Bibsam. La négociation elle-même ne nous est pas matériellement accessible et n'était pas l'objet central de notre étude, mais elle alimente clairement les résultats des accords finalement conclus. Premièrement, les conditions initiales de la négociation jouent un rôle central : le coût des abonnements actuels, les relations anciennes ou récentes entre consortium et éditeur, la place de ce dernier dans le paysage disciplinaire ou national constituent des aspects fondamentaux qui font une différence dès le départ des négociations. Cette dépendance aux conditions initiales, si 
diverses d'un pays à l'autre, voire d'une université à l'autre, joue un rôle crucial dans la diversité des montants engagés ${ }^{19}$.

Deuxièmement, les objectifs finaux des deux parties guident le processus de négociation et se matérialisent au moins partiellement dans l'accord tel qu'il existe, en matière de périmètre, d'inclusion de certaines dispositions, de coût total. On ne peut comprendre la très grande variété observée dans les parties précédentes en matière de morphologie et de mécanismes financiers des accords sans saisir le "contexte " de la négociation comme nous l'affirmait Ralf Schimmer (ancien " champion » de la cOAlition S, mais toujours directeur de l'information scientifique pour la Max Planck Digital Library) lors d'une visioconférence en septembre 2020. De notre point de vue, cela signifie qu'en dépit d'une certaine transparence des accords et d'un espace de circulation des négociateurs en voie de déconfinement - par le biais de la ligue des bibliothèques européennes de recherche (LIBER), du consortium SPARC ou d'échanges bilatéraux, on ne peut pas considérer le marché de l'accord transformant comme un espace unifié, ni même comme un espace dont l'évolution serait linéaire ou uniforme, mais plutôt comme le début de décloisonnement de "Big Deals » auparavant gérés en chambre de manière autonome, du moins du côté des consortiums.

\subsection{Des principes pour les négociations}

Outre le caractère spécifique de chaque accord, il est possible de repérer des grands principes qui sont censés guider l'action des consortiums lors de la signature d'accords. L'objectif de cette section est de comparer ces principes avec le contenu même des accords pour mesurer la permanence de leur mise en œuvre. Pour cela, prenons l'exemple des principes énoncés par LIBER, souvent cités par des consortiums dans leur communication (figure 6.1). Ces principes datent de 2017, donc avant la plupart des accords que nous avons étudiés, et sont en cours de redéfinition, comme l'atteste un récent workshop LIBER consacré à cette question, auquel nous avons assisté.

\footnotetext{
${ }^{19}$ Voir notamment le travail de révélation sur les montants d'abonnement au Royaume-Uni par l'usage des dispositions légales d'accès à l'information : Lawson, Stuart, and Ben Meghreblian. "Freedom of Information requests uncover the lack of transparency in journal subscription costs." Impact of Social Sciences Blog (2014).
} 

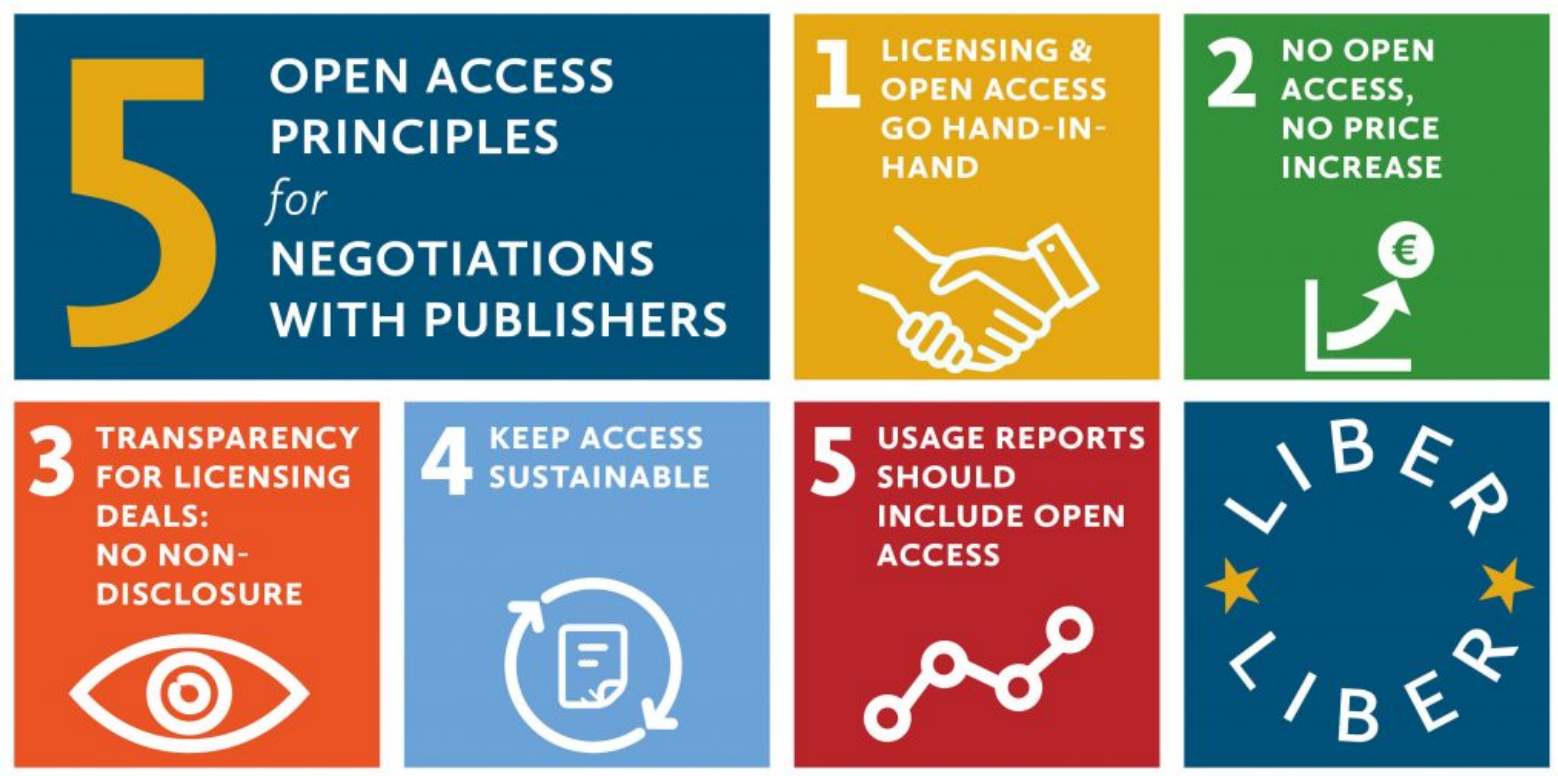

Source : https://ibereurope.eu/blog/2017/09/07/open-access-five-principles-for-negotiations-with-publishers/

Le premier principe de la figure 6.1 est celui de l'équipement juridico-technique des publications en accès ouvert par une licence dédiée. Sur la base de notre corpus de 62 accords analysés, mais aussi sur le corpus plus large, on peut affirmer que la mise en licence est désormais l'un des éléments communs des parties de publication en accès ouvert. De plus, les licences Creative Commons sont systématiquement indiquées dans le corps de l'accord : soit elles sont choisies en toute généralité (le plus souvent CC-BY), soit elles dépendent de la revue choisie pour la publication (CC-BY, CC-BY-NC, CC-BY-ND, CC-BY-NC-ND...).

Si ce principe est facilement vérifiable et effectivement rempli, ce n'est pas le cas du second, de nature financière, qui engage la question des augmentations, de la stabilité financière ( « cost neutrality »), voire plus récemment de la baisse des coûts par rapport aux accords précédents ${ }^{20}$. Nous avons souligné dans la section précédente toute la difficulté d'apprécier ces évolutions, en fonction de la base de départ (coût d'abonnement seul ou coût complet), de la prise en compte d'une inflation, du calcul des coûts de transaction et d'environnement. Dans les accords, il n'est pas fait référence à ce calcul de comparaison intertemporelle, mais les consortiums indiquent dans ESAC la tendance générale. En revanche, pour la durée de l'accord, nous avons vu que différents mécanismes pouvaient permettre de contrôler l'évolution du " coût total " pour le consortium, quand d'autres permettaient d'échapper à ces limites, que le coût soit assumé par le consortium, des établissements individuels ou les auteurs eux-mêmes.

${ }^{20}$ https://www.jisc.ac.uk/news/universities-will-cancel-deals-with-publishers-if-they-dont-respond-to-currentfinancial-pressures-13-aug-2020 
Le troisième principe a été en partie traité dans la section "méthodes " : la transparence comme principe se décline dans des formats différenciés d'ouverture du texte des accords, des dispositions financières, du détail des annexes, de la répartition du coût. La base ESAC a introduit la valeur d'un partage minimum, qui est cependant loin d'être adopté par tous les consortiums, et valorise un pragmatisme du as open as possible : quelques informations sur l'accord, la description de l'évolution générale des coûts, la mise à disposition partielle du texte de l'accord, l'ouverture complète des arrangements financiers. Il apparaît paradoxal que seuls quelques accords, eux-mêmes en tant que documents, soient dotés d'un DOI et d'une licence ouverte, alors même que ce statut est au cœur de l'objectif de publication. Comme nous l'avons vu, la culture du secret demeure un frein, que ce soit sous la forme directe du " secret des affaires » imposé par les éditeurs, ou l'absence de publicité du détail des dispositions contractuelles lorsque certaines parties à l'accord ne souhaitent pas devoir en répondre publiquement.

Le quatrième principe autour de la durabilité, vise tout particulièrement à sortir d'une logique de l'accès pur, entraînant une dépendance à l'éditeur et ses produits, en obligeant sans cesse à renouveler les accès. Si une majorité d'accords inclut des dispositions explicites de post-termination agreement ou un mécanisme d'achat de fonds patrimoniaux pour un usage sans limite, ce n'est pas le cas de tous. En revanche, du côté de la publication en accès ouvert, les licences CC garantissent une pérennité de l'accès électronique, sous réserve $d^{\prime}$ archivage et d'accès au réseau.

Enfin, le cinquième principe renvoie au travail d'équipement nécessaire, tant du côté de l'éditeur que du consortium, pour doter la relation de publication en accès ouvert des instruments adéquats de vérification et de suivi. Au comportement des lecteurs se substitue le comportement des auteurs (correspondants), leur inclinaison à entrer dans le dispositif de l'accord, et l'effet collectif de ces publications, mesurés par exemple par des baromètres de science ouverte.

Eu égard à notre corpus, les principes définis par LIBER apparaissent donc plutôt mis en œuvre par les consortiums, sous réserve d'une interprétation généreuse, notamment sur les arrangements financiers. Il faut également considérer que la plupart des consortiums envisagent ces accords comme un lieu d'expérimentation, dans lequel ces principes constituent davantage des guides que des conditions rigides nécessaires à une signature.

\subsection{La publication en accès ouvert comme expérimentation}

Si l'abonnement aux collections de revues est institutionnalisé depuis plus de 20 ans, le développement de la publication en accès ouvert dans un cadre orchestré par les consortiums est nettement plus récent. Minimisée dans les accords tournés vers l'abonnement, la publication en accès ouvert est régulièrement invoquée comme un horizon à atteindre dans 
les autres accords. Pour autant, l'incertitude, à la fois technique et financière, qui entoure son déploiement effectif s'accompagne souvent d'un statut singulier. Dans de nombreux accords, comme dans les communiqués de presse qui en font la promotion, la publication en accès ouvert revêt, explicitement ou non, un caractère expérimental. Plusieurs acceptions de ce statut peuvent cependant être distinguées.

La première tient dans la place limitée qu'occupe la publication dans l'accord, et plus précisément son statut d'exception par rapport au reste des clauses relevant d'un monde à accès limité. C'est tout particulièrement le cas de la mention de "pilote ", qui évoque bien un espace circonscrit au sein duquel il est possible de tester un objet d'un genre nouveau, aux caractéristiques peu stabilisées. Le caractère circonscrit dans le temps de la publication en accès ouvert constitue un autre élément notable : la délimitation temporelle des accords (entre 1 et 5 ans) ouvre momentanément l'espace des possibles. Autrement dit, à l'issue de l'accord, les éditeurs et les consortiums ont toujours la possibilité de revenir à un accord $\mathrm{d}^{\prime}$ abonnement traditionnel${ }^{21}$. La seule exception présente dans le corpus étudié sont les accords d'ACM open, dont les clauses stipulent une transition vers le "full open access » en sept ans.

Une deuxième acception possible de la notion d'expérimentation consiste à évoquer les risques anticipés autour de l'accès ouvert. La dimension risquée est repérable à plusieurs niveaux. D'abord, les accords ne durant que quelques années, ils n'engagent que très rarement les parties pour une négociation future. Ensuite, comme nous l'avons vu précisément dans la section sur les arrangements financiers, les incertitudes financières sont le plus souvent limitées et même lorsqu'il y a une prise de risque, celle-ci est encadrée comme dans l'accord allemand 2019 Springer/DEAL. Certains accords proposent de la publication en accès ouvert pour une partie des institutions seulement $-c^{\prime}$ est presque systématiquement le cas des accords néerlandais, qui distinguent les universités pouvant publier en accès ouvert, et celles de sciences appliquées, ou Hogescholen, exclues de cette offre. Enfin, des mécanismes de retrait («opt-out ») permettent d'abandonner la publication en accès ouvert afin de revenir à une formule classique d'abonnement (9 accords, 15\% du total). D'ailleurs, certains accords comme le UK 2019 Springer/JISC font explicitement le lien entre risque et capacité de sortie de l'accord.

"In recognition of the challenges associated with this new model and the need to monitor the agreement to ensure that it is sustainable, efficient, cost-effective and that it supports compliance, it has been agreed that it will be a $1+1+1$ agreement with optouts at the end of each year for both Springer and institutions. This is essential to mitigate risks ". (UK 2019 Springer/Jisc, p. 19).

\footnotetext{
${ }^{21}$ Un tel retour à une formule d'abonnement ne remettrait évidemment pas en cause l'ouverture des articles déjà publiés sur la période couverte par l'accord transformant. En revanche, les articles suivants cette période seraient de nouveau uniquement accessibles par un abonnement.
} 
Une troisième acception de la notion d'expérimentation tient dans le caractère généralement facultatif de la publication en accès ouvert. Alors que seulement $8 \%$ des accords du corpus imposent aux auteurs éligibles via l'éditeur la publication en accès ouvert, les $92 \%$ restant fonctionnent sur la base du volontariat. Ils n'imposent rien, même si l'accès ouvert est souvent présenté comme une option par défaut, que l'auteur peut abandonner au profit d'une publication sous abonnement. Par ailleurs, même dans les situations où la publication en accès ouvert est obligatoire, elle est suspendue à l'identification des auteurs comme éligibles, une opération qui n'est jamais garantie par avance: malgré cette incitation, les auteurs ont toujours la possibilité de ne pas donner les informations nécessaires à leur identification.

Le quatrième et dernier sens de la notion d'expérimentation appliquée à la publication en accès ouvert met l'accent sur ses effets encore mal connus. Les dispositifs de publication en accès ouvert présents dans les accords sont ici envisagés comme des objets exploratoires, sur lesquels il convient de produire des données permettant leur suivi et leur traçabilité, comme en témoignent les nombreux rapports que les éditeurs doivent systématiquement livrer aux consortiums et aux institutions. Et c'est sur cette base que le caractère transformant est aujourd'hui le plus souvent évalué.

\subsection{Des accords non transformants?}

En mars 2020, une coalition d'éditeurs « full $O A$ » a cosigné un " position paper » qui souligne le caractère inadapté de la terminologie " accord transformant " au vu du contenu réel des accords qu'elle désigne ${ }^{22}$. En effet, à leurs yeux, les dispositions des accords ne garantissent en rien une transition, notamment pour les raisons suivantes :

"They lack binding commitments to a full transformation to OA. Access is limited to selected parts of a publisher's portfolio. Conditions vary across national borders. They crowd-out pure OA publishers from institutional or national agreement negotiations $»$.

Notre étude confirme les trois premiers points, mais pas le dernier, puisque nous avons constaté la signature de nombreux accords avec des "full OA publishers " (Frontiers, MDPI, PLOS...) en 2019 et 2020. De plus, l'opposition entre éditeurs traditionnels signant des accords transformants et ces nouveaux acteurs n'est plus si tranchée : sur le plan capitalistique, Springer a depuis longtemps racheté BMC, Taylor \& Francis a récemment acquis F1000 ; en matière des accords, la stratégie du consortium suédois, Bibsam, l'a conduit à signer un accord " full $O A$ » avec Springer, dont le contenu pourrait tout aussi bien être celui d'un accord avec MDPI.

${ }^{22}$ https://frontiersinblog.files.wordpress.com/2020/03/position-statement-transformative-agreements.pdf 
Sans trancher sur les effets de lock-in du marché de la publication ou la prééminence des « big publishers » dans les budgets des consortiums, qui feraient l'objet d'une autre étude, nos résultats conduisent néanmoins à relativiser la portée transformante des accords signés pour plusieurs raisons combinées. II faut d'abord rappeler que deux définitions disjointes de la "transformation » cohabitent et, comme ici, se contredisent parfois. D'une part, la " transformation » porte directement sur le modèle économique des revues. L'ambition est de faire basculer les revues sous abonnement ou hybrides en revues en accès ouvert. C'est la perspective des financeurs de la recherche, et notamment celle de la Coalition S, qui souhaite transformer toute l'économie de la publication scientifique, en faisant de l'accès ouvert son principe de base. D'autre part, la "transformation" consiste essentiellement à modifier l'emploi des sommes payées par les consortiums aux éditeurs scientifiques, depuis l'abonnement vers la publication en accès ouvert. Pleinement transformer c'est ne plus payer pour l'abonnement, mais en faire un service additionnel de la publication en accès ouvert. Pour certains éditeurs, à l'image de Springer, cette deuxième définition convergerait naturellement vers la première : la réallocation des budgets de bibliothèques vers l'accès ouvert permettrait, à terme, de faire basculer l'économie des revues dans le monde de l'accès ouvert, en faisant du même coup disparaitre l'abonnement. C'est d'ailleurs dans cette optique que la cOAlition $S$ accepte de financer provisoirement les revues hybrides, du moment qu'elles font partie intégrante d'un accord transformant. Cependant, notre analyse nous invite plutôt à relativiser l'idée d'une convergence et à tenir clairement séparées les deux définitions : si les accords transformants, au sens de notre rapport, organisent bel et bien la redirection des budgets de bibliothèques vers la publication en accès ouvert, aucun dispositif contractuel ne pousse les revues à devenir des full OA (à l'exception du modèle de l'éditeur ACM Open). Le changement de modèle des revues n'a jamais été un objectif des consortiums, et c'est pour cela que cette définition est totalement absente des principes.

Même si l'on se focalise sur la deuxième définition, les clauses contractuelles empêchent mécaniquement d'évoluer vers une production entièrement en accès ouvert. Le choix systématique de limiter les articles éligibles aux auteurs correspondants des institutions, le caractère volontaire de la démarche dans la quasi-totalité des accords, le volume limité des articles publiables, le choix d'un périmètre restreint excluant notamment les revues supposément les plus prestigieuses (The Lancet, Cell, Nature...) sont autant de facteurs amenant à exclure de nombreux articles de l'accès ouvert.

De plus, en dépit de la croissance exponentielle du nombre d'accords, le volume total d'articles théoriquement « libérés " par la voie de ces dispositifs demeure extrêmement limité eu égard à une production mondiale de publications scientifiques de plusieurs millions d'articles par an. En effet, sur la base des déclarations ESAC, nous obtenons environ 85000 articles pour 2020 : en considérant que tous les accords n'y sont pas, mais seulement les plus importants en masse, nous pouvons estimer un nombre maximal de 120000 articles, soit moins de $5 \%$ de la production mondiale avec DOI. 
En l'absence de données immédiates et publiques sur la publication effective pour l'ensemble des accords, on peut opposer deux visions sur leur effet " libérateur » à partir d'exemples précis. La première vision met en doute la libération effective de la totalité des articles produits. À partir de l'exemple de l'accord Elsevier/SwissUniversities récemment signé, Christian Gurknecht a bâti des instruments de suivi de l'usage de l'accord qu'il a rendu publics $^{23}$. À la manière des Open Science Monitor ou du dispositif Open APC mis en place par OA2020, il donne ainsi à voir la réalité d'un accord non seulement très coûteux, mais qui ne remplit pas ses promesses. En effet, le volume d'articles publiés pour l'année 2020 (1814 articles) est bien inférieur à la borne maximale donnant lieu au calcul du coût total (2850 articles) et il met en évidence le nombre très important d'articles exclus de l'accord, du fait de leur format, de leur lieu de publication ou des auteurs non correspondant. Présentant ses premiers résultats ${ }^{24}$, il fait une critique argumentée de l'accord, concluant que seuls $30 \%$ de la production totale d'articles publiés par Elsevier sont pris en charge par l'accord, augmentant d'autant le prix individuel du «PAR fee ».

De manière contrastée, on peut considérer une seconde vision sur la base de la communication de Springer sur l'effet de ses accords signés en Europe. Sans fournir de données précises, l'éditeur affirme qu'ils assurent de fait un «flip » presque total pour la production nationale dans leurs revues:

" As the largest OA publisher of primary research, Springer Nature continues to drive forward the sustainable transition to OA for all authors regardless of discipline. Springer Nature now has 11 national TAs in place, the most out of any publisher. Recent data has shown that across the 8 countries with a national TA live in 2019, between $70 \%-90 \%$ of content [1] was published via gold OA (data takes into account the whole Springer Nature portfolio and therefore includes fully $O A$ content as well). Countries such as Sweden, Finland and Poland have, for example, seen $89 \%, 90 \%$ and $86 \%$ respectively, of Springer Nature content now being published gold OA since the introduction of their TAs [2]. The increase in $O A$ demonstrates that in countries where these arrangements are in place, a significant impact on the uptake of OA has occurred, far quicker than when relying on author uptake alone, and this has enabled the country to flip to achieve a near full OA environment for those authors publishing with Springer Nature $"{ }^{25}$

Si l'on comprend correctement les notes incluses dans cet extrait, c'est l'ensemble de la production nationale édité par le groupe d'édition - incluant donc BMC - qui est pris en compte, indépendamment de l'usage effectif des dispositions de l'accord. Ces ratios sont impressionnants, mais il faut les mettre en perspective sur une double base : d'une part, ce sont uniquement les auteurs correspondants qui sont pris en compte au numérateur et au

\footnotetext{
${ }^{23}$ https://oa-monitoring.ch/metabase/public/dashboard/a88453fb-aebc-4d90-b44f-ed37092f4dcf

${ }^{24}$ https://www.oa-monitoring.ch/post/the swiss 57 mio euro deal with elsevier/

${ }^{25} \mathrm{https}$ ://group.springernature.com/gp/group/media/press-releases/springer-nature-transformativeagreements/18311262
} 
dénominateur ; d'autre part, cela suppose une chaîne d'identification des institutions, dont on sait qu'elle est très complexe et qu'elle conduit sans doute à minorer le dénominateur du nombre total d'articles publiés.

La controverse sur la réalité et l'importance du « flip » réalisé par les accords courants devrait s'accentuer au fur et à mesure de la mise à disposition des données. Ainsi, le même Christian Gurknecht montre un volume bien plus important pour l'accord entre Elsevier et les institutions néerlandaises, pour un montant quasi-équivalent à l'accord suisse ${ }^{26}$. De plus, au vu de l'affichage communicationnel des accords récents, notamment les plus importants (Suède, Pays-Bas, Suisse, Allemagne), certains consortiums estiment avoir dépassé une phase pilote ou expérimentale et attendent donc de ces accords qu'une part significative de leur production soit mise à disposition par ce biais, avec des objectifs affichés de $100 \%$ de production pour les auteurs correspondants. En l'absence de véritables contraintes sur les auteurs et au vu des limites décrites ci-dessus, ce sont l'adhésion effective des auteurs à ce type de dispositif et la capacité des éditeurs à diriger ces flux de production qui détermineront son efficacité « libératoire ».

${ }^{26}$ https://oa-monitoring.ch/metabase/public/dashboard/92d3f93e-dcf1-4d9b-9156-6070bc80eac7 


\section{Section 7.}

\section{Quels objectifs pour les accords transformants?}

La section précédente a exposé les deux sens de la " transformation " attendue des accords. Si la version des financeurs est relativement facilement mesurable, celles des consortiums est plus complexe. Elle nécessite en amont la construction de bases de données permettant de connaître la production visée, sa part actuelle en accès ouvert, les coûts associés, etc ${ }^{27}$. Mais comme nous l'avons montré dans les sections de ce rapport, la place effective de l'accès ouvert dans l'accord n'est pas nécessairement prépondérante. II faut donc considérer deux dimensions importantes, que nous aborderons brièvement dans cette conclusion.

Premièrement, faire de l'accès ouvert un objectif ne passe pas nécessairement par ces accords. La situation états-unienne constitue un contre-exemple frappant : si les No Deal s'y multiplient avec les gros éditeurs, ce n'est généralement pas en raison de leur incapacité à produire un tel accord, mais parce que la volonté de frugalité des universités américaines est telle qu'elles préfèrent abandonner le Big Deal. Elles favorisent le retour à un abonnement simple, c'est-à-dire titre-à-titre, en s'appuyant sur les différentes versions en accès ouvert repérées par exemple par Unpaywall, des services tiers, et le prêt inter-bibliothèques pour fournir les articles désormais "inaccessibles " 28 . Ces pratiques d'unbundling sont collectionnées par SPARC, ainsi que les montants économisés ${ }^{29}$, dont une partie peut être utilisée soit pour financer la publication d'articles en accès ouvert par le paiement d'APC, soit pour soutenir des structures de publication en accès ouvert fondées sur un modèle Diamant ${ }^{30}$.

Deuxièmement, la négociation en vue d'accords transformants peut avoir d'autres objectifs que la transition vers l'accès ouvert. En prenant l'exemple des futures négociations entre Couperin et Springer, nous avons rappelé dans le tableau 7.1 ci-après qu'une telle négociation s'inscrit dans des politiques plus générales, qu'elles soient liées à la science

\footnotetext{
${ }^{27}$ Voir Olsson, L., Lindelöw, C.H., Österlund, L. and Jakobsson, F., 2020. Cancelling with the world's largest scholarly publisher: lessons from the Swedish experience of having no access to Elsevier. Insights, 33(1), p.13.

${ }^{28}$ https://scholarlykitchen.sspnet.org/2020/04/13/bundle-question/

${ }^{29}$ Voir la liste non exhaustive sur le site de SPARC https://sparcopen.org/our-work/big-deal-cancellationtracking/

${ }^{30}$ Le « modèle diamant » est un modèle économique de revue scientifique, dont la caractéristique est de publier des articles en accès ouvers sans faire payer ni le lecteur ni l'auteur. Il est généralement opposé au modèle de l'auteur-payeur, également appelé « Gold-APC ».
} 
ouverte (archivage institutionnel, refus des APC, soutien à des structures de publication non commerciales, principe de bibliodiversité...) ou à d'autres logiques budgétaires et institutionnelles. Loin d'être la voie unique ou la dernière étape d'une histoire évolutionniste, nous avons montré que les accords, dans leur diversité, matérialisent provisoirement des objectifs, une trajectoire, des besoins et des budgets. Leur durée limitée et leur caractère réversible constituent des éléments intrinsèques de leur nature contractuelle. 
Tableau 7.1 - Exemple de six scénarios

\begin{tabular}{|c|c|c|c|c|c|c|c|c|}
\hline Scénario & Principe directeur & $\begin{array}{c}\text { Type } \\
\text { d'accord }\end{array}$ & $\begin{array}{c}\text { Forme d'accès } \\
\text { ouvert }\end{array}$ & $\begin{array}{l}\text { Périmètre } \\
\text { des revues }\end{array}$ & $\begin{array}{l}\text { Publier } \\
\text { en OA } \\
\text { requis }\end{array}$ & $\begin{array}{l}\text { Arrangement } \\
\text { financier }\end{array}$ & $\begin{array}{l}\text { Ordre de grandeur } \\
\text { des montants }\end{array}$ & Exemples \\
\hline 1 & $\begin{array}{l}\text { Basculer la } \\
\text { production sous } \\
\text { abonnement en } \\
\text { AO }\end{array}$ & $\begin{array}{l}\text { Read \& } \\
\text { Publish }\end{array}$ & $\begin{array}{l}\text { Publication limitée } \\
\text { via un fonds d'APC }\end{array}$ & $\begin{array}{l}\text { Hybrides } \\
\text { seulement }\end{array}$ & oui & $\begin{array}{l}\text { Montant } \\
\text { variable }\end{array}$ & $\begin{array}{l}\text { Norvège: } 2.200 .000 € \\
\text { Suède: } 4.100 .000 €\end{array}$ & $\begin{array}{c}\text { Norvège (Springer-Unit 2020- } \\
\text { 2022) } \\
\text { Suède (Springer-Bibsam } \\
\text { 2019-2021) }\end{array}$ \\
\hline 2 & $\begin{array}{l}\text { Répartition des } \\
\text { coûts } \\
\text { et des risques }\end{array}$ & $\begin{array}{l}\text { Publish \& } \\
\text { Read }\end{array}$ & Publication illimitée & $\begin{array}{l}\text { Hybrides } \\
\text { seulement }\end{array}$ & non & $\begin{array}{l}\text { Montant } \\
\text { variable mais } \\
\text { encadré }\end{array}$ & $\begin{array}{c}26.612 .500 € \text { sur une } \\
\text { base de } 2750 € / \text { article. }\end{array}$ & $\begin{array}{c}\text { Allemagne (Springer-Projekt } \\
\text { Deal 2020-2022) }\end{array}$ \\
\hline 3 & $\begin{array}{l}\text { Payer un coût fixe } \\
\text { pour l'ensemble } \\
\text { de la publication } \\
\text { en } A O\end{array}$ & $\begin{array}{l}\text { Publish \& } \\
\text { Read }\end{array}$ & Publication illimitée & $\begin{array}{l}\text { Hybrides et } \\
\text { full OA } \\
\text { journals }\end{array}$ & oui & Montant fixé & $\begin{array}{c}\text { Suède: } 1.440 .000 € \\
\text { Pays-Bas: } \\
\text { 16.000.000€ }\end{array}$ & $\begin{array}{c}\text { Suède (Elsevier-Bibsam } \\
\text { 2020-2022) } \\
\text { Pays-Bas (Elsevier-VSNU- } \\
\text { UKB 2020-2024) }\end{array}$ \\
\hline 4 & $\begin{array}{l}\text { Faire de l'AO un } \\
\text { service annexe }\end{array}$ & $\begin{array}{l}\text { Read \& } \\
\text { Discount }\end{array}$ & $\begin{array}{c}\text { Discount sur la } \\
\text { publication en OA + } \\
\text { une archive ouverte } \\
\text { sous } 12 \text { et } 24 \text { mois }\end{array}$ & $\begin{array}{l}\text { Hybrides et } \\
\text { full OA } \\
\text { journals }\end{array}$ & non & $\begin{array}{l}\text { Montant } \\
\text { variable }\end{array}$ & $? ?$ & $\begin{array}{l}\text { France (Elsevier-Couperin } \\
\text { 2019-2022) }\end{array}$ \\
\hline 5 & $\begin{array}{l}\text { Status quo et } \\
\text { évitement } \\
\text { d'un lock-in }\end{array}$ & Subscription & / & $\begin{array}{l}\text { Revues } \\
\text { sous } \\
\text { abonnement }\end{array}$ & / & Montant fixé & $? ?$ & $\begin{array}{l}\text { France (Springer-Couperin } \\
\text { 2019) }\end{array}$ \\
\hline 6 & $\begin{array}{l}\text { Respect des } \\
\text { principes }\end{array}$ & No Deal & / & / & / & / & / & $\begin{array}{l}\text { Allemagne (Elsevier-Projekt } \\
\text { Deal) depuis juillet } 2018\end{array}$ \\
\hline
\end{tabular}




\section{Annexe : présentations des travaux}

\section{Mai 2020}

Présentation des avancées de l'étude au Secrétariat Permanent pour la Science Ouverte (SPSO)

\section{8 août 2020}

Communication au congrès 4S/EASST initialement prévu à Prague dans la session 198 intitulée "Transformations and tensions in academic publishing". Titre de la présentation: « Contracting in the open access age. Unboxing 'Big Deals' in academic publishing ».

Disponible ici $:$ https://convention2.allacademic.com/one/ssss/ssss20/index.php?cmd=Onlin e+Program+View+Paper\&selected paper $i d=1655312 \&$ PHPSESSID $=$ siheeib1h5i7cmp035h 7k $\underline{\mathrm{dnmrm}}$

\section{7 octobre 2020}

Présentation des premiers résultats de l'étude au SPSO.

\section{6 octobre 2020}

Communication au $2^{\text {nd }}$ Basel Sustainable Publishing Forum - The Global Transition to Open Access: Challenges and Solutions, initialement prévu à Bâle. Titre de la présentation: "Transformative Agreements to Achieve Open Access - a First Systematic Analysis of Available Consortium-Publisher Documents ".

Vidéo archivée ici : https://www.youtube.com/watch?v=VdTng1dAq1s\&feature=emb logo

\section{4 novembre 2020}

Invitation à communiquer au congrès de I'International Coalition of Library Consortium (ICOLC), initialement prévu à Thessalonique. Titre de la présentation: "Transformative Agreements to Achieve Open Access - a First Systematic Analysis of Available ConsortiumPublisher Documents ». 